\author{
Universidade de São Paulo \\ Instituto de Física
}

\title{
Emaranhamento de feixes de fótons por meio do campo magnético
}

\author{
Ricardo Alexander Castro Pinto
}

Orientador: Prof. Dr. Dmitri Maximovitch Guitman

Tese de doutorado apresentada ao Instituto de Física para obtenção do título de Doutor em Ciências

Banca Examinadora:

Prof. Dr. Dmitri Maximovitch Guitman (IFUSP)

Prof. Dr. Arnaldo Gammal (IFUSP)

Prof. Dr. Josif Frenkel (IFUSP)

Prof. Dr. Dmitry Vasilevich (UFABC)

Prof. Dr. Galen Mihaylov Sotkov (UFES) 


\section{FICHA CATALOGRÁFICA \\ Preparada pelo Serviço de Biblioteca e Informação do Instituto de Física da Universidade de São Paulo}

Castro Pinto, Ricardo Alexander

Emaranhamento de feixes de fótons por meio do campo magnético. São Paulo, 2016.

Tese (Doutorado) - Universidade de São Paulo. Instituto de Física. Depto. de Física Nuclear

Orientador: Prof. Dr. Dmitri Maximovitch Guitman

Área de Concentração: Física

Unitermos: 1. Emaranhamento quântico; 2. Informação quântica; 3. Mecânica quântica; 4. Campo magnético. 
Dedico este trabajo a mi padre Ricardo y a mi abuelo Teodoro. 


\section{Agradecimentos}

Ao Prof. Dmitri Maximovitch Gitman, pela dedicação constante à minha orientação, pelo relacionamento de amizade, pelo exemplo de dedicação ao trabalho e pela ajuda imprescindível no desenvolvimento e edição deste manuscrito.

Ao Prof. Alexandre Levine pela amizade e ajuda imprescindível no desenvolvimento da tese.

Ao meu amigo Igor Viktorevich Tyutin, pelos diversos exemplos e sabedoria recebidos durante todo o período da sua visita em São Paulo.

Ao Prof. Iuda Dawid Goldman Vel Lejbman, pelo relacionamento de amizade, exemplo constante de dedicação ao trabalho e pelo apoio imprescindível durante o período do doutorado.

Ao Dr. João Geraldo Beggiato, quem ama verdadeiramente a física, pela amizade, constantes aulas de humor e apoio recebido no período do doutorado.

Ao João Luis Meloni Assirati pela amizade, constantes aulas de humor, português e pela ajuda imprescindível no período do doutorado.

Ao Pavel Moshin pela amizade e colaboração.

À todos os professores visitantes, em particular, Boris Voronov, Anatoly Shabad, Peter Lavrov, Dmitry Vasilevich, Vladimir Man'ko, Margarita Man'ko, Alexei Deriglazov, Alexei Semikhatov, Vladislav Bagrov, Serguei Gavrilov, pela amizade e colaboração durante o período das visitas ao grupo Quanta.

Aos professores Galen Mihaylov Sotkov, Josif Frenkel e Arnaldo Gammal, membros da banca examinadora.

Aos membros da banca do exame de qualificação pelos comentários, observações e conselhos para a realização do manuscrito.

Ao Instituto de Física da USP e seus funcionários, em particular, Éber Pato e Cláudia Barioni pela disponibilidade, apoio e paciência.

Ao $\mathrm{CNPq}$ pelo suporte financeiro. 


\section{Resumo}

Foi proposta uma experiência na qual seria possível produzir um emaranhamento quântico de feixes de fótons com diferentes frequências, movendo-se em uma mesma direção, controlado por meio de um campo magnético externo.

Nessa experiência, a interação entre o campo magnético e fótons é realizada por intermédio de elétrons, que interagem tanto com os fótons quanto com o campo magnético externo. Foi desenvolvida uma teoria que descreve processos físicos. Derivamos medidas de emaranhamento de informação e de Schmidt para um sistema geral de dois qubits e a medida residual para um sistema geral de três qubits. Usando a informação obtida da análise dos sistemas de dois e de três quase-fótons, calculamos medidas de emaranhamento.

Criamos um programa para cálculo numérico, nesses casos, através do qual construímos gráficos de dependência das medidas de emaranhamentos em feixes de dois e de três fótons. Os resultados obtidos nos permitem ver como a medida de emaranhamento depende dos parâmetros, que caracterizam o sistema em questão. Por exemplo, se ambas as polarizações dos fótons coincidem, então, nenhum emaranhamento ocorre. O emaranhamento acontece apenas se as polarizações do fóton forem opostas. 


\begin{abstract}
An experiment is proposed which can produce a quantum entanglement of photon beams having different frequencies and moving in the same direction. The experiment is controlled by an external magnetic field. In this experiment, the interaction between the photons and magnetic field is carried out by means of electrons interacting both with the photons and the external magnetic field. A theory is developed which describes physical processes in this experiment. The meausre of entanglement of information and the measure of Schmidt are calculated for the general system of two qubits, as well as the residual measure for general system of three qubits. Using the information obtained from the analysis of the systems of two and three quasi-photons, we have calculated the entanglement measures in such cases. A computer program is created for numerical calculations in such cases which enables one to construct the graphs of dependence for entanglements of measures in bundles of two and three photons. The results allow us to see how the entanglement measure depends on the parameters that characterize the system in question. For example, if both polarizations of the photons coincide, then no entanglement takes place. The entanglement occurs only if the polarization of the photon are opposite.
\end{abstract}




\section{Lista de Figuras}

4.1 Medida de informação $E_{I}\left(\Psi_{\gamma}(\downarrow, \uparrow)\right)$ como uma função de $\omega$ e $\Delta \kappa$, para $\epsilon=0.1 \ldots \ldots \ldots \ldots \ldots \ldots$

4.2 Medida de Schmidt $E_{S}\left(\Psi_{\gamma}(\downarrow, \uparrow)\right)$ como uma função de $\omega$ e $\Delta \kappa$, para $\epsilon=0.1 \ldots \ldots \ldots \ldots \ldots$. . . . . . . . . . 56 


\section{Sumário}

1 Introdução $\quad 9$

2 Feixes de fótons interagindo com elétrons no campo magnético 14

2.1 Equação de Dirac com onda plana quantizada . . . . . . . . . 14

2.2 O movimento na ausência de um campo externo . . . . . . . . 20

2.3 Feixes de fótons interagindo com elétrons no campo magnético 24

3 Emaranhamento em sistemas quânticos compostos $\quad 29$

3.1 Descrição dos estados quânticos dos sistemas físicos . . . . . . 30

3.2 Sistemas quânticos compostos do numero finito de qubits . . . 34

3.3 Estados separáveis e emaranhados . . . . . . . . . . . . . 37

3.4 Sistemas de dois qubits . . . . . . . . . . . . . . . . 37

3.5 Sistemas de três qubits . . . . . . . . . . . . . . . . . . . . . . . . 42

3.6 Medidas do emaranhamento . . . . . . . . . . . . . . . 45

3.6.1 Testemunhas de emaranhamento . . . . . . . . 45

3.6.2 Medida de emaranhamento . . . . . . . . . . . . 46

3.6.3 O cálculo de emaranhamento de sistema de dois qubits em caso geral . . . . . . . . . . . . . . . . 47

3.6.4 O emaranhamento do estado puro de três qubits em caso geral . . . . . . . . . . . . . . . . . 49

4 Emaranhamento em feixes de fótons com frequências diferentes $\quad 51$

4.1 Emaranhamento em dois feixes de fótons . . . . . . . . . . 51

4.1.1 Fótons com polarizações antiparalelas . . . . . . . . . 51

4.1.2 Fótons com polarizações paralelas alinhadas ao longo do campo magnético . . . . . . . . . . . . . 55 
4.2 Emaranhamento em três feixes de fótons . . . . . . . . . . 57

5 Conclusões $\quad 62$

$\begin{array}{ll}\text { A Notações } & 70\end{array}$

B Anexos $\quad 73$

B.1 Programa para cálculo numérico em Matlab . . . . . . . . . 73

B.2 Caso de dois fótons no Mathematica . . . . . . . . . . . 79

B.3 Caso de três fótons no Mathematica . . . . . . . . . . . . . . 81 


\section{Capítulo 1}

\section{Introdução}

Em 1935 Albert Einstein, juntamente com Boris Podolsky e Nathan Rosen, escreveu um artigo intitulado "Can quantum mechanical description of physical reality be considered complete?" [1], no qual eles descrevem um experimento mental ("Gedankenexperiment", como denominado originalmente em alemão), que ficou conhecido como o paradoxo de Einstein-Podolsky-Rosen (EPR). Sumariamente, o paradoxo EPR consiste em uma tentativa de indicar a incompletude da mecânica quântica com a ajuda de experimentos, como a medição indireta de parâmetros de um micro objeto sem exercer, sobre o mesmo, uma influência direta. O objetivo dessa medida indireta é extrair mais informações sobre o estado dos micro-objetos do que a descrição da mecânica quântica permite.

Como é bem conhecido, o paradoxo EPR fundamenta-se em princípios básicos das leis de conservação da mecânica clássica e em suas implicações em mecânica quântica. O exemplo clássico se refere ao caso de duas partículas idênticas, $A$ e $B$, que surgem do colapso de uma terceira partícula $C$. De acordo com a lei da conservação do momento, a soma do momento de $A$ e de $B$ deve ser igual ao momento de $C$, isto é, $\mathbf{p}_{A}+\mathbf{p}_{B}=\mathbf{p}_{C}$.

Esta simples lei de conservação implica na possibilidade de se medir, indiretamente, o momento de $B$ através de uma medição direta do momento de $A$, sem, contudo, perturbar o movimento de $B$.

Por outro lado, suponhamos que exista um aparato físico capaz de medir as coordenadas de $B$, de modo que se obtenha, como fruto do resultado dessas medidas, a coordenada e o momento de $B$, com precisão e simultaneamente. Entretanto, de acordo com o princípio de Heisenberg, coordenada e momento são autovalores de operadores incompatíveis e, portanto, não podem 
ser medidos simultaneamente com precisão. Logo, conclui-se que a relação de incerteza não é absoluta e as leis da mecânica quântica são incompletas.

De acordo com N. Bohr, esse experimento mental não contradiz a interpretação probabilística da mecânica quântica de Kopenhagen. De fato, Einstein, em contraste com a interpretação de Kopenhagen, implicitamente, acreditava na existência de variáveis escondidas em mecânica quântica.

Sob o ponto de vista da interpretação de Kopenhagen, a explicação é simples: No experimento de EPR, a medição do momento da primeira partícula cria uma redução da função de onda total, de modo que o estado da segunda partícula torna-se um estado com um impulso definitivo. A medida das coordenadas da segunda partícula afeta o seu momento, de acordo com as regras usuais da mecânica quântica. Logo, concluir que ocorreu medição simultânea da coordenada e do momento não faz sentido sob essa interpretação.

O experimento de EPR é incomum, em termos da física clássica. O estado da segunda partícula muda perante uma medida efetuada sobre a primeira partícula, independentemente da distância entre elas. A função de onda total do sistema é uma grandeza não-local, sendo, portanto, descrita como um estado quântico emaranhado.

A ideia de variáveis escondidas foi explicitamente desenvolvida posteriormente por D. Bohm [2] e o paradoxo de EPR foi modificado de modo a considerar duas partículas de spin $1 / 2$, ou dois fótons com frequências distintas e polarizações arbitrárias. Em sua construção, Bohm supôs uma correlação não-trivial entre as partículas $A$ e $B$, e introduziu os chamados estados quânticos emaranhados. Dessa forma, Bohm reconsiderou o experimento de EPR com estado emaranhado como um estado final de partículas não-interagentes $A$ e $B$.

Em 1964, Bell deduziu as chamadas desigualdades de Bell, que são válidas em uma teoria com variáveis escondidas [3]. Através de experimentos envolvendo a desigualdade de Bell, pode-se testar a existência de algumas consequências do fenômeno de emaranhamento em mecânica quântica, impossíveis de acordo com uma imagem clássica do mundo, caracterizada pela noção de realismo local.

Os estados emaranhados também foram amplamente utilizados nessas desigualdades. Atualmente, deve-se notar que a violação das desigualdades de Bell é registrada em vários experimentos diferentes, veja, por exemplo, [4]. Consequentemente, não existem, até o momento, critérios estabelecidos que confirmem uma teoria de variáveis escondidas. Desse modo, os estados emaranhados tornaram-se uma ferramenta poderosa no estudo de questões 
principais em mecânica quântica como, por exemplo, na teoria da informação e computação quântica $[3,5,6,7]$. As mais úteis e interessantes aplicações dos estados emaranhados são a codificação superdensa [46], teletransporte quântico [45] e criptografia quântica [8].

Acreditamos que a compreensão completa subjacente ao emaranhamento quântico ainda exige considerações detalhadas de uma variedade de casos relativamente simples, não somente em mecânica quântica não-relativística, mas também em teoria quântica de campos (TCQ). Isso explica o recente interesse em se estudar emaranhamento quântico e entropia de estados quânticos em TCQ para sistemas com vácuo instável, em particular, com campos de fundo fortes que podem criar partículas do vácuo, veja, por exemplo, [18, 19, 20, 21, 22, 23, 24]. Nesses casos, modelos com soluções exatas podem ser muito úteis.

Nesta área, uma das tarefas mais importantes e interessantes consiste em como preparar estados emaranhados com uma determinada medida de emaranhamento. Portanto, nesta tese de doutorado, estudamos a possibilidade de emaranhamento de dois e três feixes de fótons em interação com um campo magnético e apresentamos uma possibilidade de realizar este processo.

Além de puro interesse acadêmico, o efeito controlado de emaranhamento quântico é um tópico atualmente estudado devido a possíveis aplicações em teoria da codificação. A princípio, acredita-se que o emaranhamento quântico controlado pode servir como um meio de transmissão de sinais codificados, veja, por exemplo $[5,6,7]$.

Abordamos, na tese, a criação de emaranhamento quântico controlado por feixes de fótons com diferentes frequências, movendo-se em uma mesma direção e com a ajuda de um campo magnético externo. Mais especificamente, a interação entre o campo magnético e fótons é realizada por intermédio de elétrons que interagem tanto com os fótons quanto com o campo magnético externo (na aproximação linear, o campo eletromagnético - feixes de fótons - não interage com outro campo eletromagnético - campo magnético externo).

Para tanto, utilizamos soluções exatas de um problema de mecânica quântica relativística a fim de apresentarmos maneiras de se afetar a medida de emaranhamento de um sistema composto por fótons, que se move em uma mesma direção, com frequências distintas e com quaisquer possíveis polarizações lineares, sendo que cada um interage com um campo magnético externo aplicado.

O mecanismo referente a esse problema é ilustrado da seguinte forma: 
Seja I uma determinada área do espaço onde um feixe de fótons é criado e emitido para uma região II, onde há elétrons não-interagentes, porém, sob a influência de um campo magnético $\mathbf{B}$. O feixe de fótons, ao passar pela região II, é direcionado para uma região III, onde há um detector de fótons. Dessa forma, fótons originados em I e detectados em III atravessam uma região II, que interage com um campo magnético por intermédio de elétrons não-interagentes. Esse processo é ilustrado conforme a Figura 1, a seguir:

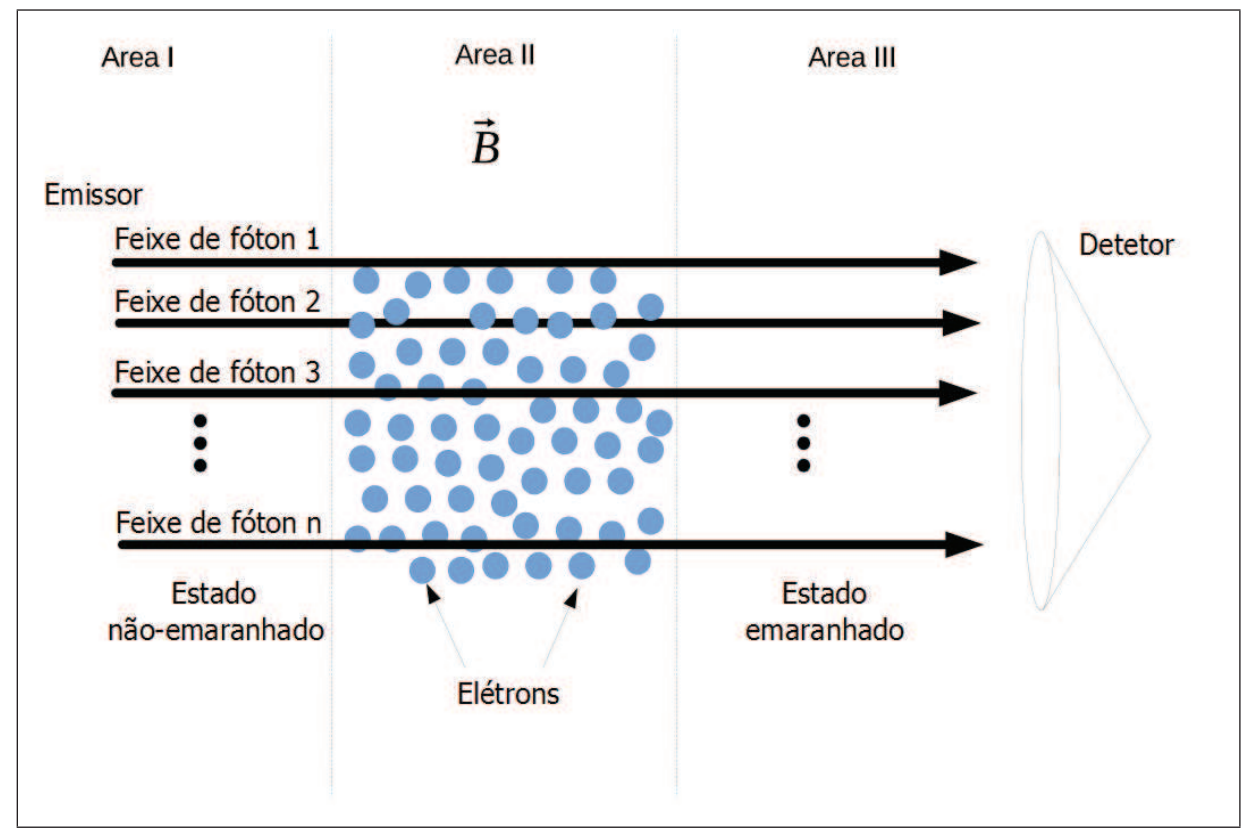

Figura 1. Esquema proposto para emaranhamento de feixes de fótons por meio do campo magnético.

No experimento proposto, por exemplo, um dispositivo emissor gera, em um instante de tempo inicial, dois fótons que são descritos por um estado quântico não-emaranhado e, ao mesmo tempo, um campo magnético é aplicado em elétrons não-interagentes em II. Ao atravessarem a região II, os fótons são detectados pelo receptor, em III, que analisa se o estado quântico desses fótons é emaranhado. A medida do emaranhamento dependerá do campo magnético aplicado de modo que a análise da mesma é a decodificação do sinal.

A descrição teórica desse experimento consiste em calcular a dependência do emaranhamento sob o campo magnético aplicado. Para isso, estudamos 
as soluções exatas das equações quânticas de movimento, que descrevem a interação de um elétron (ou um meio de elétrons não-interagentes) com um campo quantizado de vários fótons - que se movem em uma mesma direção, com diferentes frequências e polarizações lineares arbitrárias.

Além disso, em um campo magnético externo apropriado, submetemos o sistema do elétron que interage com o campo do fóton quântico. Demonstramos que as equações quânticas de movimento, referidas anteriormente, podem ser deduzidas a partir de um problema mais amplo, a saber, o do elétron em interação com uma onda plana quantizada, como apresentado nas referências. [25, 26, 27, 28, 29].

A equação em questão é uma equação de Dirac modificada com potenciais eletromagnéticos, operadores quânticos, que atuam em variáveis dos fótons, argumentos da função de onda. Essa dedução é apresentada no Capítulo 2 em que analisamos e resolvemos exatamente a equação de Dirac modificada.

Os estados quânticos do feixe de vários fótons interagindo com elétrons podem ser representados como estados de dois subsistemas, sendo que um deles representa um sistema de quase-fótons livres, cujas frequências diferem das frequências iniciais dos fótons. Estudamos soluções especiais das equações quânticas que descrevem os dois e três quase-fótons. De acordo com o esquema proposto de observação do emaranhamento controlado, acreditamos que o observador detectará feixes de quase-fótons não-emaranhados.

Analisando-os como estados de fótons livres, eles, então, representam estados emaranhados. De fato, analisando esse estado em termos de fótons livres, o receptor irá obter a medida do emaranhamento. Tecnicamente, o emaranhamento é descrito pelo coeficiente de decomposição do estado quântico não-emaranhado de quase-fótons em todos os estados possíveis de fótons livres.

No Capítulo 3, apresentamos uma descrição de emaranhamento quântico específica para esse problema, mostramos os critérios e a medida do emaranhamento para sistemas quânticos de dois e três "qubits". No Capítulo 4, calculamos emaranhamentos de um estado puro, de forma geral, de dois e três fótons com frequências diferentes e polarizações arbitrárias. A fórmula geral obtida e os resultados do Capitulo 2 são utilizados em cálculos numéricos de emaranhamento controlado de dois e três fótons em um campo magnético. 


\section{Capítulo 2}

\section{Feixes de fótons interagindo com elétrons no campo magnético}

Nesta seção, estudamos os estados quânticos de feixes de fótons interagindo com um elétron. Para este fim, considera-se a equação de Dirac modificada em que os potenciais eletromagnéticos são a soma de duas partes $A_{\mu}(x) \mathrm{e}$ $\hat{A}_{\mu}(x)$. Aqui, $A_{\mu}(x)$ são potenciais eletromagnéticos clássicos de um campo externo e $\hat{A}_{\mu}(x)$ são operadores de potenciais eletromagnéticos de um campo de onda plana. A função de onda bi-espinor de tal equação de Dirac depende tanto das coordenadas $x$ do elétron quanto das variáveis dos fótons. Os operadores $\hat{A}_{\mu}(x)$ agem nessas últimas variáveis. No que segue, chamamos tal equação modificada de equação de Dirac com onda plana quantizada. Estudamos as soluções exatas de tal equação modificada especialmente no caso em que o campo eletromagnético externo é um campo magnético uniforme constante e os estados quânticos do campo eletromagnético podem ser interpretados como os estados de quase-fótons.

\subsection{Equação de Dirac com onda plana quan- tizada}

A seguir, apresentamos uma interpretação física da equação de Dirac com onda plana quantizada no contexto da QED [34, 35]. 
Seja $\hat{H}_{\text {QED }}$ o Hamiltoniano da eletrodinâmica quântica (QED), $|\Psi\rangle$ um vetor de estado da interação de Dirac $\psi(x)$ e $A^{\mu}(x)$ os campos eletromagnéticos (campos de elétron-pósitron e fótons). Na representação de Schrödinger, os vetores de estado dependem do tempo $t=x^{0}$, de modo que $|\Psi\rangle=\left|\Psi\left(x^{0}\right)\right\rangle$, e a evolução temporal de $\left|\Psi\left(x^{0}\right)\right\rangle$ é descrita pela equação de Schrödinger (usamos unidades onde $c=\hbar=1$ )

$$
i \frac{\partial\left|\boldsymbol{\Psi}\left(x^{0}\right)\right\rangle}{\partial x^{0}}=\hat{H}_{\mathrm{QED}}\left|\boldsymbol{\Psi}\left(x^{0}\right)\right\rangle .
$$

O espaço total de estados da QED pode ser considerado como o produto direto do espaço de Hilbert da partícula e o espaço de Fock dos estados de fótons. Sabe-se que a base Ket no subespaço elétron-pósitron pode ser tomada da seguinte forma:

$$
\langle 0| \psi\left(\mathbf{r}_{1}\right) \ldots \psi\left(\mathbf{r}_{n}\right) \bar{\psi}\left(\mathbf{r}_{1}^{\prime}\right) \ldots \bar{\psi}\left(\mathbf{r}_{m}^{\prime}\right)
$$

(veja, por exemplo, Ref. [36]). Nessa equação, $\langle 0|$ é o vetor de vácuo no subespaço elétron-pósitron e $\psi(\mathbf{r}), \bar{\psi}(\mathbf{r})$ são operadores do campo de Dirac na representação de Schrödinger. Vamos considerar as seguintes amplitudes de probabilidade:

$$
\left\langle 0\left|\psi\left(\mathbf{r}_{1}\right) \ldots \psi\left(\mathbf{r}_{n}\right) \bar{\psi}\left(\mathbf{r}_{1}^{\prime}\right) \ldots \bar{\psi}\left(\mathbf{r}_{m}^{\prime}\right)\right| \boldsymbol{\Psi}\left(x^{0}\right)\right\rangle .
$$

Por um lado, essas amplitudes são vetores no espaço de Fock de estados de fótons e, por outro lado, eles são $4^{n+m}$ funções de onda componente dos estados com um determinado número de partículas carregadas, elétrons e pósitrons. É óbvio que o conjunto infinito de amplitudes (2.2) é equivalente ao vetor de estado $\left|\Psi\left(x^{0}\right)\right\rangle$. Em particular, a primeira amplitude,

$$
\left\langle 0|\psi(\mathbf{r})| \Psi\left(x^{0}\right)\right\rangle=\Psi(x), x=\left(x^{0}, \mathbf{r}\right),
$$

é a projeção do vetor $\left|\Psi\left(x^{0}\right)\right\rangle$ sobre o estado de um único elétron e pode ser considerada como uma função de onda de quatro componentes de um elétron interagindo com os fótons.

A equação (2.1) induz um conjunto de equações acopladas para amplitudes (2.2). Se o campo eletromagnético é quantificado no gauge de Coulomb, onde, em particular, $\hat{A}_{0}=0$, e $\operatorname{div} \hat{\mathbf{A}}(\mathbf{r})=0$, veja e.g. [37, 38, 36, 39], então a equação correspondente escrita para a amplitude de um único elétron (2.3) tem a forma

$$
i \frac{\partial \boldsymbol{\Psi}(x)}{\partial x^{0}}=\hat{H}_{e, \gamma} \boldsymbol{\Psi}(x)+\Delta,
$$


onde

$$
\hat{H}_{e, \gamma}=\hat{H}_{\gamma}+\boldsymbol{\alpha} \hat{\mathbf{P}}+m \beta, \hat{\mathbf{P}}=\hat{\mathbf{p}}-e \hat{\mathbf{A}}(\mathbf{r}) .
$$

Nesse caso, $\hat{H}_{\gamma}$ é o hamiltoniano de fótons transversais livres, $\hat{\mathbf{A}}(\mathbf{r})$ é o operador potencial vetorial de tais fótons na representação de Schrödinger, e todas as contribuições das amplitudes de muitas partículas (2.2) são absorvidas no termo $\Delta$. Os operadores $\hat{H}_{\gamma}$ e $\hat{\mathbf{A}}(\mathbf{r})$ podem ser representados como combinações de operadores de criação e aniquilação de fótons $a_{\mathbf{k}, \lambda}^{+}$e $a_{\mathbf{k}, \lambda} \mathrm{com}$ momentos definidos $\mathbf{k}$ e polarizações $\lambda=1,2$, que obedecem às relações de comutação de Bose

$$
\begin{aligned}
& a_{\mathbf{k}, \lambda} a_{\mathbf{k}^{\prime}, \lambda^{\prime}}^{+}-a_{\mathbf{k}^{\prime}, \lambda^{\prime}}^{+} a_{\mathbf{k}, \lambda}=\delta_{\mathbf{k}, \mathbf{k}^{\prime}} \delta_{\lambda, \lambda^{\prime}}, \\
& a_{\mathbf{k}, \lambda} a_{\mathbf{k}^{\prime}, \lambda^{\prime}}-a_{\mathbf{k}^{\prime}, \lambda^{\prime}} a_{\mathbf{k}, \lambda}=a_{\mathbf{k}, \lambda}^{+} a_{\mathbf{k}^{\prime}, \lambda^{\prime}}^{+}-a_{\mathbf{k}^{\prime}, \lambda^{\prime}}^{+} a_{\mathbf{k}, \lambda}^{+}=0 .
\end{aligned}
$$

Assim,

$$
\begin{aligned}
& \hat{H}_{\gamma}=\sum_{\mathbf{k}, \lambda}|\mathbf{k}| a_{\mathbf{k}, \lambda}^{+} a_{\mathbf{k}, \lambda} \\
& \hat{\mathbf{A}}(\mathbf{r})=\sum_{\mathbf{k}, \lambda} \frac{1}{\sqrt{2|\mathbf{k}| V}}\left[a_{\mathbf{k}, \lambda} e^{i(\mathbf{k x})} \mathbf{e}_{\lambda}(\mathbf{k})+a_{\mathbf{k}, \lambda}^{+} e^{-i(\mathbf{k x})} \mathbf{e}_{\lambda}^{*}(\mathbf{k})\right]
\end{aligned}
$$

Aqui, $V=L^{3}$ é o volume da caixa de quantização e $\mathbf{e}_{\lambda}(\mathbf{k})$ são vetores de polarização que possuem as propriedades

$$
\mathbf{e}_{\lambda}(\mathbf{k}) \mathbf{k}=\mathbf{e}_{\lambda}^{*}(\mathbf{k}) \mathbf{k}=0, \mathbf{e}_{\lambda}^{*}(\mathbf{k}) \mathbf{e}_{\lambda^{\prime}}(\mathbf{k})=\delta_{\lambda, \lambda^{\prime}}, \lambda, \lambda^{\prime}=1,2 .
$$

Se o potencial (2.7) é considerado como sendo, por exemplo, espacialmente periódico com o período $L$ ao longo de cada eixo cartesiano, então, o vetor $\mathbf{k}$ tem os seguintes valores discretos:

$$
\mathbf{k}=2 \pi L^{-1}\left(n_{1}, n_{2}, n_{3}\right), n_{s} \in \mathbb{Z} .
$$

O operador $\hat{H}_{e, \gamma}$ pode ser considerado como um Hamiltoniano de uma carga $e$ interagindo com o campo eletromagnético quantizado (fóton).

Ignorando os efeitos da criação de pares virtuais e a aparição relacionada das amplitudes mais altas na eq. (2.4) (i.e., desprezando o termo $\Delta$ ), nos resta a equação de Schrödinger para uma única carga $e$ interagindo com o campo de fótons transversal. Esta equação é a base para muitos modelos do tipo de um único elétron. A amplitude (2.3) pode ser tratada como uma 
função de onda de um elétron interagindo com o campo de fóton transversal. Formalmente é um bi-espinor, cujas componentes residem no espaço de Fock dos fótons transversais. No que segue, chamamos isso de bi-espinor de Fock.

Soluções da equação de Schrödinger com o potencial (2.7) e $\Delta=0$ tornam possível estudar, na forma de modelo (i.e., sem considerar a criação real e virtual de pares de elétrons-pósitrons), os problemas em que uma única carga é envolvida juntamente com um número arbitrário de fótons. Desafortunadamente, mesmo com esta simplificação, o problema ainda não tem todas as soluções exatas, de modo que somos forçados a tratar a interação radiativa como uma perturbação, começando com as soluções da equação livre como aproximação inicial não perturbada. É, no entanto, bastante evidente que existam algumas situações para as quais correções para a solução do problema livre não são pequenas. Por exemplo, se os estados com uma grande densidade de fótons $\rho$ forem abordados, deve-se levar em conta todos os termos da expansão de perturbação, uma vez que os elementos da matriz perturbativos são proporcionais a $e^{2} \rho$.

Vamos considerar a possibilidade de tomar em conta a série de perturbação toda. Suponha que estamos interessados em processos onde um elétron em um campo de onda plana intenso está envolvido. Do ponto de vista da mecânica quântica, isso significa que não surgem estados iniciais com uma grande densidade de fótons que se movem numa direção comum $\mathbf{n}$ ao longo da onda plana. Então, é razoável escolher a expressão (2.5) para o Hamiltoniano não perturbado na equação (2.4), em que ÂA é a parte do potencial do campo eletromagnético quantizado contendo apenas a somatória dos fótons transversais que se movem na direção comum $\mathbf{n}$ (os fótons da onda plana). Se esse problema pode ser resolvido exatamente, então, a interação de uma carga com os fótons da onda plana é mantida exatamente, ao passo que as correções para uma solução desse tipo poderiam ser pequenas.

A seguir, nesta Seção, consideramos a equação de Schrödinger da forma

$$
i \frac{\partial \Psi(x)}{\partial x^{0}}=\hat{H}_{e, \gamma} \boldsymbol{\Psi}(x), \quad \hat{H}_{e, \gamma^{\mathrm{pw}}}=\hat{H}_{\gamma^{\mathrm{pw}}}+\boldsymbol{\alpha} \hat{\mathbf{P}}+m \beta, \hat{\mathbf{P}}=\hat{\mathbf{p}}-e \hat{\mathbf{A}}(\mathbf{r})
$$

onde $\hat{H}_{e, \gamma^{\mathrm{pw}}}$ é o Hamiltoniano de fótons transversais livres que estão se movendo na direção comum $\mathbf{n}$ (a Hamiltoniana do campo de onda plana livre) e $\hat{\mathbf{A}}(\mathbf{r})$ é o correspondente operador de potencial vetorial desses fótons. Se $\mathbf{n}$ for escolhido ao longo do eixo $z, \mathbf{n}=(0,0,1)$, temos para os fótons que 
estão se movendo na direção positiva de $z$,

$$
\begin{aligned}
& \mathbf{k}=2 \pi L^{-1}(0,0, s)=\kappa_{s} \mathbf{n}, \quad \kappa_{s}=\kappa_{0} s, \quad \kappa_{0}=2 \pi L^{-1}, s \in \mathbb{N}, \\
& a_{s, \lambda}=a_{\mathbf{k}, \lambda}, \quad a_{s, \lambda}^{+}=a_{\mathbf{k}, \lambda}^{+} .
\end{aligned}
$$

Logo,

$$
\begin{aligned}
& \hat{H}_{\gamma^{\mathrm{pw}}}=\sum_{s \in \mathbb{N}, \lambda} \kappa_{s} a_{s, \lambda}^{+} a_{s, \lambda} \\
& \hat{\mathbf{A}}(\mathbf{r})=\sum_{s \in \mathbb{N}, \lambda} \frac{1}{\sqrt{2 \kappa_{s} V}}\left[a_{s, \lambda} e^{i \kappa_{s}(\mathbf{n x})} \mathbf{e}_{\lambda}(s)+a_{s, \lambda}^{+} e^{-i \kappa_{s}(\mathbf{n x})} \mathbf{e}_{\lambda}^{*}(s)\right] .
\end{aligned}
$$

Ao passar para a representação de interação com relação ao Hamiltoniano $\hat{H}_{\gamma^{\mathrm{pw}}}$,

$$
\Psi(x)=\exp \left(-i \hat{H}_{\gamma^{\mathrm{pw}}} x^{0}\right) \Psi(x),
$$

na equação (2.10), podemos descobrir que o bi-espinor de Fock $\Psi(x)$ satisfaz a equação de Dirac

$$
\left(\gamma^{\mu} \hat{P}_{\mu}-m\right) \Psi(x)=0, \quad \hat{P}_{\mu}=i \partial_{\mu}-e \hat{A}^{\mu}(x)
$$

com o operador de potencial eletromagnético

$$
\hat{A}^{\mu}(x)=\left(0, \exp \left(i \hat{H}_{\gamma^{\mathrm{pw}}} x^{0}\right) \hat{\mathbf{A}}(\mathbf{r}) \exp \left(-i \hat{H}_{\gamma^{\mathrm{pw}}} x^{0}\right)\right) .
$$

Este operador de potencial eletromagnético tem a forma

$$
\hat{A}^{\mu}(x)=\hat{A}^{\mu}\left(u_{0}\right)=\sum_{s \in \mathbb{N}, \lambda} \frac{1}{|e|} \sqrt{\frac{\delta}{\kappa_{s}}}\left[a_{s, \lambda} e^{-i \kappa_{s} u_{0}} e_{\lambda}^{\mu}(s)+a_{s, \lambda}^{+} e^{i \kappa_{s} u_{0}} e_{\lambda}^{\mu *}(s)\right] .
$$

Aqui, $\delta=e^{2} / 2 L^{3}$, considerando que a variável $u_{0}$ é apenas o cone de luz temporal $u_{0}=n x$ que toma a forma $u_{0}=x^{0}-x^{3}$ quando $\mathbf{n}$ é escolhido ao longo do eixo $x$, i.e., $\mathbf{n}=(0,0,1)$. Os vetores de polarização estão agora rotulados por números inteiros $s$ e pelo $\lambda$, e obedecem as condições

$$
\begin{aligned}
& \left.e_{\lambda}^{\mu}(s)=\left(0, \mathbf{e}_{\lambda}(\mathbf{k})\right)\right), \quad \mathbf{k}=\kappa_{s} \mathbf{n}, \\
& n e_{\lambda}(s)=n e_{\lambda}^{*}(s)=0, e_{\lambda}^{*}(s) e_{\lambda^{\prime}}(s)=-\delta_{\lambda, \lambda^{\prime}}, n^{\mu}=(1, \mathbf{n}), n^{2}=0 .
\end{aligned}
$$


Os operadores de criação e aniquilação de fótons $a_{s, \lambda}^{+}$e $a_{s, \lambda}$ estão agora rotulados por inteiros $s$ e pelo $\lambda$ de acordo com as equações (2.11) e obedecem às relações de comutação tipo Bose

$$
\begin{aligned}
& a_{s^{\prime}, \lambda^{\prime}} a_{s, \lambda}^{+}-a_{s, \lambda}^{+} a_{s^{\prime}, \lambda^{\prime}}=\delta_{s, s^{\prime}} \delta_{\lambda, \lambda^{\prime}}, \\
& a_{s^{\prime}, \lambda^{\prime}} a_{s, \lambda}-a_{s, \lambda} a_{s^{\prime}, \lambda^{\prime}}=a_{s^{\prime}, \lambda^{\prime}}^{+} a_{s, \lambda}^{+}-a_{s, \lambda}^{+} a_{s^{\prime}, \lambda^{\prime}}^{+}=0 .
\end{aligned}
$$

A quantidade $\delta$ na equação (2.15) caracteriza a força da interação entre a carga e o campo de ondas planas, se interpretarmos $V^{-1}=L^{-3}$ como a densidade de elétrons $\rho$ na região em que o campo de fótons interage com elétrons. Então $\delta \sim e^{2} \rho$. A dimensionalidade de $\delta$ é $[\delta]=l^{-3}$ (onde $l$ é a dimensionalidade do comprimento). Sendo escrita com $\hbar$ e $c$ restabelecidos, tem a forma:

$$
\delta=\frac{e^{2}}{\hbar c} \frac{1}{2 L^{3}}=\frac{\alpha \rho}{2} .
$$

onde $\alpha=e^{2} / \hbar c=1 / 137$ é a constante de estrutura fina.

Finalmente, se um campo eletromagnético externo clássico $A_{\mu}^{\text {ext }}(x)$ atua sobre o sistema em consideração (elétrons interagindo com a onda plana quantizada), seus potenciais devem ser combinados com o potencial (2.15) de uma maneira aditiva, de modo que a equação para o bi-espinor de Fock $\Psi(x)$ tenha a forma da equação de Dirac com os potenciais compostos de dois termos

$$
A^{\mu}=\hat{A}^{\mu}(x)+A_{\text {ext }}^{\mu}(x) .
$$

Vamos supor que o potencial de campo externo clássico que é uma combinação dos campos transversais e longitudinais. Evidentemente, para tal superposição, os campos magnéticos e elétricos estão relacionados como

$$
\mathbf{H}=[\mathbf{n} \times \mathbf{E}]+(\mathbf{n H}) \mathbf{n} \Longrightarrow \mathbf{E}=-[\mathbf{n} \times \mathbf{H}]+(\mathbf{n E}) \mathbf{n} .
$$

Os campos submetidos à relação (2.20) constituem a classe geral dos campos para os quais a integração da equação de Dirac pode ser realizada.

Logicamente, toda a combinação (2.19) fornece o campo (já operador) da mesma estrutura (2.20). Esta escolha do campo clássica é devida ao fato de que só para campos desta estrutura é possível separar as variáveis de spin seguindo o método padrão e, como foi demonstrado em Ref. [40], apenas esses campos tornam a maior separação de variáveis possível. 
Podemos separar as variáveis de spin em eq. (2.14) representando o biespinor de Fock da forma

$$
\begin{aligned}
& \Psi(x)=\mathcal{K}\left\{[1+(\boldsymbol{\sigma} \mathbf{n})] \psi_{1}(x)+[1-(\boldsymbol{\sigma} \mathbf{n})] \psi_{-1}(x)\right\} v \\
& \mathcal{K}=\left(\begin{array}{c}
m+(n \hat{P})+(\boldsymbol{\sigma} \mathbf{n})\left(\boldsymbol{\sigma} \hat{\mathbf{P}}_{\perp}\right) \\
{[m-(n \hat{P})](\boldsymbol{\sigma} \mathbf{n})+\left(\boldsymbol{\sigma} \hat{\mathbf{P}}_{\perp}\right)}
\end{array}\right), \hat{\mathbf{P}}_{\perp}=\hat{\mathbf{P}}-\mathbf{n}(\boldsymbol{\sigma} \hat{\mathbf{P}}) .
\end{aligned}
$$

O bi-espinor $v$ é constante, enquanto que as funções $\psi_{\zeta}(\zeta= \pm 1)$ satisfazem as equações independentes

$$
\begin{aligned}
& \left(\hat{P}_{0}^{2}-\hat{\mathbf{P}}^{2}-m^{2}+i e E_{3}-\zeta e \bar{H}_{3}\right) \psi_{\zeta}(x)=0, \\
& E_{3}=(\mathbf{n E}), \quad H_{3}=(\mathbf{n H}) .
\end{aligned}
$$

\subsection{O movimento na ausência de um campo externo}

Se estivermos lidando somente com partículas em movimento na onda plana quantizada somente (sem qualquer campo externo), podemos definir $A_{\text {ext }}^{\mu}=0$ em eq. (2.19). Então, o bi-espinor de Fock pode ser escrito como

$$
\Psi(x)=\mathcal{K} \Phi(x) v,
$$

onde $\Phi(x)$ é uma solução da equação Klein-Gordon correspondente (pode ser chamado de Fock-scalar),

$$
\left(\hat{P}^{2}-m^{2}\right) \Phi(x)=0 .
$$

Observamos facilmente que, neste caso, a quantidade $(n \hat{P})=(n \hat{p})$ é uma integral de movimento. Consideraremos que o bi-espinor de Fock seja um autovetor do operador $(n \hat{p})$ com o autovalor igual a $(n p)$. Definindo

$$
\begin{aligned}
& \Phi(x)=\exp \left[-\frac{i}{2}(n p) u_{3}\right] \varphi\left(u_{0}, \mathbf{r}_{\perp}\right), \\
& u_{3}=(\bar{n} x), \quad \bar{n}^{\mu}=(1,-\mathbf{n}), \quad \mathbf{r}_{\perp}=\mathbf{r}-\mathbf{n}(\mathbf{n r}),
\end{aligned}
$$

obtemos, nesse caso, que a função $\varphi\left(u_{0}, \mathbf{r}_{\perp}\right)$ satisfaz a equação de Schrödinger:

$$
i \partial_{0} \varphi\left(u_{0}, \mathbf{r}_{\perp}\right)=\mathcal{H} \varphi\left(u_{0}, \mathbf{r}_{\perp}\right), \quad \mathcal{H}=\frac{\hat{\mathbf{P}}_{\perp}^{2}+m^{2}}{2(n p)}
$$


Considere os operadores

$$
G_{\mu}=i \partial_{\mu}+n_{\mu} \sum_{s \in \mathbb{N}, \lambda} \kappa_{s} a_{s, \lambda}^{+} a_{s, \lambda}
$$

que comutam entre si $\left[G_{\nu}, G_{\mu}\right]_{-}=0$, e com todos os operadores $\hat{P}_{\mu},\left[\hat{P}_{\mu}, G_{\nu}\right]_{-}=$ 0 . É também evidente, em virtude da propriedade $n^{2}=0$ que $(n G)=(n \hat{p})$. Pode-se ver que o bi-espinor de Fock $\Psi$ pode ser escolhido para serem autofunções dos operadores $G_{\mu}$,

$$
G_{\mu} \Psi=g_{\mu} \Psi .
$$

Vemos que, a partir de (2.28), que as diferenciações das coordenadas $i \partial_{\mu}$ podem ser eliminadas da eq. (2.26). Assim, ficamos com equações que contêm apenas as variáveis de fótons. O operador $G_{\mu}$ possui, evidentemente, o significado do operador de energia-momento para o sistema composto da partícula e da onda plana quantizada.

Para a equação de Dirac, é conveniente escrever o operador $G_{\mu}$ como

$$
\begin{aligned}
& G^{\mu}=p_{D}^{\mu}+n^{\mu} \mathcal{H}_{D}, \quad p_{D}^{\mu}=p_{K}^{\mu}+R_{0} n^{\mu}, \\
& \mathcal{H}_{D}=\mathcal{H}_{K}-R_{0}, \quad R_{0}=\frac{e \sigma^{\alpha \beta} F_{\alpha \beta}}{4(n g)}, \\
& F_{\alpha \beta}=\partial_{\alpha} A_{\beta}-\partial_{\beta} A_{\alpha}=n_{\alpha} A_{\beta}^{\prime}-n_{\beta} A_{\alpha}^{\prime},
\end{aligned}
$$

onde o primeiro denota a derivada em relação a $u_{0}$. Nessa equação, $p_{D}^{\mu}$ e $\mathcal{H}_{D}$ são integrais de movimento.

Poderia parecer que, para separar as variáveis de partículas e de fótons, é necessário que as eqs. (2.28) sejam cumpridas. Porém, este não é o caso. Vamos transformar o bi-espinor de Fock ou o escalar de Fock usando o operador unitário

$$
U_{1}=\exp \left(i u_{0} \sum_{s \in \mathbb{N}, \lambda} \kappa_{s} a_{s, \lambda}^{+} a_{s, \lambda}\right) .
$$

As seguintes relações são facilmente obtidas

$$
\begin{aligned}
& U_{1}^{+} G_{\mu} U_{1}=i \partial_{\mu}, \quad U_{1}^{+} \hat{P}_{\mu} U_{1}=i \partial_{\mu}+Q_{\mu}-n_{\mu} \sum_{s \in \mathbb{N}, \lambda} \kappa_{s} a_{s, \lambda}^{+} a_{s, \lambda}, \\
& Q^{\mu}=(0, \mathbf{Q}), \quad \mathbf{Q}=\sum_{k \in \mathbb{N}, \lambda} \sqrt{\frac{\delta}{\kappa_{k}}}\left[a_{k, \lambda} \mathbf{e}_{\lambda}(k)+a_{k, \lambda}^{+} \mathbf{e}_{\lambda}^{*}(k)\right] .
\end{aligned}
$$


com a ajuda delas, finalmente concluímos que os bi-espinores de Fock podem ser escritas como

$$
\begin{aligned}
& \Psi=\exp \left[-\frac{i}{2}(n p) u_{3}\right] U_{1} \overline{\mathcal{K}} \psi_{0} v, \\
& \overline{\mathcal{K}}=\left(\begin{array}{c}
m+(n p)+(\boldsymbol{\sigma} \mathbf{n})(\boldsymbol{\sigma} \mathbf{F}) \\
{[m-(n p)](\boldsymbol{\sigma} \mathbf{n})+(\boldsymbol{\sigma} \mathbf{F})}
\end{array}\right), \mathbf{F}=-i \boldsymbol{\nabla}_{\perp}+\mathbf{Q}, \\
& \nabla_{\perp}=\boldsymbol{\nabla}-\mathbf{n}(\mathbf{n} \boldsymbol{\nabla}),
\end{aligned}
$$

onde a função $\psi_{0}$ é uma solução da equação de Schrödinger

$$
i \partial_{0} \psi_{0}=\mathcal{H} \psi_{0}, \quad \mathcal{H}=\frac{\mathbf{F}^{2}+m^{2}}{2(n p)}+\sum_{s \in \mathbb{N}, \lambda} \kappa_{s} a_{s, \lambda}^{+} a_{s, \lambda} .
$$

Observe a validade das seguintes relações

$$
\begin{aligned}
\mathcal{H} & =U_{2}^{-1} U_{1}^{+} \mathcal{H}_{D} U_{1} U_{2}, \\
U_{2} & =1-\frac{\left(\gamma^{\mu} n_{\mu}\right)\left(\gamma^{\nu} Q_{\nu}\right)}{2(n p)}, \quad U_{2}^{-1}=1+\frac{\left(\gamma^{\mu} n_{\mu}\right)\left(\gamma^{\nu} Q_{\nu}\right)}{2(n p)} \\
\hat{p}^{\mu} & =U_{2}^{-1} U_{1}^{+} p_{D}^{\mu} U_{1} U_{2}^{-1}=i \partial^{\mu}-n^{\mu} \mathcal{H}
\end{aligned}
$$

Agora, estamos em condições de separar as variáveis de partículas. Isso corresponde a escolher as funções $\Psi$ como autofunções dos operadores $p_{D}^{\mu}$ com os autovalores $p^{\mu}$ (os autovalores $p^{\mu}$ são, de acordo com (2.34), os mesmos para ambos os operadores $\left.p_{D}^{\mu}\right)$. Logo, escrevemos, em vez de (2.32),

$$
\Psi=\exp [-i(p x)] U_{1} \overline{\mathcal{K}} \psi v,
$$

onde o operador $\overline{\mathcal{K}}$ é definido pela eq. (2.32) em que deve-se estabelecer

$$
\mathbf{F}=\mathbf{p}_{\perp}+\mathbf{Q}, \quad \mathbf{p}_{\perp}=\mathbf{p}-\mathbf{n}(\mathbf{n p}),
$$

e a função $\psi$ é a solução da equação de Schrödinger

$$
i \partial_{0} \psi=\hat{H} \psi, \quad \hat{H}=\sum_{s \in \mathbb{N}, \lambda} \kappa_{s} a_{s, \lambda}^{+} a_{s, \lambda}+\frac{(\mathbf{p Q})}{(n p)}+\frac{\mathbf{Q}^{2}}{2(n p)} .
$$

O momento-energia da quase-partícula satisfaz a condição natural

$$
p^{2}=m^{2} \Longrightarrow p_{0}^{2}=\mathbf{p}^{2}+m^{2} .
$$


Finalmente, podemos, agora, escrever as soluções, satisfazendo eqs. (2.28)

$$
\Psi=\exp [-i(g x)] U_{1} \overline{\mathcal{K}} \psi_{R} v,
$$

onde $\overline{\mathcal{K}}$ é definida em (2.32) sob a condição (2.36), e $\psi_{R}$ depende das variáveis de fótons por si só e é uma solução da equação de Schrödinger estacionária

$$
\hat{H} \psi_{R}=R \psi_{R} .
$$

$\mathrm{O}$ vetor momento-energia $g^{\mu}$ do sistema possui a forma

$$
g^{\mu}=p^{\mu}+n^{\mu} R \text {. }
$$

É a soma do vetor de momento-energia $p^{\mu}$ da quase-partícula e a soma dos quase-fótons, $R n^{\mu}$.

Dessa maneira, se a soma é efetuada ao longo das duas possíveis polarizações $\lambda$ para cada $s$ fixo nas eqs. (2.15) e (2.31), é sempre possível passar para a decomposição nos vetores de polarização reais com a ajuda de transformações canônicas lineares.

No que se segue, vamos sempre assumir que a soma sobre todos os $\lambda$ 's estará presente e que a transformação canônica linear que conduz à decomposição sobre as polarizações lineares já foi realizada. Verdadeiros $e_{\lambda}$ podem ser escolhidos para serem os mesmos para cada $s$. Em seguida, obtém-se para o vetor $\mathbf{Q}$ em (2.31)

$$
\mathbf{Q}=\sum_{s \in \mathbb{N}, \lambda} \sqrt{\frac{\delta}{\kappa_{s}}}\left(a_{s, \lambda}+a_{s, \lambda}^{+}\right) \mathbf{e}_{\lambda}
$$

onde $\mathbf{e}_{\lambda}=\mathbf{e}_{\lambda}^{*},\left(\mathbf{e}_{\lambda} \mathbf{e}_{\lambda^{\prime}}\right)=\delta_{\lambda, \lambda^{\prime}},\left(\mathbf{n e}_{\lambda}\right)=0$. O Hamiltoniano (2.37), neste caso, é diagonal em relação a $\lambda$ e pode ser escrito como

$$
\begin{aligned}
& \hat{H}=\sum_{k \in \mathbb{N}, s \in \mathbb{N}, \lambda}\left[A_{k s} a_{k, \lambda}^{+} a_{s, \lambda}+\frac{1}{2} B_{k s}\left(a_{k, \lambda} a_{s, \lambda}+a_{s, \lambda}^{+} a_{\zeta, \lambda}^{+}\right)\right] \\
& +\sum_{s \in \mathbb{N}, \lambda} D_{s, \lambda}\left(a_{s, \lambda}+a_{s, \lambda}^{+}\right)+H_{0},
\end{aligned}
$$

com matrizes $A$ e $B$, a coluna $D$, e uma constante real $H_{0}$,

$$
\begin{aligned}
& A_{k s}=\kappa_{k} \delta_{k s}+\frac{\epsilon}{2 \sqrt{\kappa_{k} \kappa_{s}}}, \quad B_{k s}=\frac{\epsilon}{2 \sqrt{\kappa_{k} \kappa_{s}}}, \\
& D_{s, \lambda}=\sqrt{\frac{\epsilon}{2 \Lambda \kappa_{s}}}\left(\mathbf{p e}_{\lambda}\right), \quad H_{0}=\frac{\epsilon}{2} \sum_{s \in \mathbb{N}} \kappa_{s}^{-1}, \quad \epsilon=\frac{\alpha \rho}{\Lambda} .
\end{aligned}
$$


Se a soma sobre $s$ abrange um conjunto infinito de valores, a quantidade $H_{0}$ em (2.44) diverge. Esta é a conhecida divergência logarítmica de $Q E D$. Se os operadores devem ser escritos na forma normal, o termo $H_{0}$ pode ser meramente eliminado da Hamiltoniana (2.43). Se, no entanto, esta exigência não é imposta, a divergência em $H_{0}$, pode ser removida por uma renormalização da massa.

\subsection{Feixes de fótons interagindo com elétrons no campo magnético}

Nesta seção, estudamos as soluções da equação de Dirac com onda plana quantizada (2.14), considerando o potencial envolvido nele, como (2.19), onde $A^{\mu}(x)$ é o operador do potencial de uma onda plana quantizada e $A_{\text {ext }}^{\mu}(x)$ é o potencial do campo magnético externo uniforme e constante na mesma direção de $\mathbf{n}$.

Vamos trabalhar nas variáveis do cone de luz $u^{\mu}$. Se o eixo de $x^{3}$ for escolhido para ser dirigida ao longo do vetor $\mathbf{n}$, então

$$
u^{0}=x^{0}-x^{3}, u^{1}=x^{1}, u^{2}=x^{2}, u^{3}=x^{0}+x^{3} .
$$

Potenciais do campo magnético uniforme constante podem ser escritos nas variáveis do cone de luz como

$$
|e|\left(\mathbf{A}_{\text {ext }}\right)_{\perp}=-|e H| u_{2} \mathbf{e}_{1} .
$$

O seguinte ansatz pode ser utilizado para encontrar soluções da equação de Dirac com onda plana quantizada e o campo magnético externo uniforme

$$
\Psi=U_{1} \mathcal{K}\left\{\sum_{\zeta= \pm 1}[1+\zeta(\boldsymbol{\sigma} \mathbf{n})] \exp \left(i p_{\zeta} u_{0}\right)\right\} \psi v
$$

onde

$$
p_{\zeta}^{\mu}=p^{\mu}+\zeta|e H| n^{\mu}, \quad\left(n p_{\zeta}\right)=(n p)=\Lambda,\left(\mathbf{p}_{\zeta} \mathbf{e}_{1}\right)=\left(\mathbf{p e}_{1}\right), \quad\left(\mathbf{p e}_{2}\right)=0 .
$$

O operador $U_{1}$ é dado como (2.30), ao passo que o operador $\mathcal{K}$ é descrito por

$$
\mathcal{K}=\left(\begin{array}{c}
m+(n p)-\sigma_{3}(\boldsymbol{\sigma} \hat{\mathbf{F}}) \\
(m-(n p)) \sigma_{3}-(\boldsymbol{\sigma} \hat{\mathbf{F}})
\end{array}\right)
$$


na qual se defina

$$
\begin{aligned}
& \boldsymbol{F}=\sum_{\lambda} \mathcal{F}_{\lambda} \mathbf{e}_{\lambda}, \mathcal{F}_{1}=|e H| u_{2}-\left(\mathbf{p e}_{1}\right)-\left(\mathbf{Q e}_{1}\right), \\
& \mathcal{F}_{2}=i \partial_{2}-\left(\mathbf{Q e}_{2}\right), \quad \mathbf{Q}=\sum_{s \in \mathbb{N}} \sum_{\lambda=1,2} \sqrt{\frac{\delta}{\kappa_{s}}}\left(a_{s, \lambda}+a_{s, \lambda}^{+}\right) \mathbf{e}_{\lambda} .
\end{aligned}
$$

Agora, a equação para a função $\psi$ torna-se a equação de Schrödinger estacionária

$$
\begin{aligned}
& p_{0} \psi=\left[\mathcal{H}+\frac{\Lambda}{2}\right] \psi, \\
& \mathcal{H}=\frac{\mathbf{F}^{2}+m^{2}}{2 P}+\sum_{k \in \mathbb{N}} \sum_{\lambda=1,2} \kappa_{k} a_{k, \lambda}^{+} a_{k, \lambda} .
\end{aligned}
$$

Vamos considerar a variável adimensional

$$
\eta=\frac{\sqrt{|e H|} u_{2}-\left(\mathbf{p e}_{1}\right)}{\sqrt{|e H|}},
$$

e os novos operadores de Bose, de criação, $a_{0}^{+}$, e aniquilação, $a_{0}$,

$$
a_{0}^{+}=\frac{1}{\sqrt{2}}\left(\eta-\frac{\partial}{\partial \eta}\right), a_{0}=\frac{1}{\sqrt{2}}\left(\eta+\frac{\partial}{\partial \eta}\right),\left[a_{0} a_{0}^{+}-a_{0}^{+} a_{0}\right]=1 .
$$

Em termos desses operadores, temos

$$
|e H| u_{2}-\left(\mathbf{p e}_{1}\right)=\sqrt{\frac{|e H|}{2}}\left(a_{0}+a_{0}^{+}\right), \partial_{2}=\sqrt{\frac{|e H|}{2}}\left(a_{0}-a_{0}^{+}\right) .
$$

Os operadores $a_{0}$ e $a_{0}^{+}$comutam com cada operador $a_{k, \lambda}$ e $a_{k, \lambda}^{+}$.

Levando em conta as eqs. (2.52), o Hamiltoniano $\mathcal{H}$ em (2.49) representa uma forma quadrática nos operadores de criação $a_{k, \lambda}^{+}$e aniquilação $a_{k, \lambda}$, onde o índice $k$ agora tem os valores $k \in \mathbb{Z}_{+}=0,1,2$, com $a_{0,1}=0$, e $a_{0,2}=a_{0}$ e os números $k \in \mathbb{N}$ novamente indicando os operadores de fótons,

$$
\begin{aligned}
& \mathcal{H}=\sum_{s, k \in \mathbb{Z}_{+} \lambda, \lambda^{\prime}=1,2}\left[A_{s \lambda, k \lambda^{\prime}} a_{s, \lambda}^{+} a_{k, \lambda}+\frac{1}{2}\left(B_{s \lambda, k \lambda^{\prime}}^{*} a_{s, \lambda} a_{k, \lambda^{\prime}}+B_{s \lambda, k \lambda^{\prime}} a_{s, \lambda}^{+} a_{k, \lambda^{\prime}}^{+}\right)\right] \\
& +\frac{m^{2}}{2 \Lambda}-\frac{\omega}{2}+\frac{\epsilon}{2} \sum_{s=1} \kappa_{s}^{-1}, \quad \omega=\frac{|e H|}{\Lambda}, \quad \epsilon=\frac{\alpha \rho}{\Lambda}
\end{aligned}
$$


onde

$$
\begin{aligned}
& A_{s \lambda, k \lambda^{\prime}}=\left[\omega(\lambda-1) \delta_{0, s}+\kappa_{s}\left(1-\delta_{0, s}\right)\right] \delta_{s, k} \delta_{\lambda, \lambda^{\prime}}+B_{s \lambda, k \lambda^{\prime}}, \\
& B_{s \lambda, k \lambda^{\prime}}=\frac{\epsilon\left(1-\delta_{0, s}\right)\left(1-\delta_{0, k}\right) \delta_{\lambda, \lambda^{\prime}}}{2 \sqrt{\kappa_{s} \kappa_{k}}} \\
& +\frac{\sqrt{\alpha \omega}}{2}\left[\frac{(-i)^{\lambda^{\prime}-1}}{\sqrt{\kappa_{k}}}(1-\lambda)\left(1-\delta_{0, k}\right) \delta_{0, s}+\frac{(i)^{\lambda-1}}{\sqrt{\kappa_{s}}}\left(1-\lambda^{\prime}\right)\left(1-\delta_{0, s}\right) \delta_{0, k}\right] .
\end{aligned}
$$

Vamos efetuar a transformação canônica linear

$$
\begin{aligned}
& a=u c-v c^{+}+\gamma, a^{+}=c^{+} u^{+}-c v^{+}+\gamma^{*}, \\
& {\left[c_{k}, c_{s}^{+}\right]_{-}=\delta_{k, s},\left[c_{k}, c_{s}\right]_{-}=\left[c_{k}^{+}, c_{s}^{+}\right]_{-}=0 .}
\end{aligned}
$$

O fato de que a transformação é canônica e, em particular, a exigência de que os novos operadores $c_{k}^{+}$e $c_{k}$ sejam de novo operadores de criação e aniquilação sujeitos às relações de comutação de Bose impõem as seguintes condições nas matrizes $u$ e $v$, e a coluna $\gamma$ :

$$
\begin{aligned}
& u u^{+}-v v^{+}=1, v u^{T}-u v^{T}=0, \\
& u^{+} u-v^{T} v^{*}=1, v^{T} u^{*}-u^{+} v=0,
\end{aligned}
$$

veja [42]. As relações (2.55) podem ser resolvidas pelos operadores $c_{k}^{+}$e $c_{k}$, da seguinte forma

$$
\begin{aligned}
& c=u^{+} a+v^{T} a^{+}-u^{+} \gamma-v^{T} \gamma^{*}, \\
& c^{+}=a^{+} u+a v^{*}-\gamma^{*} u-\gamma v^{*} .
\end{aligned}
$$

Para diagonalizar o Hamiltoniano $\mathcal{H}$, os parâmetros da transformação têm 
que ser tomados como

$$
\begin{aligned}
& u_{s \lambda, k \lambda^{\prime}}=\left[\left(\sqrt{\frac{r_{k \lambda^{\prime}}}{\kappa_{s}}}+\sqrt{\frac{\kappa_{s}}{r_{k \lambda^{\prime}}}}\right) \frac{\left(3-2 \lambda^{\prime}\right)(2-\lambda)-i(\lambda-1)}{2\left(r_{k \lambda^{\prime}}^{2}-\kappa_{s}^{2}\right)}\left(1-\delta_{0, s}\right)\right. \\
& \left.+\delta_{0, s}(\lambda-1)\left(\lambda^{\prime}-1\right) \sqrt{\frac{\omega}{\alpha r_{k \lambda^{\prime}}^{3}}}\right]\left[1+\left(\lambda^{\prime}-2\right) \delta_{0, k}\right] q_{k \lambda^{\prime}}, \\
& v_{s \lambda, k \lambda^{\prime}}=\left[\left(\sqrt{\frac{r_{k \lambda^{\prime}}}{\kappa_{s}}}-\sqrt{\frac{\kappa_{s}}{r_{k \lambda^{\prime}}}}\right) \frac{\left(3-2 \lambda^{\prime}\right)(2-\lambda)-i(\lambda-1)}{2\left(r_{k \lambda^{\prime}}^{2}-\kappa_{s}^{2}\right)}\left(1-\delta_{0, s}\right)\right. \\
& \left.+\delta_{0, s}(1-\lambda)\left(2-\lambda^{\prime}\right) \sqrt{\frac{\omega}{\alpha r_{k \lambda^{\prime}}^{3}}}\right]\left[1+\left(\lambda^{\prime}-2\right) \delta_{0, k}\right] q_{k \lambda^{\prime}}, \\
& q_{k \lambda}=\left[\frac{(-1)^{\lambda} \omega}{r_{k \lambda}^{3} \epsilon}+2 \sum_{s \in \mathbb{N}}\left(r_{k \lambda}^{2}-\kappa_{s}^{2}\right)^{-2}\right]^{-1 / 2}, \gamma_{k \lambda}=0 .
\end{aligned}
$$

As equações (2.59) são válidas para todas as $s, \lambda, k, \lambda^{\prime}$, menos $s, \lambda, 0,1$; se $k, \lambda^{\prime}=0,1$, então, pela definição, estabelecemos

$$
u_{s, \lambda, 0,1}=v_{s, \lambda, 0,1}=q_{01}=0 .
$$

As quantidades $r_{k \lambda}$ são raízes positivas da equação

$$
\sum_{s} \frac{\epsilon}{r_{k \lambda}^{2}-\kappa_{s}^{2}}=1+\frac{(3-2 \lambda) \omega}{r_{k \lambda}}, r_{01}=0 ; \epsilon=\frac{\alpha \rho}{\Lambda} .
$$

O Hamiltoniano transformado é

$$
\begin{aligned}
& \hat{H}=\tilde{H}_{e}+\tilde{H}_{\gamma}, \quad \tilde{H}_{e}=r_{02} c_{0}^{+} c_{0}+\frac{m^{2}}{2 \Lambda}-\frac{\omega}{2}, \\
& \tilde{H}_{\gamma}=\sum_{s \in \mathbb{N} ; \lambda=1,2} r_{s \lambda} c_{s, \lambda}^{+} c_{s, \lambda}+\tilde{H}_{\gamma 0}, \\
& \tilde{H}_{\gamma 0}=-\sum_{s, k \in \mathbb{N} ; \lambda, \lambda^{\prime}} r_{k \lambda^{\prime}}\left|v_{s \lambda, k \lambda^{\prime}}\right|^{2}+\frac{\epsilon}{2} \sum_{s \in \mathbb{N}} \kappa_{s}^{-1},
\end{aligned}
$$

onde $p_{0}$ é uma energia do elétron e $p_{z}$ é a projeção em $z$ do momento do elétron, de tal forma que para os estados do elétron $\Lambda>0$.

Os estados estacionários do sistema são:

$$
\Psi=\Psi_{\gamma} \otimes \Psi_{e},
$$


onde $\Psi_{\gamma}$ é o vetor de estado dos quase-fótons,

$$
\Psi_{\gamma}=\prod_{s \in \mathbb{N}, \lambda_{1}=1,2} \frac{\left(c_{s, \lambda_{1}}^{+}\right)^{N_{s, \lambda_{1}}}}{\sqrt{N_{s, \lambda_{1}} !}}|0\rangle_{c} .
$$

Aqui, $c_{s \lambda}|0\rangle_{c}=0, s \in \mathbb{N}, \forall \lambda$ e $\Psi_{e}$ é o vetor de estado do quase-elétron, a forma explícita que não é importante para os nossos propósitos.

Fisicamente, o estado (2.62) representa um sistema de um elétron colocado no nível de Landau com energia cíclotron ligeiramente modificada $r_{02}$ e um sistema independente de quase-fótons com frequências $r_{k \lambda}$. Nessa equação, $N_{k, \lambda}, \lambda=1,2$ são números de ocupação de tais fótons. As frequências diferem das frequências dos fótons livres. As diferenças dependem do campo magnético $H$, da projeção do momento dos elétrons $\Lambda$, da densidade de elétrons $\rho=1 / V$ e da constante de estrutura fina, $\alpha$.

Para os nossos propósitos, é necessário ter, nesse caso, explicitamente, apenas as matrizes $u_{s \lambda, k \lambda^{\prime}}$ que correspondem à transformação dos operadores dos fótons, i.e., as matrizes com os índices $s, k \in \mathbb{N}$, e $\lambda=1$, 2. Estas matrizes têm a forma

$$
\begin{aligned}
& u_{s \lambda, k \lambda^{\prime}}=\left[\left(\sqrt{\frac{r_{k \lambda^{\prime}}}{\kappa_{s}}}+\sqrt{\frac{\kappa_{s}}{r_{k \lambda^{\prime}}}}\right) \frac{(-1)^{\lambda^{\prime}-1} \delta_{\lambda, 1}-i \delta_{\lambda, 2}}{2\left(r_{k \lambda^{\prime}}^{2}-\kappa_{s}^{2}\right)}\right] q_{k \lambda^{\prime}} \\
& =\frac{\left[(-1)^{\lambda^{\prime}-1} \delta_{\lambda, 1}-i \delta_{\lambda, 2}\right]}{2\left(r_{k \lambda^{\prime}}-\kappa_{s}\right) \sqrt{r_{k \lambda^{\prime}} \kappa_{s}}} q_{k \lambda^{\prime}}, \\
& q_{k \lambda}=\left[\frac{(-1)^{\lambda} \omega}{r_{k \lambda}^{3} \epsilon}+2 \sum_{s \in \mathbb{N}}\left(r_{k \lambda}^{2}-\kappa_{s}^{2}\right)^{-2}\right]^{-1 / 2} .
\end{aligned}
$$

No que se segue, vamos considerar situações em que o parâmetro $\epsilon$ é pequeno. É por isso que vamos manter apenas os termos com a menor potência de $\epsilon$. Nesse caso,

$$
\begin{aligned}
& r_{k \lambda} \approx \kappa_{k}+\frac{\epsilon}{2\left[\kappa_{k}+(3-2 \lambda) \omega\right]}, \quad \kappa_{s}=\kappa_{0} s, \kappa_{0}=2 \pi \rho^{1 / 3}, \\
& u_{s \lambda, k \lambda^{\prime}}=\frac{\left[(-1)^{\lambda^{\prime}-1} \delta_{\lambda, 1}-i \delta_{\lambda, 2}\right]}{2\left(\kappa_{k}-\kappa_{s}\right) \sqrt{\kappa_{k} \kappa_{s}}} q_{k \lambda^{\prime}}, \quad s \neq k, \\
& u_{k \lambda, k \lambda^{\prime}}=\epsilon^{-1}\left[1+(3-2 \lambda) \frac{\omega}{\kappa_{k}}\right]\left[(-1)^{\lambda^{\prime}-1} \delta_{\lambda, 1}-i \delta_{\lambda, 2}\right] q_{k \lambda^{\prime}}, s=k .
\end{aligned}
$$




\section{Capítulo 3}

\section{Emaranhamento em sistemas quânticos compostos}

O emaranhamento quântico é um fenômeno da mecânica quântica em que os estados quânticos de dois ou mais objetos têm de ser descritos com referência um ao outro, mesmo que os objetos individuais possam ser espacialmente separados. Isso leva à correlação entre as propriedades físicas observáveis dos sistemas. Por exemplo, é possível preparar duas partículas num estado quântico de tal modo que quando uma é observada para ser virada, a outra irá sempre ser observada para ser rodada para baixo e vice-versa.

Isso, apesar do fato de que é impossível de prever, de acordo com a mecânica quântica, que conjunto de medidas será observado. Essa interdependência é mantida, mesmo que essas instalações sejam separadas no espaço para além de quaisquer interações conhecidas, é uma contradição lógica com o princípio da localidade. Por exemplo, você pode obter um par de fótons em um estado emaranhado, e, em seguida, se a medição da helicidade da primeira partícula for positiva, a helicidade da segunda partícula vai ser sempre negativa, e vice-versa. Como resultado, as medições executadas em um sistema parecem influenciar instantaneamente outros sistemas complicados como ele. Mas emaranhamento quântico não permite a transmissão de informação clássica mais rápida que a velocidade da luz.

O emaranhamento quântico tem aplicações nas tecnologias emergentes de computação quântica e criptografia quântica, e tem sido utilizado para realizar o teletransporte quântico experimentalmente. Há diferentes pontos de vista sobre o processo de emaranhamento, relacionados às diferentes interpretações da mecânica quântica, que não serão tratados neste trabalho. 


\subsection{Descrição dos estados quânticos dos sis- temas físicos}

Sabemos que um estado quântico corresponde a um conjunto de cópias idênticas do mesmo sistema físico [9, 10, 11, 12, 13, 14, 15, 16, 31]. Se as condições externas definem os estados das cópias de forma maximal, o estado quântico é dito estado puro e é dado por um vetor $|\Psi\rangle$ do estado de Hilbert $\mathcal{H}$, $(|\Psi\rangle \in \mathcal{H})$. No caso geral, as condições externas não definem os estados das cópias de forma maximalmente completa. Nesse caso, o estado quântico é misto e é dado por um operador estatístico $\hat{\rho}$. O operador estatístico que descreve um estado puro $|\Psi\rangle$ é o operador de projeção, $\hat{\rho}=\hat{P}_{\Psi}=|\Psi\rangle\langle\Psi|$. Para sistematizar o formalismo do operador estatístico, é útil fazer a seguinte consideração:

O estado quântico $\rho$ define os valores médios de todas as grandezas físicas medidas em conjunto. Como sabemos, uma grandeza física $A$ é dada na teoria quântica por um operador autoadjunto $\hat{A}$. Então, deve existir uma correspondência ${ }^{1}$

$$
\hat{A} \stackrel{\rho}{\rightarrow}\langle A\rangle=\Phi_{\rho}[\hat{A}]=r \in \mathbb{R},
$$

onde $\Phi_{\rho}(\hat{A})$ é um funcional definido no conjunto dos operadores e cuja imagem está contida nos números reais. O funcional $\Phi_{\rho}(\hat{A})$ deve ser linear porque $\langle A+B\rangle=\langle A\rangle+\langle B\rangle$. O análogo do teorema Riesz para funcionais definidos no espaço de operadores afirma que qualquer funcional linear desse tipo pode ser representado como o traço na forma

$$
\Phi_{\rho}[\hat{A}]=\operatorname{tr} \hat{A} \hat{\rho}
$$

onde $\hat{\rho}$ é um operador que define o funcional $\Phi[\hat{A}]$. Para providenciar valores médios reais, o operador $\hat{\rho}$ deve ser autoadjunto, $\hat{\rho}^{+}=\hat{\rho}$. Justificando Eq. (3.2), suponhamos que $\left|\varphi_{n}\right\rangle=|n\rangle$ seja um sistema completo e ortonormal,

\footnotetext{
${ }^{1}$ Para simplicidade, consideramos o caso unidimensional.
} 
$\langle n \mid m\rangle=\delta_{n m}$. Introduzimos o "projetor com rotação" $\hat{P}_{m n}=|m\rangle\langle n|$. Temos

$$
\left.\begin{array}{l}
\hat{A}=\sum_{n, m}|m\rangle\langle m|\hat{A}| n\rangle\langle n|=\sum_{n, m}\langle m|\hat{A}| n\rangle \hat{P}_{m n} \\
\operatorname{tr} \hat{A} \hat{P}_{m n}=\sum_{k}\left\langle k\left|\hat{A} \hat{P}_{m n}\right| k\right\rangle=\sum_{k}\langle k|\hat{A}| m\rangle\langle n|| k\rangle=\langle n|\hat{A}| m\rangle
\end{array}\right\}
$$

Usando a linearidade de $\Phi[\hat{A}]$, obtemos

$$
\begin{aligned}
& \Phi[\hat{A}]=\sum_{n, m} \Phi\left[\hat{P}_{m n}\right] \operatorname{tr} \hat{A} \hat{P}_{n m}=\operatorname{tr} \hat{A} \hat{\rho} \\
& \hat{\rho}=\sum_{n, m} \Phi\left[\hat{P}_{m n}\right] \hat{P}_{n m}=\sum_{n, m}\langle m|\hat{\rho}| n\rangle \hat{P}_{m n} .
\end{aligned}
$$

A equação

$$
\Phi\left[\hat{P}_{n m}\right]=\langle n|\hat{\rho}| m\rangle
$$

estabelece uma relação unívoca entre $\Phi[\hat{A}]$ e o operador $\hat{\rho}$ (através dos seus elementos de matriz). É possível provar que $\hat{\rho}$ não depende da escolha do conjunto $|n\rangle$.

O operador $\hat{\rho}$ se chama operador estatístico. No caso geral, o estado de um conjunto é definido por um operador estatístico.

Suponhamos que o operador $\hat{\rho}$ tem espectro discreto $\hat{\rho}\left|\lambda_{\alpha}\right\rangle=\lambda_{\alpha}\left|\lambda_{\alpha}\right\rangle$. Neste caso, temos a representação espectral

$$
\hat{\rho}=\sum_{n} \lambda_{\alpha} \hat{P}_{\lambda_{\alpha}}, \quad \hat{P}_{\lambda_{\alpha}}=\left|\lambda_{\alpha}\right\rangle\left\langle\lambda_{\alpha}\right|
$$

e o valor médio da grandeza física $A$ é

$$
\langle A\rangle=\Phi_{\rho}[\hat{A}]=\operatorname{tr} \hat{A} \hat{\rho}=\sum_{n} \lambda_{n}\left\langle\lambda_{n}|\hat{A}| \lambda_{n}\right\rangle .
$$

Os estados puros $|\lambda\rangle$ são descritos por operadores estatísticos que são operadores de projeção.

$$
\hat{P}_{\lambda}=|\lambda\rangle\langle\lambda| .
$$

Se o operador estatístico não é um operador de projeção, o estado quântico é misto. 
Os elementos de matriz do operador estatístico $\rho_{n m}=\langle n|\hat{\rho}| m\rangle$ chamamse matriz de densidade. Os valores médios podem ser expressos através da matriz de densidade,

$$
\langle A\rangle=\operatorname{tr} \hat{A} \hat{\rho}=\sum_{n, m}\langle n|\hat{A}| m\rangle\langle m|\hat{\rho}| n\rangle=\sum_{n, m}\langle n|\hat{A}| m\rangle \rho_{m n} .
$$

Na representação de coordenadas, a matriz de densidade tem a forma $\langle x|\hat{\rho}| y\rangle=$ $\rho(x, y)$. Neste caso

$$
\begin{aligned}
& \langle A\rangle=\operatorname{tr} \hat{A} \hat{\rho}=\int\langle y|\hat{A} \hat{\rho}| y\rangle d y \\
& =\int\langle y|\hat{A}| x\rangle\langle x|\hat{\rho}| y\rangle d y d x=\int\langle y|\hat{A}| x\rangle \rho(x, y) d y d x
\end{aligned}
$$

É possível provar as seguintes propriedades do operador de densidade, veja $[15]$ :

I. O operador estatístico é autoadjunto, $\hat{\rho}^{+}=\hat{\rho}$.

II. O operador estatístico é positivo, $\hat{\rho}>0$, o que significa

$$
\langle\varphi|\hat{\rho}| \varphi\rangle \geq 0 \forall|\varphi\rangle \text {. }
$$

III. O operador estatístico é normalizado tr $\hat{\rho}=1$.

IV. Para o operador $\hat{\rho}^{2}$, temos $\operatorname{tr} \hat{\rho}^{2} \leq 1$.

V. Temos o critério

$$
\begin{aligned}
& \operatorname{tr} \hat{\rho}^{2}=1 \quad \text { o estado é puro, } \\
& \operatorname{tr} \hat{\rho}^{2}<1 \quad \text { o estado é misto. }
\end{aligned}
$$

A evolução temporal dos estados mistos é dada pela equação de Liouvillevon Neumann

$$
i \hbar \frac{\partial \hat{\rho}(t)}{\partial t}=\hat{H} \hat{\rho}(t)-\hat{\rho}(t) \hat{H}=[\hat{H}, \hat{\rho}(t)]
$$

Considere um operador $\hat{A}$ com espectro discreto $\hat{A}\left|a_{i}\right\rangle=a_{i}\left|a_{i}\right\rangle$. Se um estado $\hat{\rho}$ é puro, $\hat{\rho}=\hat{P}_{\psi}=|\psi\rangle\langle\psi|$, a probabilidade de achar o valor $a_{i}$ na medida da grandeza $A$ é

$$
\mathcal{P}_{i}=\left|\left\langle a_{i} \mid \psi\right\rangle\right|^{2}=\left\langle a_{i}|\hat{\rho}| a_{i}\right\rangle
$$


No caso dos estados mistos, $\hat{\rho}=\sum_{n} \lambda_{n} \hat{P}_{\lambda_{n}}$, a probabilidade de achar o valor $a_{i}$ na medida da grandeza $A$ é

$$
\mathcal{P}_{i}=\sum_{n} \lambda_{n}\left|\left\langle a_{i} \mid \lambda_{n}\right\rangle\right|^{2}=\left\langle a_{i}|\hat{\rho}| a_{i}\right\rangle .
$$

Se o espectro é contínuo, no caso dos estados puros $\hat{\rho}=\hat{P}_{\psi}$, a probabilidade de achar o valor entre $x$ e $x+d x$ é

$$
d \mathcal{P}(x)=|\langle x \mid \psi\rangle|^{2} d x=\langle x|\hat{\rho}| x\rangle d x .
$$

No caso dos estados mistos, $\hat{\rho}=\sum_{n} \lambda_{n} \hat{P}_{\lambda_{n}}$, a probabilidade de achar o valor entre $x$ e $x+d x$ é

$$
d \mathcal{P}(x)=\sum_{n} \lambda_{n}\left(\left|\left\langle x \mid \lambda_{n}\right\rangle\right|^{2} d x\right)=\langle x|\hat{\rho}| x\rangle d x .
$$

Como um exemplo importante, consideramos um conjunto de sistemas idênticos em equilíbrio térmico, sobre condições externas de um reservatório com a temperatura absoluta $T$. É possível mostrar que o operador estatístico neste caso é

$$
\hat{\rho}=Z^{-1} e^{-\beta \hat{H}}, \beta^{-1}=k T,
$$

onde $k$ é a constante de Boltsmann e $\hat{H}$, o Hamiltoniano do sistema, $Z$ é uma constante de normalização,

$$
\operatorname{tr} \rho=1 \Longrightarrow Z=\operatorname{tr} e^{-\beta \hat{H}}
$$

Às vezes, $Z$ se chama função de partição do sistema em equilíbrio térmico.

Vamos introduzir o operador de entropia usando a definição de von Neumann $^{2}$,

$$
\hat{S}=-\log \hat{\rho}=-\log \sum_{n} \lambda_{n} P_{\lambda_{n}} .
$$

Em decorrência das propriedades do operador densidade, anteriormente estudadas, podemos também afirmar que o operador entropia é auto-adjunto e não-negativo. Em outras palavras, os autovalores do operador entropia são reais e não-negativos. Mas, ainda da mesma forma que o operador densidade, o operador entropia satisfaz a equação de Von Neumann, ou seja:

$$
i \hbar \frac{d \hat{S}}{d t}=[\hat{H}, \hat{S}] .
$$

\footnotetext{
${ }^{2}$ Note que usamos o símbolo log para indicar $\log _{2}$ exceto se for indicado outro.
} 
O operador de entropia satisfaz a aditividade, pois se $\hat{\rho}$ descreve conjuntos independentes, ele é produto direto dos operadores $\hat{\rho}_{1}$ e $\hat{\rho}_{2}$ nos espaços vetoriais correspondentes e, portanto, pode-se demonstrar neste caso que:

$$
\hat{S}\left(\hat{\rho}_{1} \otimes \hat{\rho}_{2}\right)=\hat{S}\left(\hat{\rho}_{1}\right)+\hat{S}\left(\hat{\rho}_{2}\right),
$$

que representa a propriedade de aditividade.

O valor médio do operador entropia é dado num conjunto caracterizado pelo operador densidade $\hat{\rho}$ por:

$$
S=\langle\hat{S}\rangle=-\langle\log \hat{\rho}\rangle=-\operatorname{tr}(\hat{\rho} \log \hat{\rho})
$$

e é chamado de Entropia de Informação, ou Entropia de Von Neumann.

Esta formula pode ser modificada assim:

$$
\begin{aligned}
\hat{\rho} & =\sum_{n} \lambda_{n} \hat{P}_{\lambda_{n}}, \hat{\rho} \log \hat{\rho}=\sum_{n} \lambda_{n}\left(\log \lambda_{n}\right) \hat{P}_{\lambda_{n}}, \operatorname{tr} \hat{P}_{\lambda_{n}}=1 \\
\Longrightarrow & S=-\operatorname{tr}(\hat{\rho} \log \hat{\rho})=-\sum_{n} \lambda_{n}\left(\log \lambda_{n}\right) .
\end{aligned}
$$

No caso de um conjunto puro, $\lambda_{n}=1, \lambda_{m}=0 \forall m \neq n$, temos $S=0$.

Vamos chamar estado completamente misto se $\lambda_{n}=1 / d, \forall n$, onde $d$ é a dimensão do espaço de Hilbert. Em seguida

$$
S=\log d
$$

\subsection{Sistemas quânticos compostos do numero finito de qubits}

Um sistema quântico se chama composta se ele consiste de $N<\infty$ subsistemas $A_{1}, A_{2}, \ldots A_{N}$ e seu espaço de Hilbert $\mathcal{H}$ é o produto tensorial de espaços $\mathcal{H}_{A_{i}}$, do todos os subsistemas, i.e.,

$$
\mathcal{H}=\bigotimes_{i=1}^{N} \mathcal{H}_{A_{i}}=\mathcal{H}_{A_{1}} \otimes \mathcal{H}_{A_{2}} \otimes \cdots \otimes \mathcal{H}_{A_{N}}
$$

No nosso estudo, consideramos somente sistemas $A_{i}$ com espaços de Hilbert de dimensão finita, i.e., $\operatorname{dim} \mathcal{H}_{A_{i}}<\infty$ tal que $\operatorname{dim} \mathcal{H}<\infty$. Em particular, 
somente sistemas $A_{i}$ com espaços de Hilbert de dimensão dois. Uma partícula de spin $1 / 2$ representa o exemplo mais simples de um sistema quântico com um espaço de Hilbert de dimensão dois complexo $\mathcal{H}=\mathbb{C}^{2}$. Ao mesmo tempo, o sistema é chamado de qubit. Outro exemplo de um qubit é um fóton com frequência fixa com duas polarizações diferentes.

Vejamos alguns vetores base e sistemas ortogonais completos nos espaços considerados.

Os kets $|0\rangle=|\uparrow\rangle$ e $|1\rangle=|\downarrow\rangle$ representam estados de spin para cima e para baixo, respectivamente, ao longo da direção $z$,

$$
\begin{aligned}
& |0\rangle=|\uparrow\rangle=\left(\begin{array}{l}
1 \\
0
\end{array}\right),|1\rangle=|\downarrow\rangle=\left(\begin{array}{c}
0 \\
1
\end{array}\right), \\
& \sigma_{z}|\uparrow\rangle=|\uparrow\rangle, \sigma_{z}|\downarrow\rangle=-|\downarrow\rangle, \sigma_{z}=\left(\begin{array}{cc}
1 & 0 \\
0 & -1
\end{array}\right) .
\end{aligned}
$$

Temos as relações de ortonormalização

$$
\left\langle\alpha \mid \alpha^{\prime}\right\rangle=\delta_{\alpha \alpha^{\prime}}, \quad \alpha, \alpha^{\prime}=0,1
$$

Dessa forma, Eqs. (3.12) e (3.13) descrevem a base ortonormal para um qubit.

No que se segue, restringimos nossa consideração a sistemas compostos de muitos (N) qubits.

Sejam $\left\{|\alpha\rangle_{A_{i}}, \alpha=0,1\right\}$ bases ortonormais em espaços $\mathcal{H}_{A_{i}}, i=1, \ldots, N$, de cada qubit,

$$
{ }_{A_{i}}\left\langle\alpha \mid \alpha^{\prime}\right\rangle_{A_{i}}=\delta_{\alpha \alpha^{\prime}}, \quad i=1, \ldots, N
$$

Nesse caso,

$$
\left|\alpha_{1}, \alpha_{2}, \ldots, \alpha_{N}\right\rangle=\bigotimes_{i=1}^{N}|\alpha\rangle_{A_{i}}
$$

é uma base no espaço total $\mathcal{H}$,

$$
\left\langle\alpha_{1}, \alpha_{2}, \ldots, \alpha_{N} \mid \alpha_{1}^{\prime}, \alpha_{2}^{\prime}, \ldots, \alpha_{N}^{\prime}\right\rangle=\prod_{i=1}^{N} \delta_{\alpha_{i} \alpha_{i}^{\prime}} .
$$

Qualquer estado puro $|\Psi\rangle \in \mathcal{H}$ pode ser decomposto nesta base como

$$
|\Psi\rangle=\sum_{\alpha_{1}=0,1} \cdots \sum_{\alpha_{N}=0,1} \Psi_{\alpha_{1}, \alpha_{2}, \ldots, \alpha_{N}}\left|\alpha_{1}, \alpha_{2}, \ldots, \alpha_{N}\right\rangle
$$


onde $\Psi_{\alpha_{1}, \alpha_{2}, \ldots, \alpha_{N}}$ são certos coeficientes.

O produto escalar de dois vetores $|\Psi\rangle \in \mathcal{H}$ e $\left|\Psi^{\prime}\right\rangle \in \mathcal{H}$, onde

$$
\left|\Psi^{\prime}\right\rangle=\sum_{\alpha_{1}=0,1} \cdots \sum_{\alpha_{N}=0,1} \Psi_{\alpha_{1}, \alpha_{2}, \ldots, \alpha_{N}}^{\prime}\left|\alpha_{1}, \alpha_{2}, \ldots, \alpha_{N}\right\rangle
$$

tem a seguinte forma

$$
\left\langle\Psi \mid \Psi^{\prime}\right\rangle=\sum_{\alpha_{1}=0,1} \cdots \sum_{\alpha_{N}=0,1} \Psi_{\alpha_{1}, \alpha_{2}, \ldots, \alpha_{N}}^{*} \Psi_{\alpha_{1}, \alpha_{2}, \ldots, \alpha_{N}}^{\prime}
$$

tal que a condição de normalização é

$$
\langle\Psi \mid \Psi\rangle=1 \Longrightarrow \sum_{\alpha_{1}=0,1} \cdots \sum_{\alpha_{N}=0,1}\left|\Psi_{\alpha_{1}, \alpha_{2}, \ldots, \alpha_{N}}\right|^{2}=1 .
$$

Suponha que temos um estado geral $\hat{\rho}$ de um sistema bipartido e estamos interessados apenas nos estados dos subsistemas $A$ ou $B$. Temos que calcular os operadores de densidade reduzida $\hat{\rho}_{A}$ e $\hat{\rho}_{B}$, realizando o traço sobre os subsistemas $B$ ou $A$.

$$
\hat{\rho}_{A}=\operatorname{tr}_{B} \hat{\rho}, \quad \hat{\rho}_{B}=\operatorname{tr}_{A} \hat{\rho} .
$$

Usando as bases $|\alpha\rangle_{A}$ e $\left.|\beta\rangle_{B}\right\}$ (veja (3.14) e (3.15)), podemos escrever

$$
\hat{\rho}_{A}=\operatorname{tr}_{B} \hat{\rho}=\sum_{\beta}\langle\beta|\hat{\rho}| \beta\rangle, \quad \hat{\rho}_{B}=\operatorname{tr}_{A} \hat{\rho}=\sum_{\alpha}\langle\alpha|\hat{\rho}| \alpha\rangle .
$$

Os traços parciais são ainda operadores.

Em termos de matriz de densidade do sistema total,

$$
\hat{\rho}\left(\alpha, \beta \mid \alpha^{\prime}, \beta^{\prime}\right)=\left\langle\alpha, \beta|\hat{\rho}| \alpha^{\prime}, \beta^{\prime}\right\rangle,
$$

o procedimento da redução produz as matrizes de densidade $\hat{\rho}_{A}\left(\alpha \mid \alpha^{\prime}\right)$ e $\hat{\rho}_{B}\left(\beta \mid \beta^{\prime}\right)$ reduzidas,

$$
\hat{\rho}_{A}\left(\alpha \mid \alpha^{\prime}\right)=\sum_{\beta}\left\langle\alpha, \beta|\hat{\rho}| \alpha^{\prime}, \beta\right\rangle, \quad \hat{\rho}_{B}\left(\beta \mid \beta^{\prime}\right)=\sum_{\alpha}\left\langle\alpha, \beta|\hat{\rho}| \alpha, \beta^{\prime}\right\rangle .
$$




\subsection{Estados separáveis e emaranhados}

O estado puro $|\Psi\rangle$ de $N$ subsistemas $A_{1}, A_{2}, \ldots A_{N}$ é completamente separável se, e somente se, ele pode ser representado como:

$$
|\Psi\rangle=\left|\Psi_{A_{1}}\right\rangle \otimes\left|\Psi_{A_{2}}\right\rangle \otimes \cdots \otimes\left|\Psi_{A_{N}}\right\rangle .
$$

De forma correspondente, o estado $|\Psi\rangle$ é emaranhado se ele não é separável.

O estado misto $\hat{\rho}$ de $N$ subsistemas $A_{1}, A_{2}, \ldots A_{N}$ é completamente separável se, e somente se, ele pode ser representado como:

$$
\hat{\rho}=\sum_{j=0}^{m} p_{j}\left(\hat{\rho}_{A_{1}}^{j} \otimes \hat{\rho}_{A_{2}}^{j} \cdots \otimes \hat{\rho}_{A_{N}}^{j}\right),
$$

onde

$$
p_{j}>0, \sum_{j=0}^{m} p_{j}=1,
$$

e $\hat{\rho}_{A_{k}}^{j}$ são operadores estatísticos de subsistemas $A_{k}, k=1, \ldots, N$. De forma correspondente, o estado $\hat{\rho}$ é emaranhado se ele não é separável. Um exemplo de estado misto não emaranhado é

$$
\hat{\rho}=\frac{1}{2}[|00\rangle\langle 00|+| 11\rangle\langle 11|]
$$

\subsection{Sistemas de dois qubits}

Consideremos o sistema de dois qubits $A$ e $B$, por exemplo, duas partículas de spin $1 / 2$, para as quais o espaço de Hilbert é dado por $\mathcal{H}=\mathcal{H}_{A} \otimes \mathcal{H}_{B}=$ $\mathbb{C}^{2} \otimes \mathbb{C}^{2}$. Como já foi mencionado, o sistema de dois fótons com frequências diferentes e com dois estados de polarização em cada fóton é matematicamente equivalente ao sistema de dois spins. Nesse caso, consideramos diferentes bases para o sistema de dois qubits e exemplos de manipulação de estados desse sistema.

Se o estado de um sistema composto (bipartido) é puro, pode ser representado por meio de uma base $|a, b\rangle=|a\rangle_{A} \otimes|b\rangle_{B}$ da seguinte forma:

$$
|\Psi\rangle=\sum_{\alpha, b=0,1} \Psi_{a b}|a\rangle \otimes|b\rangle
$$


onde os sub-índices $A$ e $B$ foram omitidos para simplificar.

Em primeiro lugar, lembremos algumas noções do produto tensorial de espaços vetoriais tendo em mente nossos dois espaços 2-dimensionais $\mathbb{C}^{2}$ com produto tensorial 4-dimensional $\mathbb{C}^{2} \otimes \mathbb{C}^{2}$. Considere

$$
|a\rangle=\left(\begin{array}{c}
a_{1} \\
a_{2}
\end{array}\right) \in \mathcal{H}_{A} \text { e }|b\rangle=\left(\begin{array}{c}
b_{1} \\
b_{2}
\end{array}\right) \in \mathcal{H}_{B}
$$

Logo,

$$
\begin{aligned}
& |a\rangle \otimes|b\rangle=\left(\begin{array}{l}
a_{1} \\
a_{2}
\end{array}\right) \otimes\left(\begin{array}{l}
b_{1} \\
b_{2}
\end{array}\right) \\
& =\left(\begin{array}{ll}
a_{1} & \left(\begin{array}{l}
b_{1} \\
b_{2} \\
b_{1} \\
b_{2}
\end{array}\right) \\
a_{2} &
\end{array}\right)=\left(\begin{array}{l}
a_{1} b_{1} \\
a_{1} b_{2} \\
a_{2} b_{1} \\
a_{2} b_{2}
\end{array}\right) .
\end{aligned}
$$

Primeiro, escrevemos abaixo a chamada base computacional $\{|00\rangle,|01\rangle,|10\rangle,|11\rangle\}$, em $\mathcal{H}$,

$$
\begin{aligned}
& \left|\Theta_{1}\right\rangle=|00\rangle=|\uparrow\rangle \otimes|\uparrow\rangle=\left(\begin{array}{l}
1 \\
0
\end{array}\right) \otimes\left(\begin{array}{l}
1 \\
0
\end{array}\right)=\left(\begin{array}{l}
1 \\
0 \\
0 \\
0
\end{array}\right), \\
& \left|\Theta_{2}\right\rangle=|01\rangle=|\uparrow\rangle \otimes|\downarrow\rangle=\left(\begin{array}{l}
1 \\
0
\end{array}\right) \otimes\left(\begin{array}{l}
0 \\
1
\end{array}\right)=\left(\begin{array}{l}
0 \\
1 \\
0 \\
0
\end{array}\right), \\
& \left|\Theta_{3}\right\rangle=|10\rangle=|\downarrow\rangle \otimes|\uparrow\rangle=\left(\begin{array}{l}
0 \\
1
\end{array}\right) \otimes\left(\begin{array}{l}
1 \\
0
\end{array}\right)=\left(\begin{array}{l}
0 \\
0 \\
1 \\
0
\end{array}\right), \\
& \left|\Theta_{4}\right\rangle=|11\rangle=|\downarrow\rangle \otimes|\downarrow\rangle=\left(\begin{array}{l}
0 \\
1
\end{array}\right) \otimes\left(\begin{array}{l}
0 \\
1
\end{array}\right)=\left(\begin{array}{l}
0 \\
0 \\
1
\end{array}\right) .
\end{aligned}
$$


O produto tensorial $|a\rangle \otimes\langle b|$ é uma matriz $R=\left(R_{i k}\right)$,

$$
\begin{aligned}
& R_{i k}=a_{i} b_{k}^{*}=\left(\begin{array}{l}
a_{1} \\
a_{2}
\end{array}\right) \otimes\left(\begin{array}{ll}
b_{1}^{*} & b_{2}^{*}
\end{array}\right)= \\
& =\left(\begin{array}{ll}
a_{1} b_{1}^{*} & a_{1} b_{2}^{*} \\
a_{2} b_{1}^{*} & a_{2} b_{2}^{*}
\end{array}\right) .
\end{aligned}
$$

Pode-se ver facilmente que

$$
\begin{aligned}
& |0\rangle\left\langle 0\left|=\left(\begin{array}{ll}
1 & 0 \\
0 & 0
\end{array}\right), \quad\right| 1\right\rangle\langle 1|=\left(\begin{array}{ll}
0 & 0 \\
0 & 1
\end{array}\right), \\
& |0\rangle\left\langle 1\left|=\left(\begin{array}{ll}
0 & 1 \\
0 & 0
\end{array}\right), \quad\right| 1\right\rangle\langle 0|=\left(\begin{array}{ll}
0 & 0 \\
1 & 0
\end{array}\right) .
\end{aligned}
$$

O produto tensorial de dois operadores $\hat{F}$ e $\hat{G}$, dados por matrizes $2 \times 2$, é determinado pelo produto de Kronecker:

$$
\begin{aligned}
& \hat{F} \otimes \hat{G}=\left(\begin{array}{ll}
F_{11} & F_{12} \\
F_{21} & F_{22}
\end{array}\right) \otimes\left(\begin{array}{ll}
G_{11} & G_{12} \\
G_{21} & G_{22}
\end{array}\right)= \\
& \left.=\left(\begin{array}{cc}
F_{11}\left(\begin{array}{ll}
G_{11} & G_{12} \\
G_{21} & G_{22}
\end{array}\right) & F_{12}\left(\begin{array}{ll}
G_{11} & G_{12} \\
G_{21} & G_{22} \\
G_{11} & G_{12} \\
G_{21} & G_{22}
\end{array}\right) \\
G_{11} & G_{12} \\
G_{21} & G_{22}
\end{array}\right)\right) .
\end{aligned}
$$

Vamos construir o operador $\sigma_{z} \otimes \sigma_{z}$,

$$
\sigma_{z} \otimes \sigma_{z}=\left(\begin{array}{cc}
1 & 0 \\
0 & -1
\end{array}\right) \otimes\left(\begin{array}{cc}
1 & 0 \\
0 & -1
\end{array}\right)=\left(\begin{array}{cccc}
1 & 0 & 0 & 0 \\
0 & -1 & 0 & 0 \\
0 & 0 & -1 & 0 \\
0 & 0 & 0 & 1
\end{array}\right) .
$$

Podemos ver que os vetores da base computacional são auto-estados do operador $\sigma_{z} \otimes \sigma_{z}$,

$$
\begin{aligned}
& \sigma_{z} \otimes \sigma_{z}|01\rangle=-|01\rangle, \sigma_{z} \otimes \sigma_{z}|10\rangle=-|10\rangle, \\
& \sigma_{z} \otimes \sigma_{z}|00\rangle=|00\rangle, \sigma_{z} \otimes \sigma_{z}|11\rangle=|11\rangle .
\end{aligned}
$$


Para completar, construímos os seguintes operadores de densidade

$$
\begin{aligned}
|00\rangle\langle 00| & =\left(\begin{array}{llll}
1 & 0 & 0 & 0 \\
0 & 0 & 0 & 0 \\
0 & 0 & 0 & 0 \\
0 & 0 & 0 & 0
\end{array}\right),|00\rangle\langle 11|=\left(\begin{array}{llll}
0 & 0 & 0 & 1 \\
0 & 0 & 0 & 0 \\
0 & 0 & 0 & 0 \\
0 & 0 & 0 & 0
\end{array}\right), \\
|11\rangle\langle 00| & =\left(\begin{array}{llll}
0 & 0 & 0 & 0 \\
0 & 0 & 0 & 0 \\
0 & 0 & 0 & 0 \\
1 & 0 & 0 & 0
\end{array}\right),|11\rangle\langle 11|=\left(\begin{array}{llll}
0 & 0 & 0 & 0 \\
0 & 0 & 0 & 0 \\
0 & 0 & 0 & 0 \\
0 & 0 & 0 & 1
\end{array}\right) .
\end{aligned}
$$

e

$$
\begin{aligned}
& |01\rangle\left\langle 00\left|=\left(\begin{array}{llll}
0 & 0 & 0 & 0 \\
1 & 0 & 0 & 0 \\
0 & 0 & 0 & 0 \\
0 & 0 & 0 & 0
\end{array}\right), \quad\right| 01\right\rangle\langle 11|=\left(\begin{array}{llll}
0 & 0 & 0 & 0 \\
0 & 0 & 0 & 0 \\
0 & 0 & 0 & 1 \\
0 & 0 & 0 & 0
\end{array}\right), \\
& |10\rangle\left\langle 01\left|=\left(\begin{array}{llll}
0 & 0 & 0 & 0 \\
0 & 1 & 0 & 0 \\
0 & 0 & 0 & 0 \\
0 & 0 & 0 & 0
\end{array}\right), \quad\right| 10\right\rangle\langle 10|=\left(\begin{array}{llll}
0 & 0 & 0 & 0 \\
0 & 0 & 0 & 0 \\
0 & 0 & 1 & 0 \\
0 & 0 & 0 & 0
\end{array}\right),
\end{aligned}
$$

O exemplo mais famoso de um estado emaranhado de duas partículas (ver abaixo) é o proposto por Bohm [2], um estado emaranhado de dois spins $1 / 2$,

$$
\left|\Psi^{-}\right\rangle=\frac{1}{\sqrt{2}}[|\uparrow\rangle \otimes|\downarrow\rangle-|\downarrow\rangle \otimes|\uparrow\rangle]=\frac{1}{\sqrt{2}}[|01\rangle-|10\rangle] .
$$

Esse estado, juntamente com três outros estados formam a chamada base de Bell [7],

$$
\begin{aligned}
& \left|\Phi^{ \pm}\right\rangle=\frac{1}{\sqrt{2}}[|\uparrow\rangle \otimes|\uparrow\rangle \pm|\downarrow\rangle \otimes|\downarrow\rangle]=\frac{1}{\sqrt{2}}[|00\rangle \pm|11\rangle], \\
& \left|\Psi^{ \pm}\right\rangle=\frac{1}{\sqrt{2}}[|\uparrow\rangle \otimes|\downarrow\rangle \pm|\downarrow\rangle \otimes|\uparrow\rangle]=\frac{1}{\sqrt{2}}[|01\rangle \pm|10\rangle] .
\end{aligned}
$$

Esses estados não podem ser escritos como um produto tensorial simples de dois estados que pertencem a dois subsistemas separadamente. Eles são estados emaranhados. 
A chamada base mágica que é utilizada para calcular a medida de emaranhamento está relacionada com a base de Bell (3.38), de acordo com a qual

$$
\begin{aligned}
& \left|e_{1}\right\rangle=\frac{1}{\sqrt{2}}[|00\rangle+|11\rangle]=\left|\Phi^{+}\right\rangle,\left|e_{2}\right\rangle=\frac{i}{\sqrt{2}}[|00\rangle-|11\rangle]=i\left|\Phi^{-}\right\rangle, \\
& \left|e_{3}\right\rangle=\frac{i}{\sqrt{2}}[|01\rangle+|10\rangle]=i\left|\Psi^{+}\right\rangle,\left|e_{4}\right\rangle=\frac{1}{\sqrt{2}}[|01\rangle-|10\rangle]=\left|\Psi^{-}\right\rangle .
\end{aligned}
$$

Considere um caso particular dos estados emaranhados:

$$
|\Psi\rangle=\vartheta_{00}|00\rangle+\vartheta_{11}|11\rangle, \quad\left|\vartheta_{00}\right|^{2}+\left|\vartheta_{11}\right|^{2}=1 .
$$

Primeiro, vamos calcular o operador densidade correspondente ao estado (3.40),

$$
\begin{aligned}
\hat{\rho} & =|\Psi\rangle\left\langle\Psi \left|=\left(\vartheta_{00}|00\rangle+\vartheta_{11}|11\rangle\right)\left(\vartheta_{00}^{*}\langle 00|+\vartheta_{11}^{*}\langle 11|\right)\right.\right. \\
& =\left|\vartheta_{00}\right|^{2}|00\rangle\left\langle 00\left|+\vartheta_{00} \vartheta_{11}^{*}\right| 00\right\rangle\left\langle 11\left|+\vartheta_{00}^{*} \vartheta_{11}\right| 11\right\rangle\left\langle\left. 00|+| \vartheta_{11}\right|^{2} \mid 11\right\rangle\langle 11| .
\end{aligned}
$$

Usando eqs. (3.35) e (3.36), obtemos

$$
\hat{\rho}=\left(\begin{array}{cccc}
\left|\vartheta_{00}\right|^{2} & 0 & 0 & u v^{*} \\
0 & 0 & 0 & 0 \\
0 & 0 & 0 & 0 \\
v u^{*} & 0 & 0 & \left|\vartheta_{11}\right|^{2}
\end{array}\right) .
$$

Agora, estamos em posição para obter os operadores estatísticos reduzidos de ambos os subsistemas (veja 3.32). Temos

$$
\begin{aligned}
& \hat{\rho}_{A}=\operatorname{tr}_{B} \hat{\rho}={ }_{B}\langle 0|\hat{\rho}| 0\rangle_{B}+{ }_{B}\langle 1|\hat{\rho}| 1\rangle_{B} \\
& =\left|\vartheta_{00}\right|^{2}|0\rangle\left\langle\left. 0|+| \vartheta_{11}\right|^{2} \mid 1\right\rangle\langle 1| .
\end{aligned}
$$

Da mesma maneira, obtemos

$$
\begin{aligned}
& \hat{\rho}_{B}=\operatorname{tr}_{A} \hat{\rho}={ }_{A}\langle 0|\hat{\rho}| 0\rangle_{A}+{ }_{A}\langle 1|\hat{\rho}| 1\rangle_{A} \\
& \quad=\left|\vartheta_{00}\right|^{2}|0\rangle\left\langle\left. 0|+| \vartheta_{11}\right|^{2} \mid 1\right\rangle\langle 1| .
\end{aligned}
$$

Assim, ambos operadores estatísticos reduzidos são iguais, $\hat{\rho}_{A}=\hat{\rho}_{B}$. Levando em conta Eq. (3.32), podemos escrever,

$$
\hat{\rho}_{A}=\hat{\rho}_{B}=\left(\begin{array}{cc}
\left|\vartheta_{00}\right|^{2} & 0 \\
0 & \left|\vartheta_{11}\right|^{2}
\end{array}\right),
$$


tal que ambos operadores estatísticos reduzidos têm dois autovalores $\lambda_{1}=$ $\left|\vartheta_{00}\right|^{2}$ e $\lambda_{2}=\left|\vartheta_{11}\right|^{2}$.

Vamos considerar o estado puro geral

$$
|\Psi\rangle=v_{1}|00\rangle+v_{4}|11\rangle+v_{2}|01\rangle+v_{3}|10\rangle .
$$

O operador estatístico correspondente tem a forma

$$
\begin{gathered}
\hat{\rho}=|\Psi\rangle\left\langle\Psi \left|=\left[v_{1}|00\rangle+v_{2}|01\rangle+v_{3}|10\rangle+v_{4}|11\rangle\right]\right.\right. \\
{\left[v_{1}^{*}\langle 00|+v_{2}^{*}\langle 01|+v_{3}^{*}\langle 10|+v_{4}^{*}\langle 11|\right]}
\end{gathered}
$$

Calculando o operador estatístico reduzido do subsistema $A$, obtemos

$$
\begin{aligned}
& \hat{\rho}_{A}=\operatorname{tr}_{B} \hat{\rho}={ }_{B}\langle 0|\hat{\rho}| 0\rangle_{B}+{ }_{B}\langle 1|\hat{\rho}| 1\rangle_{B} \\
& =\left|v_{1}\right|^{2}|0\rangle\left\langle 0\left|+v_{1} v_{3}^{*}\right| 0\right\rangle\left\langle\left. 1|+| v_{2}\right|^{2} \mid 0\right\rangle\left\langle 0\left|+v_{2} v_{4}^{*}\right| 0\right\rangle\langle 1| \\
& +v_{3} v_{1}^{*}|1\rangle\left\langle\left. 0|+| v_{3}\right|^{2} \mid 1\right\rangle\left\langle 1\left|+v_{4} v_{2}^{*}\right| 1\right\rangle\left\langle\left. 0|+| v_{4}\right|^{2} \mid 1\right\rangle\langle 1| \\
& =\left(\begin{array}{cc}
\rho_{11}^{(A)} & \rho_{12}^{(A)} \\
\rho_{21}^{(A)} & \rho_{22}^{(A)}
\end{array}\right),
\end{aligned}
$$

onde

$$
\begin{aligned}
& \rho_{11}^{(A)}=\left|v_{1}\right|^{2}+\left|v_{2}\right|^{2}, \quad \rho_{12}^{(A)}=v_{1} v_{3}^{*}+v_{2} v_{4}^{*}, \\
& \rho_{21}^{(A)}=v_{3} v_{1}^{*}+v_{4} v_{2}^{*}, \quad \rho_{22}^{(A)}=\left|v_{3}\right|^{2}+\left|v_{4}\right|^{2} .
\end{aligned}
$$

\subsection{Sistemas de três qubits}

Um sistema quântico se chama tri-partido se ele consiste em dois subsistemas $A, B$, e $C$, e seu espaço de Hilbert é

$$
\mathcal{H}=\mathcal{H}_{A} \otimes \mathcal{H}_{B} \otimes \mathcal{H}_{C}=\mathbb{C}^{2} \otimes \mathbb{C}^{2} \otimes \mathbb{C}^{2}
$$

Sejam $\left\{|a\rangle_{A}, a=1,2\right\}$ e bases ortonormal em espaço $\mathcal{H}_{A},\left\{|b\rangle_{B}, b=1,2\right\}$ e bases ortonormal em espaço $\mathcal{H}_{B}$, e $\left\{|c\rangle_{C}, c=1,2\right\}$ e bases ortonormal em espaço $\mathcal{H}_{C}$

$$
{ }_{A}\left\langle a \mid a^{\prime}\right\rangle_{A}=\delta_{a a^{\prime}}, \quad{ }_{B}\left\langle b \mid b^{\prime}\right\rangle_{B}=\delta_{b b^{\prime}}, \quad{ }_{C}\left\langle c \mid c^{\prime}\right\rangle_{C}=\delta_{c c^{\prime}} .
$$

Nesse caso, $\{|a, b, c\rangle=|a\rangle \otimes|b\rangle \otimes|c\rangle\}$ é uma base no espaço total $\mathcal{H}$, 
Qualquer estado puro $|\Psi\rangle \in \mathcal{H}$ pode ser decomposto nessa base como

$$
|\Psi\rangle=\sum_{a, b, c=1}^{2} \vartheta_{a b c}|a\rangle \otimes|b\rangle \otimes|c\rangle,
$$

onde $\vartheta_{a b c}$ são alguns coeficientes.

Primeiramente, lembremos algumas noções do produto tensorial de espaços vetoriais tendo em mente nossos três espaços 2-dimensional $\mathbb{C}^{2}$ e 8dimensional $\mathbb{C}^{2} \otimes \mathbb{C}^{2} \otimes \mathbb{C}^{2}$. Considere três vetores de base

$$
|a\rangle=\left(\begin{array}{l}
a_{1} \\
a_{2}
\end{array}\right), \quad|b\rangle=\left(\begin{array}{l}
b_{1} \\
b_{2}
\end{array}\right),|c\rangle=\left(\begin{array}{c}
c_{1} \\
c_{2}
\end{array}\right) .
$$

Então,

$$
\begin{aligned}
& |a\rangle \otimes|b\rangle \otimes|c\rangle=\left(\begin{array}{l}
a_{1} \\
a_{2}
\end{array}\right) \otimes\left(\begin{array}{l}
b_{1} \\
b_{2}
\end{array}\right) \otimes\left(\begin{array}{l}
c_{1} \\
c_{2}
\end{array}\right) \\
& =\left(\begin{array}{ll}
a_{1} & \left(\begin{array}{l}
b_{1} \\
b_{2}
\end{array}\right) \\
a_{2} & \left(\begin{array}{l}
b_{1} \\
b_{2}
\end{array}\right)
\end{array}\right) \otimes\left(\begin{array}{l}
c_{1} \\
c_{2}
\end{array}\right) \\
& =\left(\begin{array}{l}
a_{1} b_{1} c_{1} \\
a_{1} b_{1} c_{2} \\
a_{1} b_{2} c_{1} \\
a_{2} b_{1} \\
a_{2} b_{2}
\end{array}\right) \otimes\left(\begin{array}{l}
c_{1} \\
c_{2} b_{2} c_{2} \\
a_{2} b_{1} c_{1} \\
a_{2} b_{1} c_{2} \\
a_{2} b_{2} c_{1} \\
a_{2} b_{2} c_{2}
\end{array}\right) .
\end{aligned}
$$


A base computacional $\left|\Theta_{i}\right\rangle, i=1, \ldots, 8$, neste caso, definimos como

$$
\begin{aligned}
& \left|\Theta_{1}\right\rangle=|000\rangle=\left(\begin{array}{l}
1 \\
0
\end{array}\right) \otimes\left(\begin{array}{l}
1 \\
0
\end{array}\right) \otimes\left(\begin{array}{l}
1 \\
0
\end{array}\right)=\left(\begin{array}{cccccccc}
1 & 0 & 0 & 0 & 0 & 0 & 0 & 0
\end{array}\right)^{T} ; \\
& \left|\Theta_{2}\right\rangle=|001\rangle=\left(\begin{array}{l}
1 \\
0
\end{array}\right) \otimes\left(\begin{array}{l}
1 \\
0
\end{array}\right) \otimes\left(\begin{array}{l}
0 \\
1
\end{array}\right)=\left(\begin{array}{llllllll}
0 & 1 & 0 & 0 & 0 & 0 & 0 & 0
\end{array}\right)^{T} ; \\
& \left|\Theta_{3}\right\rangle=|010\rangle=\left(\begin{array}{l}
1 \\
0
\end{array}\right) \otimes\left(\begin{array}{l}
0 \\
1
\end{array}\right) \otimes\left(\begin{array}{l}
1 \\
0
\end{array}\right)=\left(\begin{array}{cccccccc}
0 & 0 & 1 & 0 & 0 & 0 & 0 & 0
\end{array}\right)^{T} \text {; } \\
& \left|\Theta_{4}\right\rangle=|011\rangle=\left(\begin{array}{l}
1 \\
0
\end{array}\right) \otimes\left(\begin{array}{l}
0 \\
1
\end{array}\right) \otimes\left(\begin{array}{l}
0 \\
1
\end{array}\right)=\left(\begin{array}{llllllll}
0 & 0 & 0 & 1 & 0 & 0 & 0 & 0
\end{array}\right)^{T} \text {; } \\
& \left|\Theta_{5}\right\rangle=|100\rangle=\left(\begin{array}{l}
0 \\
1
\end{array}\right) \otimes\left(\begin{array}{l}
1 \\
0
\end{array}\right) \otimes\left(\begin{array}{l}
1 \\
0
\end{array}\right)=\left(\begin{array}{llllllll}
0 & 0 & 0 & 0 & 1 & 0 & 0 & 0
\end{array}\right)^{T} \text {; } \\
& \left|\Theta_{6}\right\rangle=|101\rangle=\left(\begin{array}{l}
0 \\
1
\end{array}\right) \otimes\left(\begin{array}{l}
1 \\
0
\end{array}\right) \otimes\left(\begin{array}{l}
0 \\
1
\end{array}\right)=\left(\begin{array}{cccccccc}
0 & 0 & 0 & 0 & 0 & 1 & 0 & 0
\end{array}\right)^{T} \text {; } \\
& \left|\Theta_{7}\right\rangle=|110\rangle=\left(\begin{array}{l}
0 \\
1
\end{array}\right) \otimes\left(\begin{array}{l}
0 \\
1
\end{array}\right) \otimes\left(\begin{array}{l}
1 \\
0
\end{array}\right)=\left(\begin{array}{llllllll}
0 & 0 & 0 & 0 & 0 & 0 & 1 & 0
\end{array}\right)^{T} \text {; } \\
& \left|\Theta_{8}\right\rangle=|111\rangle=\left(\begin{array}{l}
0 \\
1
\end{array}\right) \otimes\left(\begin{array}{l}
0 \\
1
\end{array}\right) \otimes\left(\begin{array}{l}
0 \\
1
\end{array}\right)=\left(\begin{array}{llllllll}
0 & 0 & 0 & 0 & 0 & 0 & 0 & 1
\end{array}\right)^{T} .
\end{aligned}
$$

Um vetor arbitrário $|\Psi\rangle$ na base computacional tem a seguinte forma

$$
|\Psi\rangle=\sum_{i, j, k=0,1} \vartheta_{i j k}|i j k\rangle=\sum_{s=1}^{8} v_{s}\left|\Theta_{s}\right\rangle .
$$

Considerando a equação anterior, um operador estatístico que corresponde a um estado puro arbitrário (3.55) pode ser escrito como

$$
\begin{aligned}
& \hat{\rho}=\left(\sum_{i, j, k} \vartheta_{i j k}|i j k\rangle\right)\left(\sum_{i, j, k} \vartheta_{i j k}^{*}\langle i j k|\right) \\
= & \left(\sum_{s=1}^{8} v_{s}\left|\Theta_{s}\right\rangle\right)\left(\sum_{r=1}^{8} v_{r}^{*}\left\langle\Theta_{r}\right|\right) \\
= & \left(\vartheta_{000}|000\rangle+\cdots+\vartheta_{111}|111\rangle\right)\left(\vartheta_{000}^{*}\langle 000|+\cdots+\vartheta_{111}^{*}\langle 111|\right) \\
= & \left(v_{1}\left|\Theta_{1}\right\rangle+\cdots+v_{8}\left|\Theta_{8}\right\rangle\right)\left(v_{1}^{*}\left\langle\Theta_{1}\right|+\cdots+v_{8}^{*}\left\langle\Theta_{8}\right|\right) .
\end{aligned}
$$


Vamos calcular a matriz de densidade reduzida $\hat{\rho}^{(C)}$,

$$
\begin{aligned}
& \hat{\rho}^{(C)}=\operatorname{tr}_{A B} \hat{\rho}=\sum_{a, b=0,1}\langle a, b|\hat{\rho}| a, b\rangle \\
& =\left\langle 0_{A}, 0_{B}|\hat{\rho}| 0_{A}, 0_{B}\right\rangle+\left\langle 1_{A}, 0_{B}|\hat{\rho}| 1_{A}, 0_{B}\right\rangle+\left\langle 1_{A}, 1_{B}|\hat{\rho}| 1_{A}, 1_{B}\right\rangle \\
& +\left\langle 0_{A}, 1_{B}|\hat{\rho}| 0_{A}, 1_{B}\right\rangle=\left(\left|\vartheta_{000}\right|^{2}+\left|\vartheta_{100}\right|^{2}+\left|\vartheta_{110}\right|^{2}+\left|\vartheta_{010}\right|^{2}\right) \\
& \times|0\rangle\left\langle 0\left|+\left(\vartheta_{000} \vartheta_{001}^{*}+\vartheta_{100} \vartheta_{101}^{*}+\vartheta_{010} \vartheta_{011}^{*}+\vartheta_{010} \vartheta_{011}^{*}\right)\right| 0\right\rangle\langle 1| \\
& +\left(\vartheta_{001} \vartheta_{000}^{*}+\vartheta_{101} \vartheta_{100}^{*}+\vartheta_{111} \vartheta_{110}^{*}+\vartheta_{011} \vartheta_{010}^{*}\right)|1\rangle\langle 0| \\
& +\left(\left|\vartheta_{001}\right|^{2}+\left|\vartheta_{101}\right|^{2}+\left|\vartheta_{111}\right|^{2}+\left|\vartheta_{011}\right|^{2}\right)|1\rangle\langle 1|| 1\rangle\langle 0| \\
& =\left|v_{1}\right|^{2}|0\rangle\left\langle 0\left|+v_{1} v_{2}^{*}\right| 0\right\rangle\left\langle 1\left|+v_{2} v_{1}^{*}\right| 1\right\rangle\left\langle\left. 0|+| v_{2}\right|^{2} \mid 1\right\rangle\langle 1| \\
& +v_{3} v_{3}^{*}|0\rangle\left\langle 0\left|+v_{3} v_{4}^{*}\right| 0\right\rangle\left\langle 1\left|+v_{4} v_{3}^{*}\right| 1\right\rangle\left\langle\left. 0|+| v_{4}\right|^{2} \mid 1\right\rangle\langle 1| \\
& +v_{5} v_{5}^{*}|0\rangle\left\langle 0\left|+v_{5} v_{6}^{*}\right| 0\right\rangle\left\langle 1\left|+v_{6} v_{5}^{*}\right| 1\right\rangle\left\langle\left. 0|+| v_{6}\right|^{2} \mid 1\right\rangle\langle 1| \\
& +v_{7} v_{7}^{*}|0\rangle\left\langle 0\left|+v_{7} v_{8}^{*}\right| 0\right\rangle\left\langle 1\left|+v_{8} v_{7}^{*}\right| 1\right\rangle\left\langle\left. 0|+| v_{8}\right|^{2} \mid 1\right\rangle\langle 1| .
\end{aligned}
$$

Tomando em conta a Eq. (3.32), obtemos

$$
\hat{\rho}^{(C)}=\left(\begin{array}{ll}
\rho_{11}^{(C)} & \rho_{12}^{(C)} \\
\rho_{21}^{(C)} & \rho_{22}^{(C)}
\end{array}\right),
$$

onde

$$
\begin{aligned}
\rho_{11}^{(C)} & =\left|\vartheta_{000}\right|^{2}+\left|\vartheta_{100}\right|^{2}+\left|\vartheta_{110}\right|^{2}+\left|\vartheta_{010}\right|^{2}=\left|v_{1}\right|^{2}+\left|v_{3}\right|^{2}+\left|v_{5}\right|^{2}+\left|v_{7}\right|^{2}, \\
\rho_{12}^{(C)} & =\vartheta_{000} \vartheta_{001}^{*}+\vartheta_{100} \vartheta_{101}^{*}+\vartheta_{010} \vartheta_{011}^{*}+\vartheta_{010} \vartheta_{011}^{*}=v_{1} v_{2}^{*}+v_{3} v_{4}^{*}+v_{5} v_{6}^{*}+v_{7} v_{8}^{*}, \\
\rho_{21}^{(C)} & =\vartheta_{001} \vartheta_{000}^{*}+\vartheta_{101} \vartheta_{100}^{*}+\vartheta_{111} \vartheta_{110}^{*}+\vartheta_{011} \vartheta_{010}^{*}=v_{1}^{*} v_{2}+v_{3}^{*} v_{4}+v_{5}^{*} v_{6}+v_{7}^{*} v_{8}, \\
\rho_{22}^{(C)} & =\left|\vartheta_{001}\right|^{2}+\left|\vartheta_{101}\right|^{2}+\left|\vartheta_{111}\right|^{2}+\left|\vartheta_{011}\right|^{2}=\left|v_{2}\right|^{2}+\left|v_{4}\right|^{2}+\left|v_{6}\right|^{2}+\left|v_{8}\right|^{2} .(3.59)
\end{aligned}
$$

\subsection{Medidas do emaranhamento}

\subsubsection{Testemunhas de emaranhamento}

Testemunhas de emaranhamento são observáveis (operadores) que nos permitem detectar o emaranhamento. Existe o seguinte teorema dos Horodeckis [43].

A matriz de densidade $\hat{\rho}$ é emaranhada se, e somente se, existe um operador hermitiano $\hat{W}$ com as propriedades 
- $\operatorname{Tr}(\hat{W} \hat{\rho})<0$

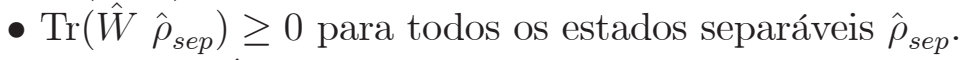

O operador $\hat{W}$ é chamado de testemunha de emaranhamento.

\subsubsection{Medida de emaranhamento}

É útil quantificar a quantidade de emaranhamento contida em um determinado estado. Portanto, temos de definir uma medida de emaranhamento apropriada. Assumimos um sistema bipartido com espaço de Hilbert $\mathcal{H}=$ $\mathcal{H}_{A} \otimes \mathcal{H}_{B}, \mathrm{e} \operatorname{dim} \mathcal{H}_{A}=\operatorname{dim} \mathcal{H}_{B}=d$. Então: Uma medida de emaranhamento é uma função $E(\rho)$ que atribui a cada estado $\hat{\rho}$ de um sistema bipartido dimensional finito um número real positivo $E(\rho) \in \mathbb{R}_{+}$. A medida de emaranhamento $E(\rho)$ desaparece para estados separáveis, $E\left(\rho_{\text {sep }}\right)=0$, e assume o seu máximo em estados maximalmente emaranhados $E\left(\rho_{M E}\right)=\log _{2}(d)$. Se $\mathcal{H}=\mathcal{H}_{A} \otimes \mathcal{H}_{B}=\mathbb{C}^{2} \otimes \mathbb{C}^{2}$, então $E\left(\rho_{M E}\right)=1$.

A entropia de von Neumann de um estado $\hat{\rho}$, definida por Eg. (3.11), pode ser vista como uma "quantização"direta da entropia clássica de Shannon. Um tipo da medida de emaranhamento foi oferecido por Charles H. Bennet e coautores, em 1996, no trabalho [44]. Uma medida emaranhada $E(\Psi)$ de um estado puro $|\Psi\rangle$ de um sistema bipartido é definida da seguinte forma:

$$
E(\Psi)=S\left(\rho^{(A)}\right)=S\left(\rho^{(B)}\right),
$$

onde $S\left(\rho_{A}\right)$ é a entropia de von Neumann do operador estatístico reduzido de um subsistema $A$ ou $B$ (pode-se verificar que eles são iguais). Usando a definição (3.11), obtemos

$$
E(\Psi)=-\operatorname{tr}\left(\hat{\rho}^{(A)} \log \hat{\rho}^{(A)}\right)=-\operatorname{tr}\left(\hat{\rho}^{(B)} \log \hat{\rho}^{(B)}\right) .
$$

No caso de um estado puro emaranhado, seus operadores estatísticos reduzidos têm entropia quântica diferente de zero, ao passo que a entropia do estado puro inicial é sempre zero.

Pela definição, um estado puro $|\Psi\rangle$ de um sistema bipartido é maximalmente emaranhado se seus operadores estatísticos reduzidos são proporcionais ao operador-identidade.

A entropia de von Neumann satisfaz todas as propriedades anteriormente mencionadas e é o único candidato para uma medida de emaranhamento que é fisicamente razoável. 
Vamos encontrar a entropia de von Neumann dos operadores estatísticos reduzidos (3.43) usando eqs. (3.61),

$$
E(\Psi)=-\operatorname{tr}\left(\hat{\rho}^{(A)} \ln \hat{\rho}^{(A)}\right)=-\operatorname{tr}\left(\hat{\rho}^{(B)} \ln \hat{\rho}^{(B)}\right) .
$$

De (3.43) resulta

$$
\ln \hat{\rho}^{(A)}=\left(\begin{array}{cc}
\ln \lambda_{1} & 0 \\
0 & \ln \lambda_{2}
\end{array}\right)
$$

Então,

$$
\hat{\rho}^{(A)} \ln \hat{\rho}^{(A)}=\left(\begin{array}{cc}
\lambda_{1} & 0 \\
0 & \lambda_{2}
\end{array}\right)\left(\begin{array}{cc}
\ln \lambda_{1} & 0 \\
0 & \ln \lambda_{2}
\end{array}\right)=\left(\begin{array}{cc}
\lambda_{1} \ln \lambda_{1} & 0 \\
0 & \lambda_{2} \ln \lambda_{2}
\end{array}\right) .
$$

Finalmente,

$$
\begin{aligned}
E(\Psi) & =-\operatorname{tr}\left(\hat{\rho}^{(A)} \ln \hat{\rho}^{(A)}\right)=-\sum_{i=1,2} \lambda_{i} \ln \lambda_{i} \\
& =-\left(\left|\vartheta_{11}\right|^{2} \ln \left|\vartheta_{11}\right|^{2}+\left|\vartheta_{22}\right|^{2} \ln \left|\vartheta_{22}\right|^{2}\right) .
\end{aligned}
$$

Uma vez que $\lambda_{1}+\lambda_{2}=\left|\vartheta_{11}\right|^{2}+\left|\vartheta_{22}\right|^{2}=1 \neq 0$, a medida do emaranhamento $E(\Psi)$ não é igual a zero. E é igualmente positivo, pois ambos $\lambda_{1}$ e $\lambda_{2}$ são inferiores a um $\left(\lambda_{1}, \lambda_{2} \leq 1\right)$.

O emaranhamento é máximo se $\left|\vartheta_{11}\right|^{2}=\left|\vartheta_{22}\right|^{2}=1 / 2$.

\subsubsection{O cálculo de emaranhamento de sistema de dois qubits em caso geral}

Vamos calcular a medida do emaranhamento (3.61) usando expressões explicitas para operadores estatísticos reduzidos dos estados puros de um sistema bipartido dados por Eqs. (3.47) e (3.48).

É possível encontrar os autovalores $\lambda_{1}$ e $\lambda_{2}$, e os autovetores $P_{1}$ e $P_{2}$ da 
matriz (3.47). O resultado é:

$$
\begin{aligned}
& \hat{\rho}^{(A)} P_{i}=\lambda_{i} P_{i}, \quad i=1,2, \\
& \lambda_{1}=\frac{1}{2}\left[\rho_{11}^{(A)}-\sqrt{4 \rho_{12}^{(A)} \rho_{21}^{(A)}+\left(\rho_{11}^{(A)}-\rho_{22}^{(A)}\right)^{2}}+\rho_{22}^{(A)}\right], \\
& \lambda_{2}=\frac{1}{2}\left[\rho_{11}^{(A)}+\sqrt{4 \rho_{12}^{(A)} \rho_{21}^{(A)}+\left(\rho_{11}^{(A)}-\rho_{22}^{(A)}\right)^{2}}+\rho_{22}^{(A)}\right] \\
& P_{1}=\left(\begin{array}{c}
\left.-\frac{-\rho_{11}^{(A)}+\sqrt{4 \rho_{12}^{(A)} \rho_{21}^{(A)}+\left(\rho_{11}^{(A)}-\rho_{22}^{(A)}\right)^{2}}+\rho_{22}^{(A)}}{2 \rho_{21}^{(A)}}\right) \\
1
\end{array}\right) \\
& P_{2}=\left(\begin{array}{c}
\frac{\rho_{11}^{(A)}+\sqrt{4 \rho_{12}^{(A)} \rho_{21}^{(A)}+\left(\rho_{11}^{(A)}-\rho_{22}^{(A)}\right)^{2}}-\rho_{22}^{(A)}}{2 \rho_{21}^{(A)}} \\
1
\end{array}\right) .
\end{aligned}
$$

Uma simples verificação mostra

$$
\operatorname{tr} \hat{\rho}^{(A)}=\rho_{11}^{(A)}+\rho_{22}^{(A)}=\lambda_{1}+\lambda_{2}
$$

Assim, encontramos

$$
\begin{aligned}
& E(\Psi)=-\operatorname{tr}\left(\hat{\rho}^{(A)} \log _{2} \hat{\rho}^{(A)}\right)=-\sum_{i=1,2} \lambda_{i} \log _{2} \lambda_{i} \\
& =-\left[z \log _{2} z+(1-z) \log _{2}(1-z)\right]
\end{aligned}
$$

onde

$$
z=\frac{1+y}{2}, y=\sqrt{\left(\rho_{11}^{(A)}-\rho_{22}^{(A)}\right)^{2}+4\left|\rho_{12}^{(A)}\right|^{2}} .
$$

Expressando os elementos da matriz de densidade $\rho_{a b}^{(C)}$ via coeficientes $v_{i}, i=$ $1, \ldots, 8$ de acordo com Eqs. (3.56) e (3.57), obtemos

$$
y=\sqrt{\left(\left|v_{1}\right|^{2}+\left|v_{2}\right|^{2}-\left|v_{4}\right|^{2}-\left|v_{3}\right|^{2}\right)^{2}+4\left|v_{1} v_{3}^{*}+v_{2} v_{4}^{*}\right|^{2}} .
$$

Uma forma útil e construtiva para calcular a medida do emaranhamento $E(\Psi)$, dada pela equação $(3.61)$, foi proposta no trabalho $[46,47]$. Ela é expressa por meio da chamada concorrência $C(\Psi)$,

$$
C(\Psi)=\left|\sum_{k} \alpha_{k}^{2}\right|,
$$


onde $\alpha_{k}$ são os coeficientes da decomposição do vetor de estado $|\Psi\rangle$ de um sistema bipartido com respeito à chamada base mágica $\left|e_{k}\right\rangle, k=1,2,3,4$, dada pelas equações (3.39),

$$
|\Psi\rangle=\sum_{k=1}^{4} \alpha_{k}\left|e_{k}\right\rangle
$$

Deve-se notar que, no caso geral, os coeficientes $\alpha_{k}$ são números complexos e entram na medida (3.69), ou seja, como números complexos, mas não o seu módulo. Pode-se verificar que cada vetor da base de Bell e da base mágica são emaranhados maximalmente. Dessa maneira, a quantidade de concorrência $C(\Psi)$ mostra como um estado puro $|\Psi\rangle$ pode ser aproximado por uma mistura de estados emaranhados maximalmente.

Assim, a medida de emaranhamento $E(\Psi)$ é (detalhes desta prova podem ser encontrados na Ref. [46, 47])

$$
E(\Psi)=H(x), \operatorname{com} x=\frac{1}{2}\left(1+\sqrt{1-C^{2}}\right) .
$$

Aqui, $H(x)$ é a chamada função entropia binária,

$$
H(x)=-x \log _{2}(x)-(1-x) \log _{2}(1-x) .
$$

Deve-se notar que existem outras características quantitativas da medida de emaranhamento, veja Refs. [48, 49, 50, 51, 52, 53, 54, 55].

\subsubsection{O emaranhamento do estado puro de três qubits em caso geral}

Aqui, vamos seguir o trabalho [56] na definição de uma medida de emaranhamento para o estado puro de três qubits. Seja

$$
|\Psi\rangle=\sum_{i, j, k=0,1} \vartheta_{i j k}|i j k\rangle=\sum_{s=1}^{8} v_{s}\left|\Theta_{s}\right\rangle .
$$

e um estado puro, veja Eqs. (3.54) e (3.55).

De acordo com artigo [56], vamos definir o chamado emaranhamento residual $\tau_{A B C}$ como a medida de emaranhamento para o estado puro de 
três qubits,

$$
\begin{aligned}
& \tau_{A B C}=2\left|\sum \vartheta_{i j k} \vartheta_{i^{\prime} j^{\prime} m} \vartheta_{n p k^{\prime}} \vartheta_{n^{\prime} p^{\prime} m^{\prime}} \epsilon_{i i^{\prime}} \epsilon_{j j^{\prime}} \epsilon_{k k^{\prime}} \epsilon_{m m^{\prime}} \epsilon_{n n^{\prime}} \epsilon_{p p^{\prime}}\right| \\
& =4\left|d_{1}-2 d_{2}+4 d_{3}\right|,
\end{aligned}
$$

em que a primeira soma vai sobre todos os índices e onde

$$
\begin{aligned}
& d_{1}=\vartheta_{000}^{2} \vartheta_{111}^{2}+\vartheta_{001}^{2} \vartheta_{110}^{2}+\vartheta_{010}^{2} \vartheta_{101}^{2}+\vartheta_{100}^{2} \vartheta_{011}^{2} ; \\
& d_{2}=\vartheta_{000} \vartheta_{111} \vartheta_{011} \vartheta_{100}+\vartheta_{000} \vartheta_{111} \vartheta_{101} \vartheta_{010} \\
& +\vartheta_{000} \vartheta_{111} \vartheta_{110} \vartheta_{001}+\vartheta_{011} \vartheta_{100} \vartheta_{101} \vartheta_{010} \\
& +\vartheta_{011} \vartheta_{100} \vartheta_{110} \vartheta_{001}+\vartheta_{101} \vartheta_{010} \vartheta_{110} \vartheta_{001} \\
& d_{3}=\vartheta_{000} \vartheta_{110} \vartheta_{101} \vartheta_{011}+\vartheta_{111} \vartheta_{001} \vartheta_{010} \vartheta_{100} .
\end{aligned}
$$

É possível provar que o emaranhamento residual é invariante sobre permutação de todos os qubits,

$$
\tau_{A B C}=\tau_{B A C}=\cdots=\tau_{C B A} .
$$

Tentamos também, no caso considerado, usar a entropia de von Neumann do operador estatístico reduzido $\hat{\rho}^{(C)}(3.58)$ como uma medida no sistema de três qubits. Usando Eqs. (3.65), (3.28) e (3.29), encontramos

$$
\begin{aligned}
& E(\Psi)=-\operatorname{tr}\left(\hat{\rho}^{(C)} \log _{2} \hat{\rho}^{(C)}\right)=-\sum_{i=1,2} \lambda_{i} \log _{2} \lambda_{i} \\
& =-\left[z \log _{2} z+(1-z) \log _{2}(1-z)\right]
\end{aligned}
$$

onde

$$
z=\frac{1+y}{2}, \quad y=\sqrt{\left(\rho_{11}^{(C)}-\rho_{22}^{(C)}\right)^{2}+4\left|\rho_{12}^{(C)}\right|^{2}} .
$$

Expressando os elementos da matriz densidade $\rho_{a b}^{(C)}$ via coeficientes $v_{i}, i=$ $1, \ldots, 8$, de acordo com Eqs. (3.58) e (3.59), obtemos

$$
\begin{aligned}
y= & {\left[\left(\left|v_{1}\right|^{2}+\left|v_{3}\right|^{2}+\left|v_{5}\right|^{2}+\left|v_{7}\right|^{2}-\left|v_{2}\right|^{2}-\left|v_{4}\right|^{2}-\left|v_{6}\right|^{2}-\left|v_{8}\right|^{2}\right)^{2}\right.} \\
& \left.+4\left|v_{1} v_{2}^{*}+v_{3} v_{4}^{*}+v_{5} v_{6}^{*}+v_{7} v_{8}^{*}\right|^{2}\right]^{1 / 2}
\end{aligned}
$$

onde

$$
\begin{aligned}
& v_{1}=\vartheta_{000}, v_{2}=\vartheta_{001}, v_{3}=\vartheta_{010}, v_{4}=\vartheta_{011}, \\
& v_{5}=\vartheta_{100}, v_{6}=\vartheta_{101}, v_{7}=\vartheta_{110}, v_{8}=\vartheta_{111} \text {. }
\end{aligned}
$$




\section{Capítulo 4}

\section{Emaranhamento em feixes de fótons com frequências diferentes}

\subsection{Emaranhamento em dois feixes de fótons}

Para ilustrar a ideia proposta, consideramos o caso quando temos dois fótons com frequências diferentes no estado inicial. Escolhemos dois fótons com frequências $\kappa_{s_{1}}$ e $\kappa_{s_{2}}$ onde $\kappa_{s_{1}}=\kappa_{0} n$ e $\kappa_{s_{2}}=\kappa_{0} r, r, n \in \mathbb{N}, r>n, \kappa_{0}=2 \pi / L=$ $2 \pi \rho^{1 / 3}$. Também consideramos o caso quando o parâmetro $\epsilon$ é pequeno. Por isso, desprezamos termos da ordem $o(\epsilon)$. Neste caso, podemos usar fórmulas (2.65) para raízes $r_{k \lambda}$ e matrizes $u_{s \lambda, k \lambda^{\prime}}$.

\subsubsection{Fótons com polarizações antiparalelas}

Vamos considerar primeiro o vetor de estado (2.63) com apenas dois quasefótons, um do primeiro tipo, com frequência $\kappa_{r}$, e outro do segundo tipo, com frequência $\kappa_{b}$, e com polarizações antiparalelas, que tomamos como $\lambda_{1}=2 \mathrm{e}$ $\lambda_{2}=1$. Nesses vetores de estado $N_{s_{1}, 1}=N_{s_{2}, 2}=0$ e $N_{s_{1}, 2}=N_{s_{2}, 1}=1$, tal que

$$
\Psi_{\gamma}(\downarrow, \uparrow)=c_{s_{1}, 2}^{+} c_{s_{2}, 1}^{+}|0\rangle_{c} .
$$

Do ponto de vista dos quase-fótons, este é um estado separável. No entanto, se um observador analisa esse estado por meio de ferramentas que registram fótons livres (para ser consistente, temos de supor que, na região onde 
tais medidas são realizadas, o campo magnético e a densidade de elétrons são zero), ele/ela observaram um estado emaranhado de dois fótons livres. O vetor de estado correspondente pode ser obtido a partir da eq. (4.1), expressando todos os operadores de quase-fóton e vetores de vácuo, em termos das correspondentes quantidades de fótons livres. Nesta análise, nós desprezamos todos os termos da ordem $o(\epsilon)$. Nós podemos verificar facilmente que, nesta aproximação, é meramente possível substituir o vácuo de quase-fóton $|0\rangle_{c}$ pelo correspondente vácuo de fóton livre $|0\rangle, a_{s, \lambda}|0\rangle=0$, tal que

$$
\Psi_{\gamma}(\downarrow, \uparrow)=c_{s_{1}, 2}^{+} c_{s_{2}, 1}^{+}|0\rangle_{c} \simeq c_{s_{1}, 2}^{+} c_{s_{2}, 1}^{+}|0\rangle
$$

Os operadores $c_{s_{1}, 2}^{+}$e $c_{s_{2}, 1}^{+}$têm de ser expressos em termos dos operadores de fótons livres usando transformações canônicas (2.58),

$$
c_{s_{1}, 2}^{+}=\sum_{s ; \lambda=1,2} a_{s, \lambda}^{+} u_{s \lambda}, \quad c_{s_{2}, 1}^{+}=\sum_{s ; \lambda=1,2} a_{s, \lambda}^{+} \tilde{u}_{s \lambda}, u_{s \lambda}=u_{s \lambda, s_{1} 2}, \tilde{u}_{s \lambda}=u_{s \lambda, s_{2} 1} .
$$

Dessa forma,

$$
\Psi_{\gamma}(\downarrow, \uparrow) \simeq \sum_{s, s^{\prime}, \lambda, \lambda^{\prime}} u_{s \lambda} \tilde{u}_{s^{\prime} \lambda^{\prime}} a_{s, \lambda}^{+} a_{s^{\prime}, \lambda^{\prime}}^{+}|0\rangle .
$$

Em seguida, podemos ver que, na aproximação sob consideração, nós temos de desprezar termos da forma $u_{k \lambda} \tilde{u}_{s \lambda^{\prime}} a_{k, \lambda}^{+} a_{s, \lambda^{\prime}}^{+}$, com $k, s \neq s_{1}, s_{2}$ e $k=s$ no lado direito da eq. (4.3). Assim, obtemos

$$
\Psi_{\gamma}(\downarrow, \uparrow)=\sum_{\lambda, \lambda^{\prime}}\left[u_{s_{1} \lambda} \tilde{u}_{s_{2} \lambda^{\prime}}+u_{s_{2} \lambda^{\prime}} \tilde{u}_{s_{1} \lambda}\right] a_{s_{1}, \lambda}^{+} a_{s_{2}, \lambda^{\prime}}^{+}|0\rangle .
$$

Introduzindo a base computacional,

$$
\begin{aligned}
& \left|(\lambda-1)\left(\lambda^{\prime}-1\right)\right\rangle=a_{s_{1}, \lambda}^{+} a_{s_{2}, \lambda^{\prime}}^{+}|0\rangle,\left|\vartheta_{1}\right\rangle=|00\rangle, \\
& \left|\vartheta_{2}\right\rangle=|01\rangle,\left|\vartheta_{3}\right\rangle=|10\rangle,\left|\vartheta_{4}\right\rangle=|11\rangle,
\end{aligned}
$$


nós reescrevemos o vetor (4.4) como

$$
\begin{aligned}
& \Psi_{\gamma}(\downarrow, \uparrow)=\sum_{\lambda, \lambda^{\prime}}\left[u_{s_{1} \lambda} \tilde{u}_{2 \lambda^{\prime}}+u_{2 \lambda^{\prime}} \tilde{u}_{s_{1} \lambda}\right]\left|(\lambda-1)\left(\lambda^{\prime}-1\right)\right\rangle \\
& =\sum_{j=1}^{4} v_{j}\left|\vartheta_{j}\right\rangle, \quad \sum_{i=1}^{4}\left|v_{i}\right|^{2}=1 ; \\
& v_{1}=u_{s_{1} 1} \tilde{u}_{s_{2} 1}+u_{s_{2} 1} \tilde{u}_{s_{1} 1}=u_{s_{1} 1, s_{1} 2} u_{s_{2} 1, s_{2} 1}+u_{s_{2} 1, s_{1} 2} u_{s_{1} 1, s_{2} 1}, \\
& v_{2}=u_{s_{1} 1} \tilde{u}_{s_{2} 2}+u_{s_{2} 2} \tilde{u}_{s_{1} 1}=u_{s_{1} 1, s_{1} 2} u_{s_{2} 2, s_{2} 1}+u_{s_{2} 2, s_{1} 2} u_{s_{1} 1, s_{2} 1}, \\
& v_{3}=u_{s_{1} 2} \tilde{u}_{s_{2} 1}+u_{s_{2} 1} \tilde{u}_{s_{1} 2}=u_{s_{1} 2, s_{1} 2} u_{s_{2} 1, s_{2} 1}+u_{s_{2} 1, s_{1} 2} u_{s_{1} 2, s_{2} 1}, \\
& v_{4}=u_{s_{1} 2} \tilde{u}_{s_{2} 2}+u_{s_{2} 2} \tilde{u}_{s_{1} 2}=u_{s_{1} 2, s_{1} 2} u_{s_{2} 2, s_{2} 1}+u_{s_{2} 2, s_{1} 2} u_{s_{1} 2, s_{2} 1} .
\end{aligned}
$$

A medida de emaranhamento do sistema em questão pode ser calculada através da entropia da informação $E_{I}(\Psi)$ (a entropia de von Neumann com a constante de Boltzmann é igual a $1 / \ln 2$ ) do operador densidade reduzida $\hat{\rho}^{(1)}$ do subsistema do primeiro fóton, de acordo com fórmulas (3.47) e (3.48) [44]. Os mesmos resultados podem ser obtidos se calcularmos a entropia de von Neumann do operador reduzido $\hat{\rho}^{(2)}$ do subsistema do segundo fóton. Então,

$$
\begin{aligned}
& E_{I}(\Psi)=-\operatorname{tr}\left(\hat{\rho}^{(1)} \log _{2} \hat{\rho}^{(1)}\right)=-\sum_{a=1,2} \lambda_{a} \log _{2} \lambda_{a}, \\
& \hat{\rho}^{(1)}=\operatorname{tr}_{2} \hat{\rho}, \hat{\rho}=\left|\Psi_{\gamma}(\downarrow, \uparrow)\right\rangle\left\langle\Psi_{\gamma}(\downarrow, \uparrow),\right|
\end{aligned}
$$

onde $\lambda_{a}, a=1,2$, são os autovalores de $\hat{\rho}^{(1)}$. No que segue, nós chamaremos $E_{I}(\Psi)$ a medida de informação. Os autovalores $\lambda_{a}$ e a medida de informação $E_{E}(\Psi)$ têm a seguinte forma

$$
\begin{aligned}
& \lambda_{a}=\frac{1}{2}\left[\rho_{11}^{(1)}+\rho_{22}^{(1)}+(-1)^{a} y\right], \quad y=\sqrt[+]{\left(\rho_{11}^{(1)}-\rho_{22}^{(1)}\right)^{2}+4\left|\rho_{12}^{(1)}\right|^{2}}, \\
& E_{I}(\Psi)=-\frac{1}{\ln 4}\left[(1-y) \ln \left(\frac{1-y}{2}\right)+(1+y) \ln \left(\frac{1+y}{2}\right)\right] .
\end{aligned}
$$

Para obter a quantidade $E_{I}(\Psi)$ no nosso caso específico, usamos a forma explícita (2.65) das matrizes $u$ e das raízes $r_{k \lambda}$ para calcular as quantidades $v_{i}$. Sendo esses últimos

$$
v_{1}=-(a+b), \quad v_{2}=-i(a-b), \quad v_{3}=-v_{2}, \quad v_{4}=v_{1},
$$


onde

$$
\begin{aligned}
& a=\left[\sqrt{\kappa_{s_{2}} r_{s_{1}, \lambda}}\left(r_{s_{1}, \lambda}-\kappa_{s_{2}}\right) \sqrt{\kappa_{s_{1}} r_{s_{2}, \lambda \prime}}\left(r_{s_{2}, \lambda}-\kappa_{s_{1}}\right) P\right]^{-1}, \\
& b=\left[\sqrt{\kappa_{s_{1}} r_{s_{1}, \lambda}}\left(r_{s_{1}, \lambda}-\kappa_{s_{1}}\right) \sqrt{\kappa_{s_{2}} r_{s_{2}, \lambda}}\left(r_{s_{2}, \lambda \lambda}-\kappa_{s_{2}}\right) P\right]^{-1}, \\
& P=4\left[\left(\frac{2}{\left(r_{s_{1}, \lambda}^{2}-\kappa_{s_{1}}^{2}\right)^{2}}+\frac{2}{\left(r_{s_{1}, \lambda}^{2}-\kappa_{s_{2}}^{2}\right)^{2}}-\frac{\omega}{\epsilon r_{s_{1}, \lambda}^{3}}\right)\right. \\
& \left.\left(\frac{2}{\left(r_{s_{2}, \lambda^{\prime}}^{2}-\kappa_{s_{1}}^{2}\right)^{2}}+\frac{2}{\left(r_{s_{2}, \lambda^{\prime}}^{2}-\kappa_{s_{2}}^{2}\right)^{2}}+\frac{\omega}{\epsilon r_{s_{2}, \lambda^{\prime}}^{3}}\right)\right]^{1 / 2} .
\end{aligned}
$$

Em seguida, obtemos a quantidade $y$ da eq. (4.7) na aproximação em questão, assim

$$
\begin{aligned}
& y(\downarrow, \uparrow)=4\left|\left(a^{2}-b^{2}\right)\right|=1-\epsilon \Phi(\downarrow, \uparrow), \quad 0 \leq \epsilon \Phi(\downarrow, \uparrow)<1, \\
& \Phi(\downarrow, \uparrow)=\frac{\omega}{2}\left[\frac{1}{\kappa_{s_{2}}\left(\omega-\kappa_{s_{2}}\right)^{2}}-\frac{1}{\kappa_{s_{1}}\left(\kappa_{s_{1}}+\omega\right)^{2}}\right] .
\end{aligned}
$$

Avaliando o comportamento assintótico da medida de informação $E_{I}\left(\Psi_{\gamma}(\uparrow, \downarrow)\right)$ conforme $\epsilon \rightarrow 0$ resulta

$$
E_{I}\left(\Psi_{\gamma}(\downarrow, \uparrow)\right)=\frac{\Phi(\downarrow, \uparrow)}{2 \ln 2}[\epsilon(1-\ln (\Phi(\downarrow, \uparrow) / 2))-\epsilon \ln \epsilon] .
$$

Nós calculamos a medida de emaranhamento para diferentes valores de frequências do cíclotron $\omega=0 \div 0.5 \mathrm{THz}$ atingíveis atualmente no laboratório. Para este estudo, foram selecionadas as frequências angulares do fóton, começando com a luz vermelha $\kappa_{s_{1}}=2500 T H z$ e a $E_{I}\left(\Psi_{\gamma}(\downarrow, \uparrow)\right)$, calculada como uma função de $\Delta \kappa=\kappa_{s_{2}}-\kappa_{s_{1}}$, que varia do vermelho ao ultravioleta. O resultado é mostrado na Figura 4.1, como um gráfico de superfície, onde o gradiente de cor representa os valores da medida de emaranhamento.

Sabemos também que, para um estado qubit bipartido puro, é possível identificar o emaranhamento avaliando a denominada medida de Schmidt $E_{S}(\Psi)$, que é o traço do quadrado dos operadores de densidade reduzida $[57]$

$$
E_{S}(\Psi(\downarrow, \uparrow))=-\operatorname{tr}\left[\left(\hat{\rho}^{(1)}\right)^{2}\right]=-\sum_{a=1,2} \lambda_{a}^{2} .
$$




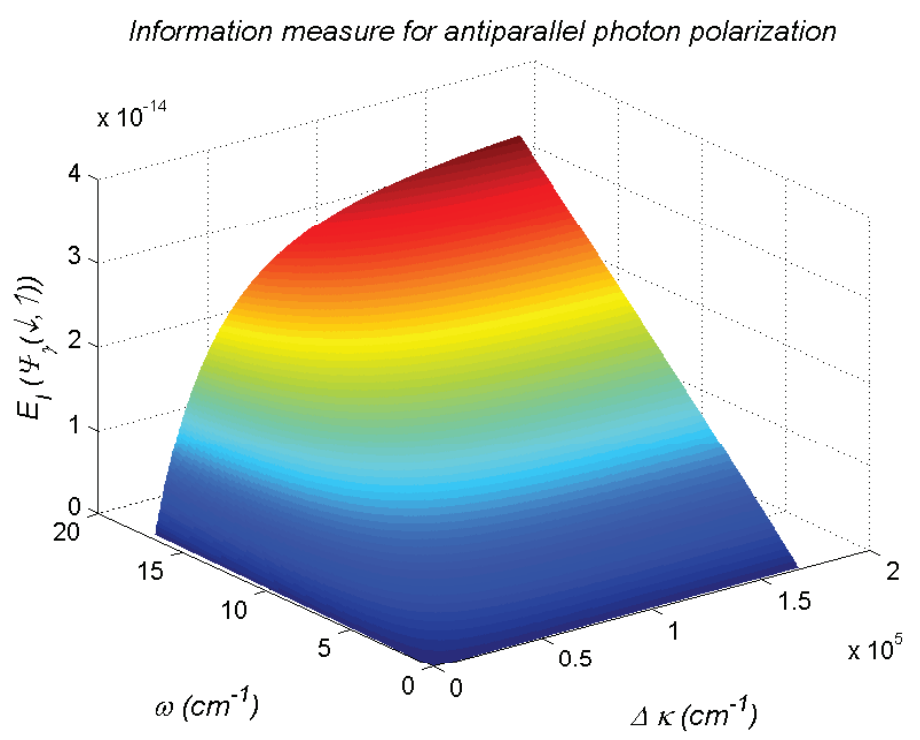

Figura 4.1: Medida de informação $E_{I}\left(\Psi_{\gamma}(\downarrow, \uparrow)\right)$ como uma função de $\omega$ e $\Delta \kappa$, para $\epsilon=0.1$.

A medida de Schmidt pode ser considerada como uma alternativa à medida de emaranhamento da informação. No caso em análise

$$
\begin{aligned}
& E_{S}\left(\Psi_{\gamma}(\downarrow, \uparrow)\right)=1-\left[\left(\rho_{11}^{(1)}\right)^{2}+\left(\rho_{22}^{(1)}\right)^{2}+2\left|\rho_{12}^{(1)}\right|^{2}\right] \\
& =1-\left[2\left(\left|v_{1}\right|^{2}+\left|v_{2}\right|^{2}\right)^{2}+8\left|v_{1} v_{2}\right|^{2}\right]=1-16\left(a^{4}+b^{4}\right)=2 \epsilon \Phi(\downarrow, \uparrow) .
\end{aligned}
$$

A medida de Schmidt calculada para os parâmetros escolhidos anteriormente tem um comportamento bastante semelhante quanto à medida da informação e é apresentado na Figura 4.2

\subsubsection{Fótons com polarizações paralelas alinhadas ao longo do campo magnético}

Vamos reconsiderar estados (2.63), de novo, com dois quase-fótons, um do primeiro tipo e outro de segundo tipo, mas com polarizações paralelas $\lambda_{1}=1$ e $\lambda_{2}=1$. Esse vetor de estado corresponde a $N_{s_{1}, 1}=N_{s_{2}, 1}=1$ e $N_{s_{1}, 2}=$ 


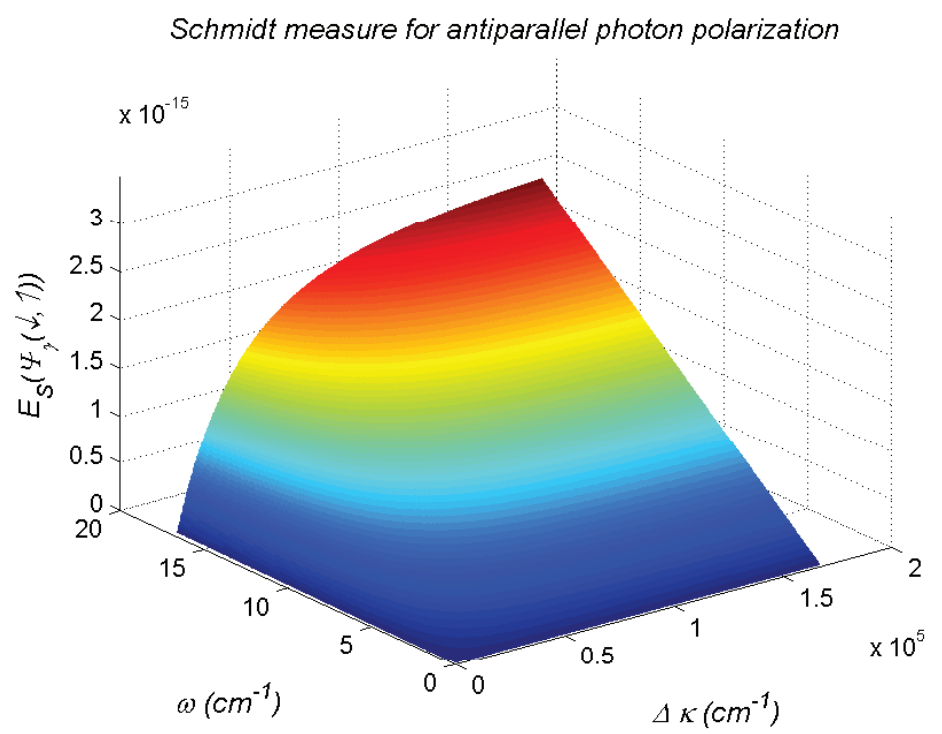

Figura 4.2: Medida de Schmidt $E_{S}\left(\Psi_{\gamma}(\downarrow, \uparrow)\right)$ como uma função de $\omega$ e $\Delta \kappa$, para $\epsilon=0.1$.

$N_{s_{2}, 2}=0$ e tem a forma

$$
\Psi_{\gamma}(\uparrow, \uparrow)=c_{s_{1}, 1}^{+} c_{s_{2}, 1}^{+}|0\rangle_{c}
$$

Empregando os mesmos argumentos que foram utilizados no caso de polarizações antiparalelas, obtemos para $\Psi_{\gamma}(\uparrow, \uparrow)$ representação (4.5) com

$$
\begin{array}{ll}
v_{1}=u_{s_{1} 1, s_{1} 1} u_{s_{2} 1, s_{2} 1}+u_{s_{2} 1, s_{1} 1} u_{s_{1} 1, s_{2} 1}, & v_{2}=u_{s_{1} 1, s_{1} 1} u_{s_{2} 2, s_{2} 1}+u_{s_{2} 2, s_{1} 1} u_{s_{1} 1, s_{2} 1} \\
v_{3}=u_{s_{1} 2, s_{1} 1} u_{s_{2} 1, s_{2} 1}+u_{s_{2} 1, s_{1} 1} u_{s_{1} 2, s_{2} 1}, & v_{4}=u_{s_{1} 2, s_{1} 1} u_{s_{2} 2, s_{2} 1}+u_{s_{2} 2, s_{1} 1} u_{s_{1} 2, s_{2} 1}
\end{array}
$$

Utilizando a forma explícita das matrizes $u$ da eq. (2.64) e as raízes quadradas $r_{k \lambda}$ da eq. (2.65), calculamos as grandezas $v_{i}$. Elas são

$$
v_{1}=a+b, v_{2}=-i v_{1}, v_{3}=-i v_{1}, v_{4}=-v_{1}
$$


onde

$$
\begin{aligned}
a & =\left[\sqrt{\kappa_{s_{2}} r_{s_{1}, \lambda}}\left(r_{s 1, \lambda}-\kappa_{s_{2}}\right) \sqrt{\kappa_{s_{1}} r_{s_{2}, \lambda}}\left(r_{s_{2}, \lambda}-\kappa_{s_{1}}\right) P\right]^{-1}, \\
b & =\left[\sqrt{\kappa_{s_{1}} r_{s_{1}, \lambda}}\left(r_{s 1, \lambda}-\kappa_{s_{1}}\right) \sqrt{\kappa_{s_{2}} r_{s_{2}, \lambda}}\left(r_{s_{2}, \lambda}-\kappa_{s_{2}}\right) P\right]^{-1}, \\
P & =4\left[\left(\frac{2}{\left(r_{s_{1}, \lambda}^{2}-\kappa_{s_{1}}^{2}\right)^{2}}+\frac{2}{\left(r_{s_{1}, \lambda}^{2}-\kappa_{s_{2}}^{2}\right)^{2}}-\frac{\omega}{\epsilon r_{s_{1}, \lambda}^{3}}\right)\right. \\
& \left.\left(\frac{2}{\left(r_{s_{2}, \lambda}^{2}-\kappa_{s_{1}}^{2}\right)^{2}}+\frac{2}{\left(r_{s_{2}, \lambda}^{2}-\kappa_{s_{2}}^{2}\right)^{2}}-\frac{\omega}{\epsilon r_{s_{2}, \lambda}^{3}}\right)\right]^{1 / 2} .
\end{aligned}
$$

Em seguida, obtemos a grandeza $y$

$$
y=\sqrt{4\left|v_{1} i v_{1}+\left(-i v_{1}\right)\left(-v_{1}\right)\right|^{2}}=4\left|v_{1}^{2}\right|=4\left|(a+b)^{2}\right|=1 .
$$

Podemos ver facilmente que, neste caso, tanto a informação quanto a medida de emaranhamento de Schmidt do estado $\Psi_{\gamma}(\uparrow, \uparrow)$ são zero

$$
E_{I}\left(\Psi_{\gamma}(\uparrow, \uparrow)\right)=0, E_{S}\left(\Psi_{\gamma}(\uparrow, \uparrow)\right)=0 .
$$

O mesmo resultado se aplica para o estado

$$
\Psi_{\gamma}(\downarrow, \downarrow)=c_{s_{1}, 2}^{+} c_{s_{2}, 2}^{+}|0\rangle_{c}
$$

com dois quase-fótons, um do primeiro tipo, e um outro do segundo tipo e com polarizações paralelas direcionados contra o campo magnético, $\lambda_{1}=$ $2, \lambda_{2}=2$

$$
E_{I}\left(\Psi_{\gamma}(\downarrow, \downarrow)\right)=E_{S}\left(\Psi_{\gamma}(\downarrow, \downarrow)\right)=0 .
$$

\subsection{Emaranhamento em três feixes de fótons}

Considere, agora, o vetor de estado (2.63) com três quase-fótons com diferentes frequências. Supomos que o quase-fóton de primeiro tipo e o quasefóton de segundo tipo têm polarizações antiparalelas, que tomaremos como $\lambda_{1}=2$ e $\lambda_{2}=1$, ao passo que a polarização do quase-fóton de terceiro tipo é paralelo à polarização do quase-fóton do segundo tipo, tal que $\lambda_{3}=1$. Esse vetor de estado satisfaz $N_{1,1}=N_{2,2}=N_{3,2}=0$ e $N_{1,2}=N_{2,1}=N_{3,1}=1 \mathrm{e}$ tem a forma

$$
\Psi_{\gamma}(\downarrow, \uparrow, \uparrow)=c_{1,2}^{+} c_{2,1}^{+} c_{3,1}^{+}|0\rangle_{c}, \quad|0\rangle_{c}=\left|0_{1}\right\rangle_{c} \otimes\left|0_{2}\right\rangle_{c} \otimes\left|0_{3}\right\rangle_{c} .
$$


Do ponto de vista dos quase-fótons, este é um estado separável. No entanto, se um observador analisa esse estado por meio de ferramentas que registram fótons livres (para ser consistente, temos de supor que, na região onde tais medidas são realizadas, o campo magnético e a densidade de elétrons são zero), ele/ela observaram um estado emaranhado de três fótons livres. $\mathrm{O}$ vetor de estado correspondente pode ser obtido a partir da eq. (4.21), expressando todos os operadores de quase-fóton e vetores de vácuo, em termos das correspondentes quantidades de fótons livres. Nesta análise, nós desprezamos todos os termos da ordem $o(\epsilon)$. Podemos, facilmente, verificar que, nesta aproximação, é meramente possível substituir o vácuo de quase-fóton $\left|0_{s}\right\rangle_{c}$ pelo correspondente vácuo de fóton livre $|0\rangle_{s}, a_{s, \lambda}\left|0_{s}\right\rangle=0$, $s=1,2,3$, de modo que

$$
\Psi_{\gamma}(\downarrow, \uparrow, \uparrow)=c_{1,2}^{+} c_{2,1}^{+} c_{3,1}^{+}|0\rangle_{c} \simeq c_{1,2}^{+} c_{2,1}^{+} c_{3,1}^{+}|0\rangle, \quad|0\rangle=\left|0_{1}\right\rangle \otimes\left|0_{2}\right\rangle \otimes\left|0_{3}\right\rangle
$$

Os operadores $c_{1,2}^{+}, \quad c_{2,1}^{+}$e $c_{3,1}^{+}$têm que ser expressos em termos dos operadores de fótons livres usando transformações canônicas (2.58), derivados para o caso de três fótons,

$$
\begin{aligned}
& c_{1,2}^{+}=\sum_{s=1}^{3} \sum_{\lambda=1,2} a_{s, \lambda}^{+} u_{s \lambda}, \quad u_{s \lambda}=u_{s \lambda, 12}, \\
& c_{2,1}^{+}=\sum_{s=1}^{3} \sum_{\lambda=1,2} a_{s, \lambda}^{+} \tilde{u}_{s \lambda}, \quad \tilde{u}_{s \lambda}=u_{s \lambda, 21}, \\
& c_{3,1}^{+}=\sum_{s=1}^{3} \sum_{\lambda=1,2} a_{s, \lambda}^{+} \breve{u}_{s \lambda}, \quad \breve{u}_{s \lambda}=u_{s \lambda, 31} .
\end{aligned}
$$

Em seguida, podemos ver que, na aproximação em análise, nós temos de desprezar termos da forma $a_{s, \lambda}^{+} a_{s, \lambda^{\prime}}^{+} a_{s^{\prime \prime}, \lambda^{\prime \prime}}^{+}$e assim por diante no lado direito 
da eq. (4.22). Dessa forma, obtemos

$$
\begin{aligned}
& \Psi_{\gamma}(\downarrow, \uparrow, \uparrow) \simeq \sum_{s, s^{\prime}, \lambda, \lambda^{\prime}} u_{s \lambda} \tilde{u}_{s^{\prime} \lambda^{\prime}} \breve{u}_{s^{\prime \prime} \lambda^{\prime \prime}} a_{s, \lambda}^{+} a_{s^{\prime}, \lambda^{\prime}}^{+} a_{s^{\prime \prime}, \lambda^{\prime \prime}}^{+}|0\rangle \\
& =\sum_{\lambda, \lambda^{\prime}}\left[u_{1 \lambda} \tilde{u}_{2 \lambda^{\prime}} \breve{u}_{3 \lambda^{\prime \prime}} a_{1, \lambda}^{+} a_{2, \lambda^{\prime}}^{+} a_{3, \lambda^{\prime \prime}}^{+}+u_{1 \lambda} \tilde{u}_{3 \lambda^{\prime}} \breve{u}_{2 \lambda^{\prime \prime}} a_{1, \lambda}^{+} a_{3, \lambda^{\prime}}^{+} a_{2, \lambda^{\prime \prime}}^{+}+u_{2 \lambda} \tilde{u}_{1 \lambda^{\prime}} \breve{u}_{3 \lambda^{\prime \prime}} a_{2, \lambda}^{+} a_{1, \lambda^{\prime}}^{+} a_{3, \lambda^{\prime \prime}}^{+}\right. \\
& \left.+u_{2 \lambda} \tilde{u}_{3 \lambda^{\prime}} \breve{u}_{1 \lambda^{\prime \prime}} a_{1, \lambda^{\prime \prime}}^{+} a_{2, \lambda}^{+} a_{3, \lambda^{\prime}}^{+}+u_{3 \lambda} \tilde{u}_{2 \lambda^{\prime}} \breve{u}_{1 \lambda^{\prime \prime}} a_{3, \lambda}^{+} a_{2, \lambda^{\prime}}^{+} a_{1, \lambda^{\prime \prime}}^{+}+u_{3 \lambda} \tilde{u}_{1 \lambda^{\prime}} \breve{u}_{2 \lambda^{\prime \prime}} a_{3, \lambda}^{+} a_{1, \lambda^{\prime}}^{+} a_{2, \lambda^{\prime \prime}}^{+}\right]|0\rangle \\
& =\sum_{\lambda, \lambda^{\prime}}\left[u_{1 \lambda} \tilde{u}_{2 \lambda^{\prime}} \breve{u}_{3 \lambda^{\prime \prime}}+u_{1 \lambda} \tilde{u}_{3 \lambda^{\prime \prime}} \breve{u}_{2 \lambda^{\prime}}+u_{2 \lambda^{\prime}} \tilde{u}_{1 \lambda} \breve{u}_{3 \lambda^{\prime \prime}}+u_{2 \lambda^{\prime}} \tilde{u}_{3 \lambda^{\prime \prime}} \breve{u}_{1 \lambda}\right. \\
& \left.+u_{3 \lambda^{\prime \prime}} \tilde{u}_{2 \lambda^{\prime}} \breve{u}_{1 \lambda}+u_{3 \lambda^{\prime \prime}} \tilde{u}_{1 \lambda} \breve{u}_{2 \lambda^{\prime}}\right] a_{1, \lambda}^{+} a_{2, \lambda^{\prime}}^{+} a_{3, \lambda^{\prime \prime}}^{+}|0\rangle .
\end{aligned}
$$

Introduzindo a base computacional (3.54),

$$
\begin{aligned}
& \left|(\lambda-1)\left(\lambda^{\prime}-1\right)\left(\lambda^{\prime \prime}-1\right)\right\rangle=a_{1, \lambda}^{+} a_{2, \lambda^{\prime}}^{+} a_{3, \lambda^{\prime \prime}}^{+}|0\rangle, \\
& \left|\Theta_{1}\right\rangle=|000\rangle \Longrightarrow \lambda=\lambda^{\prime}=\lambda^{\prime \prime}=1, \quad\left|\Theta_{2}\right\rangle=|001\rangle \Longrightarrow \lambda=\lambda^{\prime}=1, \lambda^{\prime \prime}=2, \\
& \left|\Theta_{3}\right\rangle=|010\rangle \Longrightarrow \lambda=\lambda^{\prime \prime}=1, \lambda^{\prime}=2, \quad\left|\Theta_{4}\right\rangle=|011\rangle \Longrightarrow \lambda=1, \lambda^{\prime}=\lambda^{\prime \prime}=2, \\
& \left|\Theta_{5}\right\rangle=|100\rangle \Longrightarrow \lambda=2, \lambda^{\prime}=\lambda^{\prime \prime}=1, \quad\left|\Theta_{6}\right\rangle=|101\rangle \Longrightarrow \lambda=2, \lambda^{\prime}=1, \lambda^{\prime \prime}=2, \\
& \left|\Theta_{7}\right\rangle=|110\rangle \Longrightarrow \lambda=\lambda^{\prime}=2, \quad \lambda^{\prime \prime}=1, \quad\left|\Theta_{8}\right\rangle=|1111\rangle \Longrightarrow \lambda=\lambda^{\prime}=\lambda^{\prime \prime}=2 .
\end{aligned}
$$

nós reescrevemos o vetor (4.23) como

$$
\Psi_{\gamma}(\downarrow, \uparrow, \uparrow)=\sum_{s=1}^{8} v_{s}\left|\Theta_{s}\right\rangle,
$$

onde

$$
\begin{aligned}
& v_{1}=f(1,1,1), v_{2}=f(1,1,2), v_{3}=f(1,2,1), \quad v_{4}=f(1,2,2), \\
& v_{5}=f(2,1,1), v_{6}=f(2,1,2), v_{7}=f(2,2,1), \quad v_{8}=f(2,2,2), \\
& f\left(\lambda, \lambda^{\prime}, \lambda^{\prime \prime}\right)=u_{1 \lambda} \tilde{u}_{2 \lambda^{\prime}} \breve{u}_{3 \lambda^{\prime \prime}}+u_{1 \lambda} \tilde{u}_{3 \lambda^{\prime \prime}} \breve{u}_{2 \lambda^{\prime}}+u_{2 \lambda^{\prime}} \tilde{u}_{1 \lambda} \breve{u}_{3 \lambda^{\prime \prime}} \\
& +u_{2 \lambda^{\prime}} \tilde{u}_{3 \lambda^{\prime \prime}} \breve{u}_{1 \lambda}+u_{3 \lambda^{\prime \prime}} \tilde{u}_{2 \lambda^{\prime}} \breve{u}_{1 \lambda}+u_{3 \lambda^{\prime \prime}} \tilde{u}_{1 \lambda} \breve{u}_{2 \lambda^{\prime}} .
\end{aligned}
$$

Um operador estatístico que corresponde ao estado puro (4.25) pode ser escrito como

$$
\hat{\rho}_{\gamma}(\downarrow, \uparrow, \uparrow)=\left(\sum_{s=1}^{8} v_{s}\left|\Theta_{s}\right\rangle\right)\left(\sum_{r=1}^{8} v_{r}^{*}\left\langle\Theta_{r}\right|\right) .
$$


Vamos calcular emaranhamento residual $\tau_{A B C}$ como, veja Eq. (3.74),

$$
\begin{aligned}
\tau_{A B C} & =2\left|\sum \vartheta_{i j k} \vartheta_{i^{\prime} j^{\prime} m} \vartheta_{n p k^{\prime}} \vartheta_{n^{\prime} p^{\prime} m^{\prime}} \epsilon_{i i^{\prime}} \epsilon_{j j^{\prime}} \epsilon_{k k^{\prime}} \epsilon_{m m^{\prime}} \epsilon_{n n^{\prime}} \epsilon_{p p^{\prime}}\right| \\
& =4\left|d_{1}-2 d_{2}+4 d_{3}\right|,
\end{aligned}
$$

onde coeficientes $d_{\mu}$ são dados por Eq.(3.75) ou

$$
\begin{aligned}
& d_{1}=v_{1}^{2} v_{8}^{2}+v_{2}^{2} v_{7}^{2}+v_{3}^{2} v_{6}^{2}+v_{4}^{2} v_{5}^{2} \\
& d_{2}=v_{1} v_{4} v_{5} v_{8}+v_{1} v_{3} v_{6} v_{8}+v_{1} v_{2} v_{7} v_{8}+v_{3} v_{4} v_{5} v_{6} \\
& +v_{2} v_{4} v_{5} v_{7}+v_{2} v_{3} v_{6} v_{7} ; d_{3}=v_{1} v_{4} v_{6} v_{7}+v_{2} v_{3} v_{5} v_{8} .
\end{aligned}
$$

Também temos relações

$$
\begin{aligned}
& v_{1}=f(1,1,1)=\vartheta_{000}, v_{2}=f(1,1,2)=\vartheta_{001}, v_{3}=f(1,2,1)=\vartheta_{010}, \\
& v_{4}=f(1,2,2)=\vartheta_{011}, v_{5}=f(2,1,1)=\vartheta_{100}, v_{6}=f(2,1,2)=\vartheta_{101}, \\
& v_{7}=f(2,2,1)=\vartheta_{110}, \quad v_{8}=f(2,2,2)=\vartheta_{111}, \\
& f\left(\lambda, \lambda^{\prime}, \lambda^{\prime \prime}\right)=u_{1 \lambda} \tilde{u}_{2 \lambda^{\prime}} \breve{u}_{3 \lambda^{\prime \prime}}+u_{1 \lambda} \tilde{u}_{3 \lambda^{\prime \prime}} \breve{u}_{2 \lambda^{\prime}}+u_{2 \lambda^{\prime}} \tilde{u}_{1 \lambda} \breve{u}_{3 \lambda^{\prime \prime}} \\
& +u_{2 \lambda^{\prime}} \tilde{u}_{3 \lambda^{\prime \prime}} \breve{u}_{1 \lambda}+u_{3 \lambda^{\prime \prime}} \tilde{u}_{2 \lambda^{\prime}} \breve{u}_{1 \lambda}+u_{3 \lambda^{\prime \prime}} \tilde{u}_{1 \lambda} \breve{u}_{2 \lambda^{\prime}} .
\end{aligned}
$$

Usando expansão de raízes (2.65) em primeira ordem de $\epsilon$ como

$$
r_{1,2}=\kappa_{1}+\epsilon \kappa_{1,2}, \quad r_{2,1}=\kappa_{2}+\epsilon \kappa_{2,1}, \quad r_{3,1}=\kappa_{3}+\epsilon \kappa_{3,1},
$$

achamos que coeficientes $v_{i}$ têm forma

$$
\begin{aligned}
& v_{1}=-a-b, v_{2}=i a+i b, v_{3}=i a+i b, v_{4}=a+b, \\
& v_{5}=-i a-i b, v_{6}=-a-b, v_{7}=-a-b, v_{8}=i a+i b, \\
& a=\frac{1}{2 \sqrt{2}}, \quad b=\frac{\omega \epsilon\left(\kappa_{1} \kappa_{2} \kappa_{3,1}^{2}-\kappa_{2} \kappa_{3} \kappa_{1,2}^{2}+\kappa_{1} \kappa_{3} \kappa_{2,1}^{2}\right)}{2 \sqrt{2}} .
\end{aligned}
$$

Usando fórmulas (3.75) (3.80) e relações (4.31) entre coeficientes $v_{i}$, obtemos em primeira ordem de $\epsilon$ que emaranhamento residual $\tau_{A B C}$ é zero,

$$
E_{I}\left(\Psi_{\gamma}(\downarrow, \uparrow, \uparrow)\right)=\tau_{A B C}=0 .
$$

Vamos encontrar as expansões das quantidades $v_{i}$ ate segunda ordem de $\epsilon$. Para este efeito, é suficiente calcular as raízes $r_{i}$ ate primeira ordem de $\epsilon$. 
Assim, obtemos:

$$
\begin{aligned}
& v_{1}=-D+\frac{c_{2}}{2 \sqrt{2}} \epsilon^{2}, D=\frac{\left(1+b \epsilon-c_{1} \epsilon^{2}\right)}{2 \sqrt{2}}, \\
& b=\omega\left(-\kappa_{1,2}^{2} \kappa_{1}^{-1}+\kappa_{2,1}^{2} \kappa_{2}^{-1}+\kappa_{3,1}^{2} \kappa_{3}^{-1}\right), c_{1}=\frac{c_{11}+\kappa_{2}^{2} c_{12}}{\kappa_{1}^{2} \kappa_{2}^{2}}, \\
& c_{11}=16 \kappa_{1}^{2} \kappa_{2}^{4}\left(\left(\kappa_{1}^{2}-\kappa_{2}^{2}\right)^{-2}+\left(\kappa_{2}^{2}-\kappa_{3}^{2}\right)^{-2}\right) \kappa_{2,1}^{2} \\
& +\kappa_{1}^{2} \kappa_{2,1}^{2}\left(-1+16 \omega \kappa_{2,1}-12 \omega^{2} \kappa_{2,1}^{2}\right)-8 \kappa_{1} \kappa_{2} \omega^{2} \kappa_{2,1}^{2} \kappa_{3}^{-1}\left(\kappa_{1} \kappa_{3,1}^{2}-\kappa_{3} \kappa_{1,2}^{2}\right), \\
& c_{12}=\kappa_{1}^{2} \kappa_{3,1}^{2} \kappa_{3}^{-2}\left[-1+16 \kappa_{3}^{4}\left(\left(\kappa_{1}^{2}-\kappa_{3}^{2}\right)^{-2}+\left(\kappa_{2}^{2}-\kappa_{3}^{2}\right)^{-2}\right)+16 \omega \kappa_{3,1}-12 \omega^{2} \kappa_{3,1}^{2}\right] \\
& +\kappa_{1,2}^{2}\left[-1+16 \kappa_{1}^{4}\left(\left(\kappa_{1}^{2}-\kappa_{2}^{2}\right)^{-2}+\left(\kappa_{1}^{2}-\kappa_{3}^{2}\right)^{-2}\right)+8 \omega^{2} \kappa_{1} \kappa_{3,1}^{2} \kappa_{3}^{-1}\right] \\
& -16 \omega \kappa_{1,2}^{3}-12 \omega^{2} \kappa_{1,2}^{4}, \\
& c_{2}=8\left[\kappa_{1,2} \kappa_{2,1}\left(\kappa_{1}-\kappa_{2}\right)^{-2}+\kappa_{1,2} \kappa_{3,1}\left(\kappa_{1}-\kappa_{3}\right)^{-2}+\kappa_{2,1} \kappa_{3,1}\left(\kappa_{2}-\kappa_{3}\right)^{-2}\right], \quad \text { (4.33) }
\end{aligned}
$$

e

$$
\begin{aligned}
& v_{2}=i\left(D-\frac{B_{2}}{2 \sqrt{2}} \epsilon^{2}\right), v_{3}=i\left(D-\frac{B_{3}}{2 \sqrt{2}} \epsilon^{2}\right) \\
& v_{4}=D+\frac{B_{4}}{2 \sqrt{2}} \epsilon^{2}, \quad v_{5}=-i v_{4}, v_{6}=i v_{3}, v_{7}=i v_{2}, v_{8}=-i v_{1}(
\end{aligned}
$$

onde

$$
B_{2}=c_{2}-\frac{16 \kappa_{1,2} \kappa_{3,1}}{\left(\kappa_{1}-\kappa_{3}\right)^{2}}, B_{3}=c_{2}-\frac{16 \kappa_{1,2} \kappa_{2,1}}{\left(\kappa_{1}-\kappa_{2}\right)^{2}}, B_{4}=c_{2}-\frac{16 \kappa_{2,1} \kappa_{3,1}}{\left(\kappa_{2}-\kappa_{3}\right)^{2}} .
$$

Após tomar em conta as expressões acima, obtemos exatamente que o emaranhamento residual $\tau_{A B C}$ é zero de novo,

$$
\begin{aligned}
& \tau_{A B C}=\frac{M}{65536}\left[32+4 b \epsilon-N \epsilon^{2}\right] \epsilon^{6} \equiv 0 \\
& N=\left(4 c_{1}+c_{2}+B_{2}+B_{3}-B_{4}\right), M=c_{2}^{3}-c_{2}^{2}\left(B_{2}+B_{3}-B_{4}\right) \\
& -c_{2}\left[B_{2}^{2}-2 B_{2}\left(B_{3}-B_{4}\right)+\left(B_{3}+B_{4}\right)^{2}\right] \\
& +B_{2}^{3}+B_{2}^{2}\left(B_{4}-B_{3}\right)-B_{2}\left(B_{3}+B_{4}\right)^{2}+\left(B_{3}-B_{4}\right)\left(B_{3}+B_{4}\right)^{2} .
\end{aligned}
$$




\section{Capítulo 5}

\section{Conclusões}

1. Nesta tese, foi proposta uma experiência de uma possível reprodução de um emaranhamento quântico de feixes de fótons com diferentes frequências, movendo-se em uma mesma direção, controlado por meio de um campo magnético externo. Nessa experiência, a interação entre o campo magnético e fótons é realizada por intermédio de elétrons, que interagem tanto com os fótons quanto com o campo magnético externo.

2. Foi desenvolvida uma teoria que descreve processos físicos nessa experiência. Em primeiro lugar, resolvendo uma equação de Dirac modificada, derivamos estados quânticos do sistema de fótons, em movimentos paralelos, interagindo com o gás dos elétrons relativísticos, sujeitos à ação de um campo magnético. Esses estados podem ser apresentados em termos dos quase-elétrons e quase-fótons, já não interagindo entre si. Para achar o emaranhamento em sistema de dois fótons, com frequências diferentes, precisamos analisar um estado de dois quase-fótons, em termos de fótons livres. A mesma análise foi feita para um sistema de três quase-fótons.

3. Derivamos medidas de emaranhamento de informação e de Schmidt para um sistema geral de dois qubits, e a medida residual para um sistema geral de três qubits.

4. Usando a informação obtida da análise dos sistemas de dois e de três quase-fótons, calculamos medidas de emaranhamento nesses casos. Acontece que a medida residual para um sistema de três fótons é zero na aproximação ate de oitavo ordem no parâmetro pequeno que nos usamos na consideração.

5. Criamos um programa para cálculo numérico através do qual construímos gráficos de dependência das medidas de emaranhamentos em feixes de dois fótons. 
6. Em geral, o emaranhamento aumenta com o crescimento do campo magnético. Note-se que não consideramos os casos de ressonância, onde a frequência do cíclotron se aproxima das frequências de fótons. Obviamente, o emaranhamento depende dos parâmetros que especificam o meio do elétron, tais como a densidade e a energia, e o impulso dos elétrons. Não estudamos essa dependência, essas características foram fixadas pela escolha natural de um pequeno parâmetro em nossos cálculos.

7. Os resultados obtidos nos permitem ver como a medida de emaranhamento depende dos parâmetros, que caracterizam o sistema em questão. Por exemplo, se ambas as polarizações dos fótons coincidem, então, nenhum emaranhamento ocorre. O emaranhamento ocorre apenas se as polarizações do fóton forem opostas.

8. Entendemos que o nosso estudo é baseado em soluções exatas do problema modelo - o elétron interagindo com um campo quantizado de dois fótons e um campo magnético uniforme constante. Em primeiro lugar, o campo magnético uniforme e constante é uma idealização que não pode ser realizada experimentalmente. No entanto, essa idealização nos permite soluções exatas, que são frequentemente usadas em QED. Às vezes, é possível verificar que os processos espaço-temporais locais não dependem essencialmente do comportamento assintótico do campo externo. Mais resultados realistas poderiam ser obtidos se os campos magnéticos utilizados desaparecessem no infinito do espaço-tempo.

9. No cálculo da medida de emaranhamento, também temos utilizado a aproximação de primeira ordem no parâmetro $\epsilon$, supondo que é pequeno, $\epsilon \sim 0.1$. De fato, isso impõe restrições à densidade de elétrons $\rho$ e à integral de movimento $\Lambda$ que caracteriza o elétron. No entanto, essa aproximação foi suficiente para o nosso estudo preliminar semi-qualitativo do problema. Todas as aproximações mencionadas anteriormente teriam de ser cuidadosamente avaliadas, a fim de que os números reais para a eventual realização experimental do emaranhamento controlado de fótons por um campo magnético possam ser extraídos.

10. Por fim, ressaltamos que o objetivo principal do nosso estudo foi demonstrar a possibilidade de emaranhar feixes de fótons com a ajuda de um campo magnético externo. Em contraste com as possibilidades bem conhecidas de se fazer isso usando dispositivos de cristais, a presente forma permite mudar facilmente e de maneira contínua a medida de emaranhamento. Não discutimos, nesta tese, como se pode fazer experimentalmente a medida de emaranhamento. Esse é um importante problema geral, que, no entanto, 
está fora do escopo do nosso estudo. Cada caso específico exige esforços significativos para realizar os experimentos. Resultados importantes, nesse sentido, podem ser encontrados em referências. [20, 21, 22, 23]. A descrição de alguns experimentos que usam métodos de NMR e que permitem detectar emaranhamento quântico pode ser encontrada no livro [17]. 


\section{Referências Bibliográficas}

[1] A. Einstein, B. Podolsky, and N. Rosen, Can quantum mechanical description of physical reality be considered complete, Phys. Rev. 47 (1935) $777-780$

[2] D. Bohm, Quantum Theory, (Prentice-Hall, Englewood Cliffs, 1951)

[3] J.S. Bell, Speakable and unspeakable in quantum mechanics, (Cambridge Univ. Press, N.Y. 1987)

[4] S.J. Freedman and J.F. Clauser, Experimental test of local hidden-varible theories, Phys. Rev. Lett. 28, 938 (1972); E.S. Fry, R.C. Thompson, Experimental test of local hidden-varible theories, Phys. Rev. Lett. 37, 465 (1976); J. G. Weihs, T. Jennewein, C. Simon, H. Weinfurter, A. Zeilinger (1998), Violation of Bell's inequality under strict Einstein locality conditions, Phys. Rev. Lett. 81: 5039; J. Jian-Wei Pan, D. Bouwmeester, M. Daniell, H. Weinfurter \& A. Zeilinger (2000). Experimental test of quantum nonlocality in three-photon GHZ entanglement. Nature 403 (6769): $515-519$.

[5] J. Preskill, Quantum Computation, Lecture Notes, (1997-99), http://www.theory.caltech.edu/people/preskill/ph219/\#lecture

[6] M.A. Nielsen and I.L. Chuang, Quantum computation and quantum information, (Cambridge University Press, Cambridge, 2000).

[7] R. Alicki and M. Fannes, Quantum Dynamical Systems, (Oxford University Press, New York, 2001)

[8] A.K. Ekert, Quantum cryptography based on Bell's theorem, Phys. Rev. Lett. 67, 661 (1991) 
[9] D. Bohm, Quantum Theory, (Englewood Cliffs, NJ: Prentice-Hall 1951)

[10] P.A.M. Dirac, The principles of quantum mechanics, (Clarendon Press, Oxford 1958)

[11] A.S. Davydov, Quantum Mechanics, Sec. ed. (Pergamon Press, Oxford, New York 1976)

[12] A. Messiah, Quantum Mechanics, (Interscience, New York 1961)

[13] L.D. Landau and E.M. Lifshitz, Quantum Mechanics: Non-Relativistic Theory (Pergamon Press, Oxford 1977)

[14] C. Cohen-Tannoudji, B. Diu, and F. Laloë, Quantum Mechanics, (Wiley, New York 1977)

[15] L. E. Ballentine, Quantum Mechanics. A Modern Development, (World Sci. Publish. Singapore 1998)

[16] J. von Neumann, Mathematische Grundlagen der Quantenmechanik, (Verlag von Julius Springer-Verlag, Berlin 1932)

[17] I.S. Oliveira, et al. NMB Quantum Information Processing, (Elsevier, Amsterdam 2007)

[18] D. Campo, R. Parentani, Inflationary spectra and partially decohered distributions, Phys. Rev. D 72, 045015 (2005)

[19] S-Y Lin, C-H Chou, B.L. Hu, Quantum entanglement and entropy in particle creation, Phys. Rev. D 81, 084018 (2010).

[20] J. Adamek, X. Busch, R. Parentani, Dissipative fields in de Sitter and black hole spacetimes: Quantum entanglement due to pair production and dissipation, Phys. Rev. D 87, 124039 (2013)

[21] X. Busch, R. Parentani, Dynamical Casimir effect in dissipative media: When is the final state nonseparable? Phys. Rev. D 88, 045023 (2013)

[22] D E Bruschi, N Friis, I Fuentes and S Weinfurtner, On the robustness of entanglement in analogue gravity systems, New Journ. Phys. 15 (2013) 113016 
[23] S. Finazzi, and I. Carusotto, Entangled phonons in atomic BoseEinstein condensates, arXiv:1309.3414v1 [cond-mat.quant-gas]

[24] S.P. Gavrilov, D.M. Gitman, and A.A. Shishmarev, Statistical properties of states in QED with unstable vacuum. Phys. Rev. A91 (5), 052106 (2015)

[25] I. Ya. Berson, Motion of an electron in an electromagnetic wave and a magnetic field parallel to it, Izv. AN Latv. SSR. Fiz. No. 5, 3-8 (1969).

[26] D. I. Abakarov, V. P. Oleinik, Electron in the field of a quantized electromagnetic wave and in a homogeneous magnetic field, Theor. Math. Phys. 12 No. 1, 77-87 (1972).

[27] M. V. Fedorov, A. E. Kazakov, An electron in a quantized plane wave and in a constant magnetic field, Zeit. Phys. B 261 No. 3, 191-202 (1973).

[28] V. G. Bagrov, D. M. Gitman and P. M. Lavrov, The electron in the quantum field of a plane wave and the classical field of Redmond configuration, Soviet Physics Journal 17 (6), 787-790 (1974).

[29] V. G. Bagrov and D. M. Gitman, Exact Solutions of Relativistic Wave Equations, Math. its Appl., Sov. ser. 39 (Kluwer, Dordrecht 1990); Dirac Equation and its Solutions (de Gruyter, Boston, 2014)

[30] Estudo de emaranhamento num sistema de partículas carregadas em campo de onda plana quantizada, Master thesis by B. de Lima Souza, under the D. Gitman supervision, IFUSP 24/09/2012, unpublished.

[31] D.M. Gitman, I.V. Tyutin, B.L. Voronov, Self-adjoint Extensions in Quantum Mechanics. General theory and applications to Schrödinger and Dirac equations with singular potentials (Birkhauser, Boston 2012)

[32] M. Hazewinkel, Orthogonalization, Encyclopedia of Mathematics, (Springer, 2001)

[33] Artur Ekert and Peter L. Knight, Entangled quantum systems and the Schmidt decomposition, Am. J. Phys. 63 (1995) 415-423

[34] V.B. Berestetsky, E.M. Lifshits, and Pitayevsky, Quantum Electrodynamics, (Nauka, Moscow, 1989). 
[35] A. I. Akhiezer and V. B. Berestetskii, Quantum Electrodynamics, (Interscience 1965).

[36] S. Schweber, An Introduction to Relativistic Quantum Field Theory, (Harper \& Row, New York 1961)

[37] W. Heitler, The Quantum Theory of Radiation, (Oxford Univ. Press, London, 1936)

[38] N.N. Bogoliubov and D.V. Shirkov, Introduction to the Theory of Quantized Fields, 3-rd ed. (John Wiley \& Sons, New York 1980)

[39] D.M. Gitman and I.V. Tyutin, Canonical quantization of fields with constraints, (Nauka, Moscow 1986); Quantization of Fields with Constraints (Springer-Verlag, Berlin 1990)

[40] V.N. Shapovalov, A.V. Shapovalov, Separation of variables in the KleinGordon equation for a superposition of a quantized and classical field, Soviet Physics Journ. 19 (1976) 23-27

[41] V.B. Berestetsky, E.M. Lifshits, L.P. Pitayevsky, Relativistic Quantum Theory. I., (Nauka, Moscow 1968)

[42] F.A. Berezin, The Method of Second Quantization, (Academic Press, New York 1966)

[43] M. Horodecki, P. Horodecki, and R. Horodecki, Separability of mixed states: necessary and sufficient conditions, Phys. Lett. A 223 (1996) 1.

[44] C. H. Bennett, H. J. Bernstein, S. Popescu, and B. Schumacher, Concentrating partial entanglement by local operations, Phys. Rev. A 53, 2046 (1996).

[45] C. H. Bennett, G. Brassard, C. Crépeau, R. Jozsa, A. Peres, and W.K. Wootters, Teleporting an unknown quantum state via dual classical and Einstein-Podolsky-Rosen channels, Phys. Rev. Lett. 70, 1895 (1993)

[46] C. H. Bennett, D. P. DiVincenzo, J. A. Smolin, and W. K. Wootters, Mixed-state entanglement and quantum error correction, Phys. Rev. A 54, 3824 (1996). 
[47] S. Hill and W. K. Wootters, Entanglement of a Pair of Quantum Bits, Phys. Rev. Lett. 78, 5022 (1997).

[48] W. K. Wootters, Entanglement of Formation of an Arbitrary State of Two Qubits, Phys. Rev. Lett. 80, 2245 (1998).

[49] A. Peres, Separability Criterion for Density Matrices, Phys. Rev. Lett. 77, 1413 (1996); M. Horodecki, P. Horodecki, and R. Horodecki, Separability of mixed states: necessary and sufficient conditions, Phys. Lett A 223, 1 (1996).

[50] K. Życzkowski, P. Horodecki, A. Sanpera, and M. Lewenstein, Volume of the set of separable states, Phys. Rev. A 58, 883 (1998).

[51] G. Vidal and R.F. Werner, Computable measure of entanglement, Phys. Rev. A 65, 032314 (2002).

[52] V. Vedral, M.B. Plenio, K. Jacobs, and P.L. Knight, Statistical inference, distinguishability of quantum states, and quantum entanglement, Phys. Rev. A 56, 4452 (1997).

[53] O. Rudolph, Some properties of the computable cross-norm criterion for separability, Phys. Rev. A, 67, 032312 (2003).

[54] J. Lee, M.S. Kim, Caslav Bruker, Operationally Invariant Measure of the Distance between Quantum States by Complementary Measurements, Phys. Rev. Lett. 91, 087902 (2003).

[55] J. Eisert, and H.J. Briegel, Schmidt measure as a tool for quantifying multiparticle entanglement, Phys. Rev. A 64, 022306 (2001).

[56] V. Coffman,J. Kundu, W. K. Wootters, Distributed entanglement, Phys. Rev. A 61 (2000) 052306

[57] J. Eisert, H.J. Briegel, Schmidt measure as a tool for quantifying multiparticle entanglement, Phys. Rev. A 64, 022306 (2001) 


\section{Apêndice A}

\section{Notações}

As coordenadas do espaço de Minkowski em $3+1$ dimensões são indicadas pela

$$
x=\left(x^{\mu}\right)=\left(x^{0}, x^{i}\right), \mu=0,1,2,3, i=1,2,3 .
$$

Nós escrevemos, às vezes, (definindo $c=1$ )

$$
x^{0}=t, x^{1}=x, x^{2}=y, x^{3}=z, \mathbf{r}=\left(x^{i}\right)=(x, y, z), x=(t, \mathbf{r}) .
$$

Adicionalmente,

$$
d x=d x^{0} d x^{1} d x^{2} d x^{3}=d x^{0} d \mathbf{r}, d \mathbf{r}=d x^{1} d x^{2} d x^{3} .
$$

Vetores em três dimensões são indicados por letras em negrito.

Os índices vetoriais e tensoriais Gregos assumem valores 0,1,2,3 e índices Latinos assumem valores 1,2,3. A convenção do somatório sobre índices e expoentes repetidos é sempre assumida, a não ser que de outra forma explicitamente indicada. A métrica no plano do espaço-tempo é determinada pelos tensores de Minkowski $\eta_{\mu \nu}=\operatorname{diag}(1,-1,-1,-1)$, onde, e.g.

$$
\operatorname{diag}(a, b)=\left(\begin{array}{ll}
a & 0 \\
0 & b
\end{array}\right)
$$

Quatro vetores contravariantes e covariantes são representados na forma

$$
\begin{aligned}
& a^{\mu}=\left(a^{0}, a^{i}\right)=\left(a^{0}, \mathbf{a}\right), \mathbf{a}=\left(a^{i}\right), a^{1}=a_{x}, a^{2}=a_{y}, a^{3}=a_{z}, \\
& a_{\mu}=\eta_{\mu \nu} a^{\nu}, a^{\mu}=\eta^{\mu \nu} a_{\nu} .
\end{aligned}
$$


Por meio de $\mathbf{i}, \mathbf{j}$, e $\mathbf{k}$, denotamos três vetores unitários ortogonais entre si, ao longo dos eixos $x, y$, e $z$, respectivamente. Algumas vezes, $\mathbf{i}=\mathbf{e}_{1}, \mathbf{j}=$ $\mathbf{e}_{2}, \mathbf{k}=\mathbf{e}_{3}$. Em seguida

$$
\mathbf{r}=x \mathbf{i}+y \mathbf{j}+z \mathbf{k}=x^{i} \mathbf{e}_{i}, \quad \mathbf{a}=a_{x} \mathbf{i}+a_{y} \mathbf{j}+a_{z} \mathbf{k}=a^{i} \mathbf{e}_{i} .
$$

As notações do tipo $\gamma p=\gamma^{\mu} p_{\mu}$ são usadas em tudo.

Produtos escalares de três e quatro vetores são

$$
\begin{aligned}
& (\mathbf{a b})=a^{i} b^{i}=-a_{i} b^{i}=-a^{i} b_{i}, \mathbf{a}^{2}=(\mathbf{a a})=\left(a^{i}\right)^{2}, \\
& a b=a^{\mu} b_{\mu}=\eta_{\mu \nu} a^{\mu} b^{\nu}=a^{0} b^{0}-(\mathbf{a b}), a^{2}=a^{\mu} a_{\mu}=\left(a^{0}\right)^{2}-\mathbf{a}^{2} .
\end{aligned}
$$

$\mathrm{O}$ produto vetorial de três vetores $\mathbf{a}$ e $\mathbf{b}$ é definido como

$$
\mathbf{c}=[\mathbf{a} \times \mathbf{b}] \Longrightarrow c^{i}=\epsilon^{i j k} a^{j} b^{k},
$$

onde $\epsilon^{i j k}=-\epsilon_{i j k}$ é o tensor de Levi-Civita totalmente antissimétrico em 3 dimensões com a normalização $\epsilon^{123}=1$.

A linha de uma matriz $M$ é denotada pelo índice que está situado do lado esquerdo, e a coluna é denotada pelo índice que está situado do lado direito. Essa combinação não depende da natureza dos índices, sejam eles covariantes ou contravariantes. Por exemplo, $\mu$ é uma linha e $\nu$ é uma coluna de uma matriz $M$ em todos os casos a seguir

$$
M_{\nu}^{\mu}, M_{\mu}^{\nu}, M_{\mu \nu}, M^{\mu \nu} .
$$

Nós, geralmente, denotamos por $I$ a matriz unitária $2 \times 2$, e por $\mathbb{I}$ a matriz unitária $4 \times 4$, e $\delta_{i j}, i, j=1,2,3$, é o delta de Kronecker em 3 dimensões, enquanto que $\delta_{\mu}^{\nu}$ é o delta de Kronecker em $3+1$ dimensões.

As matrizes de Pauli $\boldsymbol{\sigma}=\left(\sigma_{x}, \sigma_{y}, \sigma_{z}\right)$ são

$$
\sigma_{x}=\left(\begin{array}{cc}
0 & 1 \\
1 & 0
\end{array}\right), \sigma_{y}=\left(\begin{array}{cc}
0 & -i \\
i & 0
\end{array}\right), \sigma_{z}=\left(\begin{array}{cc}
1 & 0 \\
0 & -1
\end{array}\right)
$$

Se $A$ é uma matriz, então $A^{*}, A^{T}$, e $A^{+}=A^{* T}$, são usados para denotar o complexo conjugado, o transposto, e o adjunto Hermitiano respectivamente.

Uma sobrelinha denota a conjugação complexa exceto quando indicado. Algumas vezes a conjugação complexa é denotada por $*$ também.

Derivadas parciais:

$$
\frac{\partial A}{\partial x^{\mu}}=\partial_{\mu} A=A_{, \mu}, \frac{\partial}{\partial t}=\partial_{t}=\partial_{0}, \partial_{1}=\partial_{x}, \partial_{2}=\partial_{y}, \partial_{3}=\partial_{z}
$$


O operador $\nabla$, o Laplaciano $\triangle$, e d'Alembertiano $\square$ são definidas da seguinte forma

$$
\begin{aligned}
& \nabla=\mathbf{i} \frac{\partial}{\partial x}+\mathbf{j} \frac{\partial}{\partial y}+\mathbf{k} \frac{\partial}{\partial z}=\mathbf{e}_{i} \partial_{i}=\left(\partial_{i}\right)=\left(\partial_{x}, \partial_{y}, \partial_{z}\right) \\
& \triangle=\nabla^{2}=\partial_{i}^{2}, \square=\partial_{\mu} \partial^{\mu}=\partial_{0}^{2}-\triangle
\end{aligned}
$$

Além disso:

$$
\begin{aligned}
& \operatorname{div} \mathbf{a}=(\boldsymbol{\nabla} \mathbf{a})=\partial_{i} a^{i}=\partial_{x} a_{x}+\partial_{y} a_{y}+\partial_{z} a_{z}, \quad \operatorname{rot} \mathbf{a}=[\boldsymbol{\nabla} \times \mathbf{a}], \\
& \operatorname{grad} \varphi=\boldsymbol{\nabla} \varphi=\mathbf{i} \frac{\partial \varphi}{\partial x}+\mathbf{j} \frac{\partial \varphi}{\partial y}+\mathbf{k} \frac{\partial \varphi}{\partial z}=\mathbf{e}_{i} \partial_{i} \varphi
\end{aligned}
$$

Estaremos usando todo o sistema especial de unidades, onde $\hbar=c=1$ e a lei de Coulomb toma a forma $F=e_{1} e_{2} / 4 \pi r^{2}$ (supõe-se que $e$ é uma carga algébrica de uma partícula, para um elétron $e<0$ ). Chamamos isso de sistema de Heaviside, seguindo a convenção.

As seguintes abreviaturas e notações são utilizadas:

- QED : eletrodinâmica quântica

- QM : mecânica quântica

- $I$ : a matriz unitária $2 \times 2$ e II é a matriz unitária $4 \times 4$

- $\mathbb{N}=\{1,2, \ldots\}$ : o conjunto dos números naturais

- $\mathbb{Z}=\{0, \pm 1, \ldots\}$ : o conjunto dos inteiros

- $\mathbb{Z}_{+}=\{0,1,2, \ldots\}$ : o conjunto dos inteiros positivos

- $\mathbb{R}=(-\infty, \infty)$ : o conjunto de todos os números reais, o eixo real

- $\mathbb{R}^{n}$ : o espaço linear real $n$-dimensional, o conjunto de todos os reais $n$-tuplas $\left(x^{1}, \ldots, x^{n}\right)$

- $\mathbb{C}$ : o conjunto de todos os números complexos, o plano complexo 


\section{Apêndice B}

\section{Anexos}

\section{B.1 Programa para cálculo numérico em Mat- lab}

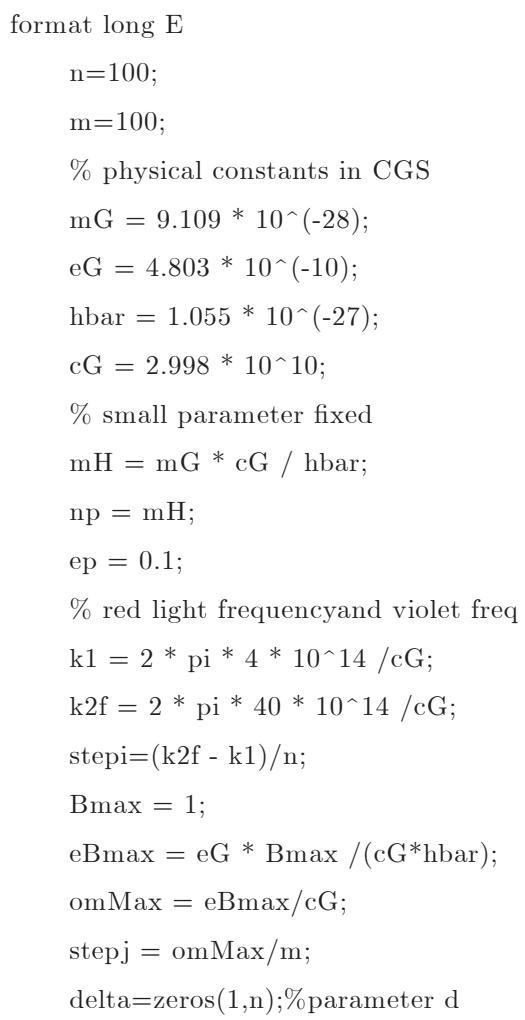


$\mathrm{om}=\operatorname{zeros}(1, \mathrm{~m}) ; \%$ campo

$\% \backslash \mathrm{Phi}$ and $\mathrm{y}$

$\mathrm{P}=\operatorname{zeros}(1, \mathrm{~m})$

$\mathrm{Ent}=\operatorname{zeros}(\mathrm{m}, \mathrm{n}) ; \%$ aprox

$\operatorname{Eschm}=\operatorname{zeros}(\mathrm{m}, \mathrm{n}) ; \%$ aprox

$\mathrm{y}=\operatorname{zeros}(\mathrm{m}, \mathrm{n})$;

$\mathrm{E}=\operatorname{zeros}(\mathrm{m}, \mathrm{n}) ; \%$ formula with logarithms

yant $=\operatorname{zeros}(\mathrm{m}, \mathrm{n})$;

$\operatorname{aant}=\operatorname{zeros}(\mathrm{m}, \mathrm{n})$;

bant $=\operatorname{zeros}(\mathrm{m}, \mathrm{n})$;

$\operatorname{podkvad} 1=\operatorname{zeros}(\mathrm{m}, \mathrm{n}) ; \operatorname{pod} \mathrm{kvad} 2=\operatorname{zeros}(\mathrm{m}, \mathrm{n})$;

$\mathrm{Pag}=\operatorname{zeros}(1, \mathrm{~m})$;

Entag $=\operatorname{zeros}(\mathrm{m}, \mathrm{n})$

$\mathrm{yag}=\operatorname{zeros}(\mathrm{m}, \mathrm{n})$;

$\operatorname{Eag}=\operatorname{zeros}(\mathrm{m}, \mathrm{n})$;

$\mathrm{z}=\mathrm{zeros}(\mathrm{m}, \mathrm{n})$;

$\mathrm{zag}=\operatorname{zeros}(\mathrm{m}, \mathrm{n})$;

$\mathrm{r} 11=\operatorname{zeros}(\mathrm{m}, \mathrm{n})$;

$\mathrm{r} 22=\operatorname{zeros}(\mathrm{m}, \mathrm{n})$;

for $\mathrm{i}=1: \mathrm{n} \%$ photon wave number sweep

$\operatorname{delta}(\mathrm{i})=\mathrm{i} *$ stepi;

$\mathrm{k} 2=\mathrm{k} 1+\operatorname{delta}(\mathrm{i})$;

for $\mathrm{j}=1: \mathrm{m} \%$ magnetic field sweep

$\operatorname{om}(\mathrm{j})=\mathrm{j} *$ stepj;

$\%$ parallel polarization against field

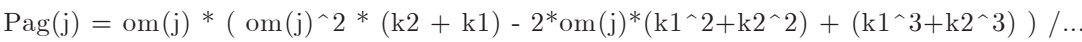

$\left(2^{*} \mathrm{k} 1 * \mathrm{k} 2 *(\mathrm{om}(\mathrm{j})-\mathrm{k} 1) \wedge 2 *(\mathrm{om}(\mathrm{j})-\mathrm{k} 2)^{\wedge} 2\right)$;

$\operatorname{Entag}(\mathrm{j}, \mathrm{i})=\mathrm{ep} *\left(\operatorname{Pag}(\mathrm{j})-\operatorname{Pag}(\mathrm{j}) * \log (\mathrm{ep})-\operatorname{Pag}(\mathrm{j}) * \log (\operatorname{Pag}(\mathrm{j}) / 2) /\left(2^{*} \log (2)\right)\right)$;

$\operatorname{yag}(\mathrm{j}, \mathrm{i})=1-\mathrm{ep} * \operatorname{Pag}(\mathrm{j})$;

$\operatorname{zag}(\mathrm{j}, \mathrm{i})=(1+\operatorname{yag}(\mathrm{j}, \mathrm{i})) / 2$;

$\operatorname{Eag}(\mathrm{j}, \mathrm{i})=-\left(\operatorname{zag}(\mathrm{j}, \mathrm{i}) * \log (\mathrm{zag}(\mathrm{j}, \mathrm{i}))+(1-\mathrm{zag}(\mathrm{j}, \mathrm{i}))^{*} \log (1-\mathrm{zag}(\mathrm{j}, \mathrm{i}))\right) / \log (2)$;

$\%$ antiparallel photon polarization

$\%$ calculate roots of 5 th order equation

$\% \mathrm{r} 11(\mathrm{j}, \mathrm{i})=\mathrm{k} 1+\left(2^{*} \mathrm{k} 1 \wedge 3-\mathrm{k} 1 *(\mathrm{k} 1 \wedge 2+\mathrm{k} 2 \wedge 2)\right)^{*} \mathrm{ep} /$.

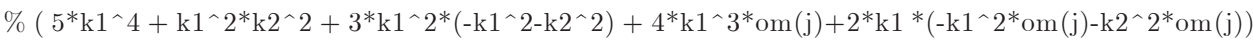

) ;

$\% \mathrm{r} 22(\mathrm{j}, \mathrm{i})=\mathrm{k} 2+\left(2^{*} \mathrm{k} 2 \wedge 3-\mathrm{k} 2 *(\mathrm{k} 1 \wedge 2+\mathrm{k} 2 \wedge 2)\right)^{*} \mathrm{ep} / \ldots$ 


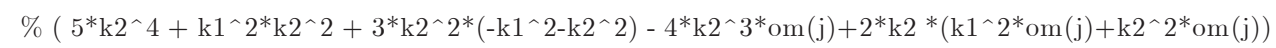
) ;

$\mathrm{r} 11(\mathrm{j}, \mathrm{i})=\mathrm{k} 1+\mathrm{ep} / \ldots$

$\left(2 * \mathrm{k} 1+2^{*} \mathrm{om}(\mathrm{j})\right)$;

$\mathrm{r} 22(\mathrm{j}, \mathrm{i})=\mathrm{k} 2+\mathrm{ep} / \ldots$

$\left(2 * \mathrm{k} 2-2^{*} \mathrm{om}(\mathrm{j})\right)$;

$\%$ calculate $\mathrm{a}$ and $\mathrm{b}$ which compose wave function coefficients

$\operatorname{podkvad} 1(\mathrm{j}, \mathrm{i})=2 /\left(\mathrm{r} 11(\mathrm{j}, \mathrm{i})^{\wedge} 2-\mathrm{k} 1 \wedge 2\right)^{\wedge} 2+2 /(\mathrm{r} 11(\mathrm{j}, \mathrm{i}) \wedge 2-\mathrm{k} 2 \wedge 2)^{\wedge} 2-\mathrm{om}(\mathrm{j}) /(\mathrm{ep} * \mathrm{r} 11(\mathrm{j}, \mathrm{i}) \wedge 3) ;$

$\operatorname{podkvad} 2(\mathrm{j}, \mathrm{i})=2 /\left(\mathrm{r} 22(\mathrm{j}, \mathrm{i})^{\wedge} 2-\mathrm{k} 1 \wedge 2\right)^{\wedge} 2+2 /\left(\mathrm{r} 22(\mathrm{j}, \mathrm{i})^{\wedge} 2-\mathrm{k} 2 \wedge 2\right)^{\wedge} 2+\mathrm{om}(\mathrm{j}) /($ ep * $\mathrm{r} 22(\mathrm{j}, \mathrm{i}) \wedge 3)$;

$\operatorname{aant}(\mathrm{j}, \mathrm{i})=(\operatorname{sqrt}(\mathrm{r} 11(\mathrm{j}, \mathrm{i}) / \mathrm{k} 2)+\operatorname{sqrt}(\mathrm{k} 2 / \mathrm{r} 11(\mathrm{j}, \mathrm{i}))) *(\operatorname{sqrt}(\mathrm{r} 22(\mathrm{j}, \mathrm{i}) / \mathrm{k} 1)+\operatorname{sqrt}(\mathrm{k} 1 / \mathrm{r} 22(\mathrm{j}, \mathrm{i}))) / \ldots$

$\left(4^{*}\left(\mathrm{r} 11(\mathrm{j}, \mathrm{i})^{\wedge} 2-\mathrm{k} 22^{\wedge} 2\right)^{*}\left(\mathrm{r} 22(\mathrm{j}, \mathrm{i})^{\wedge} 2-\mathrm{k} 1 \wedge 2\right) * \ldots\right.$

$\operatorname{sqrt}(\operatorname{podkvad} 1(\mathrm{j}, \mathrm{i})) * \ldots$

$\operatorname{sqrt}(\operatorname{podkvad} 2(\mathrm{j}, \mathrm{i})))$;

$\operatorname{bant}(\mathrm{j}, \mathrm{i})=(\operatorname{sqrt}(\mathrm{r} 11(\mathrm{j}, \mathrm{i}) / \mathrm{k} 1)+\operatorname{sqrt}(\mathrm{k} 1 / \mathrm{r} 11(\mathrm{j}, \mathrm{i}))) *(\operatorname{sqrt}(\mathrm{r} 22(\mathrm{j}, \mathrm{i}) / \mathrm{k} 2)+\operatorname{sqrt}(\mathrm{k} 2 / \mathrm{r} 22(\mathrm{j}, \mathrm{i}))) / \ldots$

$(4 *(\mathrm{r} 11(\mathrm{j}, \mathrm{i}) \wedge 2-\mathrm{k} 1 \wedge 2) *(\mathrm{r} 22(\mathrm{j}, \mathrm{i}) \wedge 2-\mathrm{k} 2 \wedge 2) * \ldots$

$\operatorname{sqrt}(\operatorname{podkvad} 1(\mathrm{j}, \mathrm{i})) * \ldots$

$\operatorname{sqrt}(\operatorname{podkvad} 2(\mathrm{j}, \mathrm{i})))$;

\%calculate y for comparission

$\operatorname{yant}(\mathrm{j}, \mathrm{i})=4 * \operatorname{abs}\left(\operatorname{aant}(\mathrm{j}, \mathrm{i})^{\wedge} 2-\operatorname{bant}(\mathrm{j}, \mathrm{i})^{\wedge} 2\right)$;

$\%$ calculate $\mathrm{y}$ in first approximation

$\mathrm{P}(\mathrm{j})=\mathrm{om}(\mathrm{j}) / 2^{*}\left(-1 /\left((\mathrm{om}(\mathrm{j})+\mathrm{k} 2)^{\wedge} 2^{*} \mathrm{k} 2\right)+1 /\left((\mathrm{om}(\mathrm{j})-\mathrm{k} 1)^{\wedge} 2^{*} \mathrm{k} 1\right)\right) ;$

$\operatorname{Ent}(\mathrm{j}, \mathrm{i})=$ ep $*(\mathrm{P}(\mathrm{j})-\mathrm{P}(\mathrm{j}) * \log (\mathrm{ep})-\mathrm{P}(\mathrm{j}) * \log (\mathrm{P}(\mathrm{j}) / 2) /(2 * \log (2)))$;

$\operatorname{Eschm}(\mathrm{j}, \mathrm{i})=\mathrm{ep}^{*} 2^{*} \mathrm{P}(\mathrm{j})$;

$\mathrm{y}(\mathrm{j}, \mathrm{i})=1-\mathrm{ep} * \mathrm{P}(\mathrm{j})$;

$\mathrm{z}(\mathrm{j}, \mathrm{i})=(1+\mathrm{y}(\mathrm{j}, \mathrm{i})) / 2$;

$\mathrm{E}(\mathrm{j}, \mathrm{i})=-\left(\mathrm{z}(\mathrm{j}, \mathrm{i})^{*} \log (\mathrm{z}(\mathrm{j}, \mathrm{i}))+(1-\mathrm{z}(\mathrm{j}, \mathrm{i}))^{*} \log (1-\mathrm{z}(\mathrm{j}, \mathrm{i}))\right) / \log (2) ;$

end

end

save('AgainstEntropia', 'Entag');

save('Entropia', 'Ent');

$\%$ yant

$\% \mathrm{y}$

$\%$ figure(21)

$\% \operatorname{surfc}($ delta, om, Entag)

$\%$ shading flat;

$\%$ xlabel('\it\{\Delta $\backslash$ kappa $\left.\left(\mathrm{cm}^{\wedge}\{-1\}\right)\right\}^{\prime},{ }^{\prime}$ 'FontSize',12)

$\%$ ylabel('\it $\left\{\backslash\right.$ omega $\left.\left(\mathrm{cm}^{\wedge}\{-1\}\right)\right\}$ ','FontSize',12)

$\%$ zlabel('E (\Psi_\gamma (\downarrow, \downarrow))','FontSize',12) 
$\%$ title('\it\{Entanglement measure for parallel photon polarizations, against field \}','FontSize',12)

$\%$ figure(22)

$\% \operatorname{surfc}($ delta, om, Eag)

$\%$ shading flat;

$\%$ xlabel('\it $\left\{\backslash \text { Delta \kappa }\left(\mathrm{cm}^{\wedge}\{-1\}\right)\right\}^{\prime},{ }^{\prime}$ FontSize',12)

$\%$ ylabel('\it $\left\{\backslash\right.$ omega $\left.\left(\mathrm{cm}^{\wedge}\{-1\}\right)\right\}$ ','FontSize',12)

$\%$ zlabel('E (\Psi_\gamma (\uparrow, \downarrow))','FontSize',12)

$\%$ title('\it\{Entanglement measure for parallel photon polarizations, against field\}','FontSize',12)

$\%$ figure(11)

$\% \operatorname{plot}(\mathrm{om}, \mathrm{P}, \mathrm{om}, \mathrm{Pag})$

$\%$ xlabel('\omega','FontSize',16)

$\%$ ylabel('\Phi_\{antiparal\} , \Phi_\{II\}','FontSize',16,'FontAngle', 'italic')

$\%$ title(' $\backslash$ it $\{$ antiparallel e against the field polarizations\}','FontSize',12)

$\%$

$\%$ figure(12)

$\% \operatorname{surfc}($ delta, om, aant)

$\%$ shading flat;

\% xlabel('\it $\left\{\backslash\right.$ Delta \kappa $\left.\left(\mathrm{cm}^{\wedge}\{-1\}\right)\right\}$ ','FontSize',12)

$\%$ ylabel('\it $\left\{\backslash\right.$ omega $\left.\left(\mathrm{cm}^{\wedge}\{-1\}\right)\right\}$ ','FontSize',12)

$\%$ zlabel('y','FontSize',12)

$\%$ title(' $\backslash$ it $\{$ a for antiparallel photon polarizations\}','FontSize',12)

$\%$

$\%$ figure(13)

$\% \operatorname{surfc}($ delta, om, bant)

$\%$ shading flat;

$\%$ xlabel('\it $\left\{\backslash \text { Delta } \backslash \text { kappa }\left(\mathrm{cm}^{\wedge}\{-1\}\right)\right\}^{\prime},{ }^{\prime}$ FontSize',12)

$\%$ ylabel('\it $\left\{\backslash\right.$ omega $\left.\left(\mathrm{cm}^{\wedge}\{-1\}\right)\right\}$ ','FontSize',12)

$\%$ zlabel('y','FontSize',12)

$\%$ title(' $\backslash$ it $\{\mathrm{b}$ for antiparallel photon polarizations\}','FontSize',12)

$\%$

$\%$ figure(14)

$\% \operatorname{surfc}($ delta, om, podkvad1)

$\%$ shading flat;

$\%$ xlabel(' $\backslash$ it $\left\{\backslash\right.$ Delta $\backslash$ kappa $\left.\left(\mathrm{cm}^{\wedge}\{-1\}\right)\right\}$ ','FontSize',12)

$\%$ ylabel(' $\backslash$ it $\left\{\backslash\right.$ omega $\left.\left(\mathrm{cm}^{\wedge}\{-1\}\right)\right\}$ ','FontSize',12)

$\%$ zlabel('podkvad','FontSize',12)

$\%$ title('\it\{podkvad1 for antiparallel photon polarizations\}','FontSize',12)

$\%$ figure(15) 
$\%$ surfc(delta, om, podkvad2)

$\%$ shading flat;

$\%$ xlabel('\it $\left\{\backslash \text { Delta } \backslash \text { kappa }\left(\mathrm{cm}^{\wedge}\{-1\}\right)\right\}^{\prime}, '$ FontSize',12)

$\%$ ylabel('\it $\left\{\backslash\right.$ omega $\left.\left(\mathrm{cm}^{\wedge}\{-1\}\right)\right\}$ ','FontSize',12)

$\%$ zlabel('podkvad','FontSize',12)

$\%$ title('\it\{podkvad2 for antiparallel photon polarizations \}','FontSize',12)

$\%$ figure(16)

$\% \operatorname{surfc}($ delta, om, r11)

$\%$ shading flat:

$\%$ xlabel('\it $\left\{\backslash \text { Delta } \backslash \text { kappa }\left(\mathrm{cm}^{\wedge}\{-1\}\right)\right\}^{\prime}, '$ FontSize',12)

$\%$ ylabel(' $\backslash$ it $\left\{\backslash\right.$ omega $\left.\left(\mathrm{cm}^{\wedge}\{-1\}\right)\right\}$ ','FontSize',12)

$\%$ zlabel('y','FontSize',12)

$\%$ title('\it\{r11 for antiparallel photon polarizations\}','FontSize',12)

$\%$ figure(17)

$\% \operatorname{surfc}($ delta, om, r22)

$\%$ shading flat;

\% xlabel('\it $\left\{\backslash \text { Delta \kappa }\left(\mathrm{cm}^{\wedge}\{-1\}\right)\right\}^{\prime},{ }^{\prime}$ FontSize',12)

\% ylabel('\it $\left\{\backslash\right.$ omega $\left.\left(\mathrm{cm}^{\wedge}\{-1\}\right)\right\}$ ','FontSize',12)

$\%$ zlabel('y','FontSize',12)

$\%$ title(' $\backslash$ it $\{$ r22 for antiparallel photon polarizations\}','FontSize',12)

figure(18)

$\operatorname{surfc}($ delta, om, Ent)

shading flat;

xlabel(' $\backslash$ it $\{\backslash$ Delta $\backslash$ kappa (cm^\{-1\})\}','FontSize',12)

ylabel('\it $\left\{\backslash\right.$ omega $\left.\left(\mathrm{cm}^{\wedge}\{-1\}\right)\right\}$ ','FontSize',12)

zlabel('\it $\left\{\mathrm{E}_{-} \mathrm{I}\right\}\left(\backslash \mathrm{Psi} \_\backslash\right.$ gamma( $\backslash$ downarrow, \uparrow))','FontSize',12)

title('\it\{Information measure for antiparallel photon polarization\}','FontSize',12)

figure(19)

$\operatorname{surfc}($ delta, om, Eschm)

shading flat;

xlabel(' $\backslash$ it $\left\{\backslash\right.$ Delta $\backslash$ kappa $\left.\left(\mathrm{cm}^{\wedge}\{-1\}\right)\right\}$ ','FontSize',12)

ylabel('\it $\left\{\backslash\right.$ omega $\left.\left(\mathrm{cm}^{\wedge}\{-1\}\right)\right\}$ ','FontSize',12)

zlabel(' \it $\{$ E_S $\}(\backslash P$ si_ $\backslash$ gamma( $\backslash$ downarrow, \uparrow))','FontSize',12)

title('\it $\{$ Schmidt measure for antiparallel photon polarization \}','FontSize',12)

$\% \operatorname{axis}\left(\left[\begin{array}{llllll}0 & 2 \mathrm{E} 5 & 0 & 20 & -1 \mathrm{E}-14 & 1 \mathrm{E}-14\end{array}\right]\right)$

$\%$ figure(31)

$\% \operatorname{surfc}($ delta, om, Ent-Entag)

$\%$ xlabel('\it $\left\{\backslash\right.$ Delta $\left.\backslash k a p p a ~\left(\mathrm{~cm}^{\wedge}\{-1\}\right)\right\}$ ','FontSize',12) 


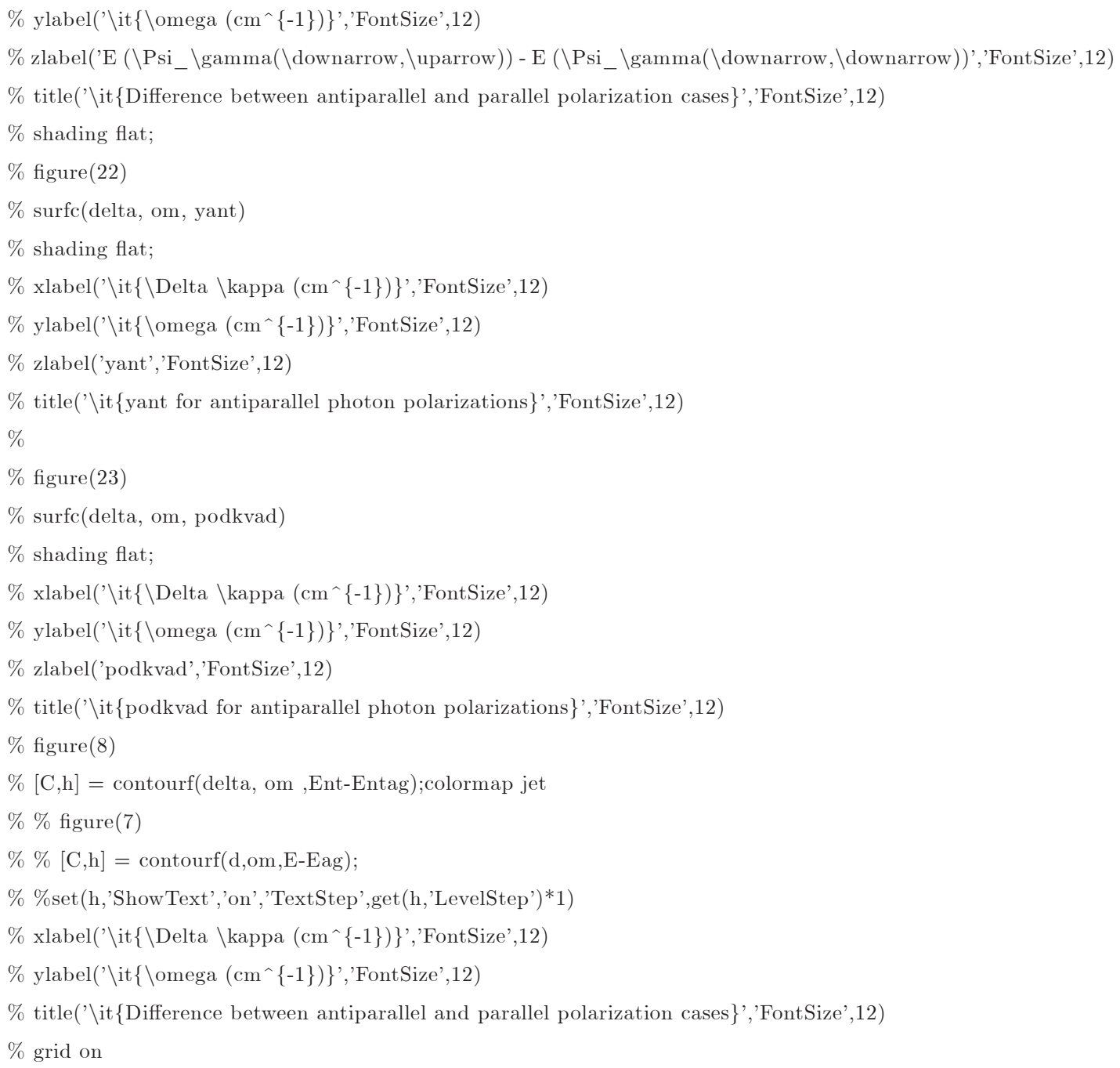




\section{B.2 Caso de dois fótons no Mathematica}

ClearAll ["Global *"]

Clear $[\omega, \epsilon, \lambda, \lambda 1, \lambda 2, \mathbf{k}, \mathbf{s}, \mathrm{q}, \mathrm{u}, \kappa, \vartheta 11, \vartheta 12, \vartheta 21, \vartheta 22, r]$;

$\mathrm{q}_{\mathrm{k}_{-}, \lambda_{-}}=$

$\left((-1) \wedge \lambda \omega /\left(r_{k, \lambda} \wedge 3 \epsilon\right)+2 /\left(r_{k, \lambda}{ }^{\wedge}-\kappa_{1} \wedge 2\right) \wedge 2+2 /\left(r_{k, \lambda} \wedge 2-\kappa_{2} \wedge 2\right) \wedge 2\right) \wedge(-1 / 2)$;

$\mathrm{u}_{\mathrm{s}_{-}, \lambda 1_{-}, \mathrm{k}_{-}, \lambda 2_{-}}=$

$\left((-1)^{\wedge}(\lambda 2-1) \operatorname{KroneckerDelta}[\lambda 1,1]-\right.$ in $\left.\operatorname{KroneckerDelta}[\lambda 1,2]\right) /$

$\left(2\left(r_{k, \lambda 2}-\kappa_{s}\right) \sqrt{r_{k, \lambda 2} \kappa_{s}}\right) q_{k, \lambda 2}$;

Q11 $=\mathrm{u}_{1,1,1,1} \mathrm{u}_{2,1,2,2}+\mathrm{u}_{2,1,1,1} \mathrm{u}_{1,1,2,2}$

$\theta 12=u_{1,1,1,1} u_{2,2,2,2}+u_{2,2,1,1} u_{1,1,2,2}$

$\theta 21=u_{1,2,1,1} u_{2,1,2,2}+u_{2,1,1,1} u_{1,2,2,2}$;

$\theta 22=u_{1,2,1,1} u_{2,2,2,2}+u_{2,2,1,1} u_{1,2,2,2}$;

$a=\left(1 / 4 \sqrt{\kappa_{2} r_{1,1}}\left(-\kappa_{2}+r_{1,1}\right) \sqrt{-\frac{\omega}{\epsilon r_{1,1}^{3}}+\frac{2}{\left(-\kappa_{1}^{2}+r_{1,1}^{2}\right)^{2}}+\frac{2}{\left(-\kappa_{2}^{2}+r_{1,1}^{2}\right)^{2}}}\right.$

$\left.\left.\sqrt{\kappa_{1} r_{2,2}}\left(-\kappa_{1}+r_{2,2}\right) \sqrt{\frac{\omega}{\epsilon r_{2,2}^{3}}+\frac{2}{\left(-\kappa_{1}^{2}+r_{2,2}^{2}\right)^{2}}+\frac{2}{\left(-\kappa_{2}^{2}+r_{2,2}^{2}\right)^{2}}}\right)\right) ;$

$\mathrm{b}=1 / 4 \sqrt{\kappa_{1} r_{1,1}}\left(-\kappa_{1}+r_{1,1}\right) \sqrt{-\frac{\omega}{\epsilon r_{1,1}^{3}}+\frac{2}{\left(-\kappa_{1}^{2}+r_{1,1}^{2}\right)^{2}}+\frac{2}{\left(-\kappa_{2}^{2}+r_{1,1}^{2}\right)^{2}}}$

$\left.\sqrt{\kappa_{2} r_{2,2}}\left(-\kappa_{2}+r_{2,2}\right) \sqrt{\frac{\omega}{\epsilon r_{2,2}^{3}}+\frac{2}{\left(-\kappa_{1}^{2}+r_{2,2}^{2}\right)^{2}}+\frac{2}{\left(-\kappa_{2}^{2}+r_{2,2}^{2}\right)^{2}}}\right) ;$

$y=4\left(a^{\wedge} 2-b^{\wedge} 2\right) ;$

$011=-a-b$

$\theta 12=$ ii $a-$ in $b$

$\theta 21=-\theta 12$

$\vartheta 22=\theta 11$

$-\left(1 / 4 \sqrt{\kappa_{2} r_{1,1}}\left(-\kappa_{2}+r_{1,1}\right) \sqrt{-\frac{\omega}{\epsilon r_{1,1}^{3}}+\frac{2}{\left(-\kappa_{1}^{2}+r_{1,1}^{2}\right)^{2}}+\frac{2}{\left(-\kappa_{2}^{2}+r_{1,1}^{2}\right)^{2}}}\right.$

$\left.\sqrt{\kappa_{1} r_{2,2}}\left(-\kappa_{1}+r_{2,2}\right) \sqrt{\left.\frac{\omega}{\epsilon r_{2,2}^{3}}+\frac{2}{\left(-\kappa_{1}^{2}+r_{2,2}^{2}\right)^{2}}+\frac{2}{\left(-\kappa_{2}^{2}+r_{2,2}^{2}\right)^{2}}\right)}\right)-$

$1 / 4 \sqrt{\kappa_{1} r_{1,1}}\left(-\kappa_{1}+r_{1,1}\right) \sqrt{-\frac{\omega}{\epsilon r_{1,1}^{3}}+\frac{2}{\left(-\kappa_{1}^{2}+r_{1,1}^{2}\right)^{2}}+\frac{2}{\left(-\kappa_{2}^{2}+r_{1,1}^{2}\right)^{2}}}$

$\left.\sqrt{k_{2} r_{2,2}}\left(-\kappa_{2}+r_{2,2}\right) \sqrt{\frac{\omega}{\epsilon r_{2,2}^{3}}+\frac{2}{\left(-\kappa_{1}^{2}+r_{2,2}^{2}\right)^{2}}+\frac{2}{\left(-\kappa_{2}^{2}+r_{2,2}^{2}\right)^{2}}}\right)$

True

True 
True

True

$r_{1,1}=\kappa_{1}+\epsilon \kappa_{1,1}$

$r_{2,2}=\kappa_{2}+\epsilon \kappa_{2,2}$

$\mathrm{y} 1=\operatorname{Normal}\left[\right.$ Assuming $\left.\left[\kappa_{1}<\kappa_{2}, \operatorname{Series}[\mathrm{y},\{\epsilon, 0,1\}]\right]\right]$

$-1+\epsilon\left(-\frac{2 \omega \kappa_{1,1}^{2}}{k_{1}}+\frac{2 \omega k_{2,2}^{2}}{k_{2}}\right)$

$\kappa_{1,1}=1 /\left(2\left(\kappa_{1}+\omega\right)\right) ;$

$\kappa_{2,2}=1 /\left(2\left(\kappa_{2}-\omega\right)\right)$;

$\left(-\frac{2 \omega \kappa_{1,1}^{2}}{\kappa_{1}}+\frac{2 \omega \kappa_{2,2}^{2}}{\kappa_{2}}\right) / /$ Simplify // ExpandNumerator

$\frac{1}{2}\left(-\frac{\omega}{\kappa_{1}\left(\omega+\kappa_{1}\right)^{2}}+\frac{\omega}{\left(\omega-\kappa_{2}\right)^{2} \kappa_{2}}\right)$ 


\section{B.3 Caso de três fótons no Mathematica}

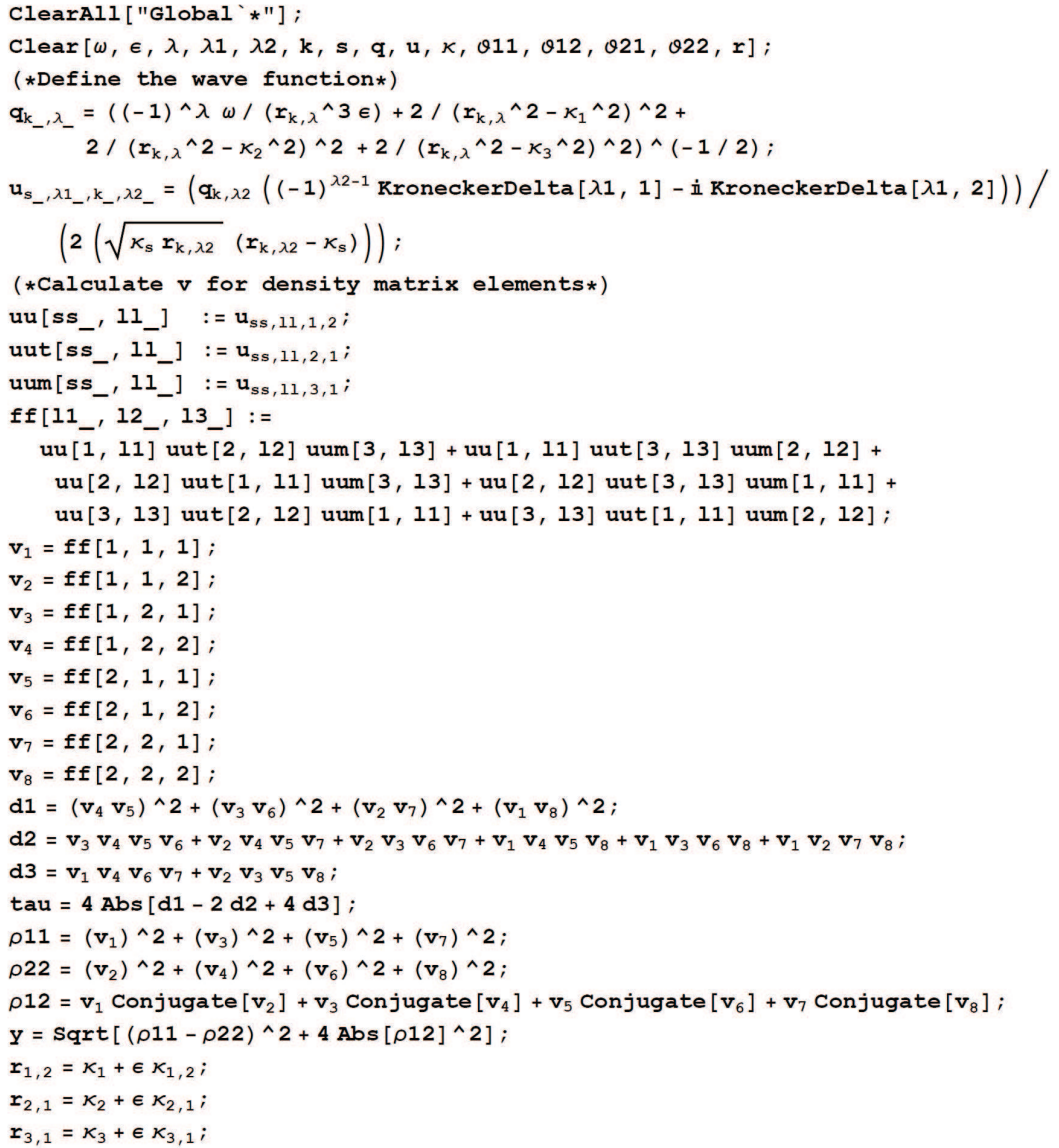


fterm $=$

Normal [Assuming $\left[\kappa_{1}>\kappa_{2}>\kappa_{3}\right.$, Series $[\operatorname{uu}[1,1]$ uut $[2,1]$ uum $\left.\left.[3,1],\{\epsilon, 0,1\}]\right]\right]$; $\mathrm{v} 1 \mathrm{e}=\operatorname{Normal}\left[\right.$ Assuming $\left.\left[\kappa_{1}>\kappa_{2}>\kappa_{3}, \operatorname{Series}\left[\mathrm{v}_{1},\{\epsilon, 0,1\}\right]\right]\right]$;

$\mathrm{v} 2 \mathrm{e}=\operatorname{Normal}\left[\right.$ Assuming $\left[\kappa_{1}>\kappa_{2}>\kappa_{3}\right.$, Series $\left.\left.\left[\mathrm{v}_{2},\{\epsilon, 0,1\}\right]\right]\right] ;$

$v 3 e=\operatorname{Normal}\left[\right.$ Assuming $\left.\left[\kappa_{1}>\kappa_{2}>\kappa_{3}, \operatorname{Series}\left[v_{3},\{\epsilon, 0,1\}\right]\right]\right] ;$

$\mathrm{v} 4 \mathrm{e}=\operatorname{Normal}\left[\right.$ Assuming $\left.\left[\kappa_{1}>\kappa_{2}>\kappa_{3}, \operatorname{Series}\left[\mathrm{v}_{4},\{\epsilon, 0,1\}\right]\right]\right] ;$

$\mathrm{v} 5 \mathrm{e}=\operatorname{Normal}\left[\right.$ Assuming $\left[\kappa_{1}>\kappa_{2}>\kappa_{3}\right.$, Series $\left.\left.\left[v_{5},\{\epsilon, 0,1\}\right]\right]\right] ;$

$\mathrm{v} 6 \mathrm{e}=\operatorname{Normal}\left[\right.$ Assuming $\left.\left[\kappa_{1}>\kappa_{2}>\kappa_{3}, \operatorname{Series}\left[\mathrm{v}_{6},\{\epsilon, 0,1\}\right]\right]\right]$;

$\mathrm{v} 7 \mathrm{e}=\operatorname{Normal}\left[\right.$ Assuming $\left.\left[\kappa_{1}>\kappa_{2}>\kappa_{3}, \operatorname{Series}\left[\mathrm{v}_{7},\{\epsilon, 0,1\}\right]\right]\right] ;$

$\mathrm{v} 8 \mathrm{e}=\operatorname{Normal}\left[\right.$ Assuming $\left.\left[\kappa_{1}>\kappa_{2}>\kappa_{3}, \operatorname{Series}\left[\mathrm{v}_{8},\{\epsilon, 0,1\}\right]\right]\right] ;$

$a=1 /\left(2 \sqrt{2} \sqrt{\kappa_{1}^{2}} \sqrt{\kappa_{2}^{2}} \sqrt{\kappa_{3}^{2}} \sqrt{\frac{1}{\kappa_{1}^{2} \kappa_{1,2}^{2}}} \kappa_{1,2} \sqrt{\frac{1}{\kappa_{2}^{2} \kappa_{2,1}^{2}}} \kappa_{2,1} \sqrt{\frac{1}{\kappa_{3}^{2} \kappa_{3,1}^{2}}} \kappa_{3,1}\right) ;$

$\mathrm{b}=\left(\epsilon\left(-\omega \kappa_{2} \kappa_{3} \kappa_{1,2}^{2}+\omega \kappa_{1} \kappa_{3} \kappa_{2,1}^{2}+\omega \kappa_{1} \kappa_{2} \kappa_{3,1}^{2}\right)\right) /$

$\left(2 \sqrt{2} \kappa_{1} \sqrt{\kappa_{1}^{2}} \kappa_{2} \sqrt{\kappa_{2}^{2}} \kappa_{3} \sqrt{\kappa_{3}^{2}} \sqrt{\frac{1}{\kappa_{1}^{2} \kappa_{1,2}^{2}}} \kappa_{1,2} \sqrt{\frac{1}{\kappa_{2}^{2} \kappa_{2,1}^{2}}} \kappa_{2,1} \sqrt{\frac{1}{\kappa_{3}^{2} \kappa_{3,1}^{2}}} \kappa_{3,1}\right) ;$

$v 1 e=-a-b$

$\mathrm{v} 2 \mathrm{e}=\mathbf{i} \mathrm{a}+\dot{\mathrm{i}} \mathrm{b}$

$v 3 e==\dot{i} a+i b$

$v 4 e=a+b$

$v 5 e=-$ i $a-i b$

$\mathrm{v} 6 \mathrm{e}=\mathrm{-}-\mathrm{a}-\mathrm{b}$

$v 7 e=-a-b$

v8e $=$ in $a+$ in $b$

True

True

True

True

True

True

True

True

$\kappa_{2,1}=\frac{1}{2 \kappa_{1}-2 \omega} ;$

$\kappa_{1,2}=\frac{1}{2 \kappa_{2}+2 \omega}$;

$\kappa_{3,1}=\frac{1}{2 \kappa_{3}+2 \omega}$;

$\mathrm{b}=\frac{\dot{\mathrm{i}} \epsilon\left(-\omega \kappa_{2} \kappa_{3} \kappa_{1,2}^{2}+\omega \kappa_{1} \kappa_{3} \kappa_{2,1}^{2}+\omega \kappa_{1} \kappa_{2} \kappa_{3,1}^{2}\right)}{2 \sqrt{2} \kappa_{1} \sqrt{\kappa_{1}^{2}} \kappa_{2} \sqrt{\kappa_{2}^{2}} \kappa_{3} \sqrt{\kappa_{3}^{2}} \sqrt{\frac{1}{\kappa_{1}^{2} \kappa_{1,2}^{2}}} \kappa_{1,2} \sqrt{\frac{1}{\kappa_{2}^{2} \kappa_{2,1}^{2}}} \kappa_{2,1} \sqrt{\frac{1}{\kappa_{3}^{2} \kappa_{3,1}^{2}}} \kappa_{3,1}} ;$ 
Assuming $\left[\kappa_{1} \in \mathbb{R} \& \& \kappa_{2} \in \mathbb{R} \& \& \kappa_{3} \in \mathbb{R}\right.$, Simplify $\left.[\mathrm{b}]\right] / /$ Simplify

$\frac{i \in \omega\left(-2 \omega+2 \kappa_{1}\right)\left(\omega+\kappa_{2}\right)\left(\omega+\kappa_{3}\right)\left(-\frac{\kappa_{2} \kappa_{3}}{4\left(\omega+\kappa_{2}\right)^{2}}+\kappa_{1}\left(\frac{\kappa_{3}}{\left(2 \omega-2 \kappa_{1}\right)^{2}}+\frac{\kappa_{2}}{4\left(\omega+\kappa_{3}\right)^{2}}\right)\right)}{4 \sqrt{2} \sqrt{\left(\omega-\kappa_{1}\right)^{2}} \kappa_{1} \kappa_{2} \sqrt{\left(\omega+\kappa_{2}\right)^{2}} \kappa_{3} \sqrt{\left(\omega+\kappa_{3}\right)^{2}}}$

ClearAll ["Global *"] ;

$\mathrm{v}_{2}=-\mathrm{i} \mathrm{v}_{1} ; \mathrm{v}_{3}=-\mathrm{i} \mathrm{v}_{1} ; \mathrm{v}_{4}=-\mathrm{v}_{1} ; \mathrm{v}_{5}=$ i $\mathrm{v}_{1} ; \mathrm{v}_{6}=\mathrm{v}_{1} ; \mathrm{v}_{7}=\mathrm{v}_{1} ; \mathrm{v}_{8}=-$ i $\mathrm{v}_{1}$

$\mathbf{y}=\sqrt{ }\left(\left(\operatorname{Abs}\left[\mathbf{v}_{1}\right]^{2}+\operatorname{Abs}\left[\mathbf{v}_{3}\right]^{2}+\operatorname{Abs}\left[\mathbf{v}_{5}\right]^{2}+\operatorname{Abs}\left[\mathbf{v}_{7}\right]^{2}-\operatorname{Abs}\left[\mathbf{v}_{2}\right]^{2}-\right.\right.$

$\left.\operatorname{Abs}\left[\mathrm{v}_{4}\right]^{2}-\mathrm{Abs}\left[\mathrm{V}_{6}\right]^{2}-\operatorname{Abs}\left[\mathrm{V}_{8}\right]^{2}\right)^{2}+4 \mathrm{Abs}\left[\left(\mathrm{v}_{1}\right.\right.$ Conjugate $\left[\mathrm{v}_{2}\right]+$

$v_{3}$ Conjugate $\left[v_{4}\right]+v_{5}$ Conjugate $\left[v_{6}\right]+v_{7}$ Conjugate $\left.\left.\left.\left[v_{8}\right]\right)\right] \wedge 2\right) ;$

Assuming $\left[\mathrm{v}_{1} \in \mathbb{R}\right.$, Simplify $\left.[\mathrm{y}]\right]$

$8 \mathrm{v}^{2}$

$\mathrm{d} 1=\left(\mathrm{v}_{4} \mathrm{v}_{5}\right) \wedge 2+\left(\mathrm{v}_{3} \mathrm{v}_{6}\right) \wedge 2+\left(\mathrm{v}_{2} \mathrm{v}_{7}\right) \wedge 2+\left(\mathrm{v}_{1} \mathrm{v}_{8}\right) \wedge 2 ;$

$\mathrm{d} 2=\mathrm{v}_{3} \mathrm{v}_{4} \mathrm{v}_{5} \mathrm{v}_{6}+\mathrm{v}_{2} \mathrm{v}_{4} \mathrm{v}_{5} \mathrm{v}_{7}+\mathrm{v}_{2} \mathrm{v}_{3} \mathrm{v}_{6} \mathrm{v}_{7}+\mathrm{v}_{1} \mathrm{v}_{4} \mathrm{v}_{5} \mathrm{v}_{8}+\mathrm{v}_{1} \mathrm{v}_{3} \mathrm{v}_{6} \mathrm{v}_{8}+\mathrm{v}_{1} \mathrm{v}_{2} \mathrm{v}_{7} \mathrm{v}_{8}$;

$\mathrm{d} 3=\mathrm{v}_{1} \mathrm{v}_{4} \mathrm{v}_{6} \mathrm{v}_{7}+\mathrm{v}_{2} \mathrm{v}_{3} \mathrm{v}_{5} \mathrm{v}_{8}$;

tau $=4$ Abs $[\mathrm{d} 1-2 \mathrm{~d} 2+4 \mathrm{~d} 3]$;

Assuming $\left[\mathrm{v}_{1} \in \mathbb{R}\right.$, Simplify $[$ tau $\left.]\right]$ 